\author{
Berichte aus dem \\ Institut für Systemdynamik \\ Universität Stuttgart
}

\title{
Steffen Kehl
}

\section{Querregelung eines Versuchsfahrzeugs entlang vorgegebener Bahnen}

D 93 (Diss. Universität Stuttgart)

Shaker Verlag

Aachen 2007 


\section{Bibliografische Information der Deutschen Nationalbibliothek}

Die Deutsche Nationalbibliothek verzeichnet diese Publikation in der Deutschen Nationalbibliografie; detaillierte bibliografische Daten sind im Internet über http://dnb.d-nb.de abrufbar.

Zugl.: Stuttgart, Univ., Diss., 2007

Copyright Shaker Verlag 2007

Alle Rechte, auch das des auszugsweisen Nachdruckes, der auszugsweisen odervollständigen Wiedergabe, der Speicherung in Datenverarbeitungsanlagen undder Übersetzung, vorbehalten.

Printedin Germany.

ISBN 978-3-8322-6542-7

ISSN 1863-9046

Shaker Verlag GmbH • Postfach $101818 \cdot 52018$ Aachen

Telefon: 02407 / 9596 - 0 - Telefax: 02407 / 9596 - 9

Internet:www.shaker.de•E-Mail:info@shaker.de 


\title{
Querregelung eines Versuchsfahrzeugs entlang vorgegebener Bahnen
}

\author{
Von der Fakultät Maschinenbau der Universität Stuttgart \\ zur Erlangung der Würde eines \\ Doktor-Ingenieurs (Dr.-Ing.) genehmigte Abhandlung
}

\author{
Vorgelegt von \\ Steffen Kehl \\ aus Stuttgart
}

Hauptberichter:

Prof. Dr.-Ing. Dr.h.c. Michael Zeitz

Mitberichter:

Prof. Dr.-Ing. habil. Jörg Wagner

Tag der mündlichen Prüfung: 15. Juni 2007

Institut für Systemdynamik

Universität Stuttgart 



\section{Vorwort}

Die vorliegende Arbeit entstand während meiner Tätigkeit von 2003 bis 2006 als Doktorand im Entwicklungszentrum der Dr. Ing. h.c. F. Porsche AG in Weissach.

Mein besonderer Dank für die ausgezeichnete Betreuung und Unterstützung dieser Arbeit gilt Herrn Prof. Dr.-Ing. Dr. h.c. Michael Zeitz vom Institut für Systemdynamik der Universität Stuttgart, dem Leiter der Abteilung Messtechnik der Porsche AG, Herrn Dipl.-Ing. Wolf-Dieter Pölsler und dem Leiter der Messtechnik-Fachabteilung Systemtechnik, Herrn Dipl.-Ing. Reinhold Bieg. Deren stetes Interesse, die vielfältigen Anregungen und die mir eingeräumten fachlichen Freiräume haben wesentlich zu dem Gelingen dieser Arbeit beigetragen. Ebenfalls herzlichen Dank an Herrn Prof. Dr.--Ing. habil. Jörg Wagner für seine konstruktiven Anmerkungen zu dem messtechnischen Teil der Arbeit und die freundliche Übernahme des Mitberichts. Mein Dank richtet sich auch an die Leiter des Instituts für Systemdynamik der Universität Stuttgart, Herrn Prof. Dr.-Ing. Oliver Sawodny sowie an Herrn Prof. Dr.-Ing. Dr. h.c. mult. Ernst Dieter Gilles, der das Institut bis 2005 geleitet hat.

Ich danke allen Kolleginnen und Kollegen der Abteilung Messtechnik für die gute Zusammenarbeit und das angenehme Arbeitsklima. Besonders danken möchte ich in diesem Zusammenhang Herrn Dipl.-Ing. Jochen Held für das sorgfältige Erarbeiten der ersten Rohdaten des Navigationssystems sowie die Vorbereitung der ersten geregelten Fahrversuche auf dem Prüfgelände. Einen besonderen Dank auch an Herrn Dipl.-Ing. Volker Stahl für die hervorragende Zusammenarbeit bei der Umsetzung der Navigations- und Regelalgorithmen auf die echtzeitfähige Hardware.

Ebenfalls danken möchte ich Herrn Dipl.-Ing. Benjamin Herzer, der durch sein Praktikum einen wertvollen Beitrag geleistet hat. 


\section{Inhaltsverzeichnis}

$\begin{array}{ll}\text { Kurzfassung } & \text { VII }\end{array}$

Abstract $\quad$ X

1 Einführung 1

1.1 Zielsetzung der Arbeit . . . . . . . . . . . . . . . 2

1.2 Umfeld der Arbeit . . . . . . . . . . . . . . . . . . 4

1.3 Gliederung der Arbeit . . . . . . . . . . . . . . 6

2 Trajektorienplanung für die Steuerung und Regelung 8

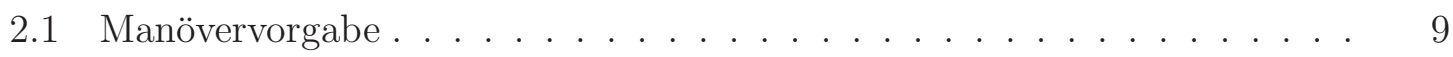

2.2 Sollbahnbestimmung . . . . . . . . . . . . . . . . . 11

2.3 Echtzeitberechnung der Solltrajektorie . . . . . . . . . . . . . 14

2.4 Neuplanung der Trajektorie bei großen Regelabweichungen . . . . . . . . 16

2.4.1 Splineplanung zu Beginn der Fahrt . . . . . . . . . . . . . 17

2.4.2 Splineplanung beim Überschreiten der Reifenhaftungsgrenze . . . 18

2.5 Zusammenfassung . . . . . . . . . . . . . . . . . . 20

3 Messung der Fahrzeugbewegung $\quad 22$

3.1 Inertiales Navigationssystem . . . . . . . . . . . . . . . . . 23

3.1.1 Koordinatensysteme ..................... 24

3.1.2 Strapdown-Navigationsrechnung . . . . . . . . . . . . . . 27

3.1 .3 Anfangsausrichtung . . . . . . . . . . . . . . . . . 29

3.2 Integriertes Navigationssystem . . . . . . . . . . . . . . . . 30

3.2.1 Fehlermodell der Strapdown-Rechnung . . . . . . . . . . . . 31

3.2.2 Beobachtbarkeit des Fehlerzustandes . . . . . . . . . . . 33

3.2.3 Stützung der Navigationsrechnung mittels Differential-GPS . . . 34

3.2.4 Synchronisierung der Sensoren . . . . . . . . . . . . . . . . . 36 
3.2 .5 Erweitertes Kalman-Filter . . . . . . . . . . . . . . . . . . . 36

3.3 Berechnung fahrdynamikrelevanter Größen . . . . . . . . . . . . . . . . . 40

3.4 Zusammenfassung . . . . . . . . . . . . . . . . . . . . . . . . . . 41

4 Querregelung für die Bahnfolge eines Versuchsfahrzeugs 43

4.1 Fahrzeugmodellierung . . . . . . . . . . . . . . . . . . . . . . . . 43

4.1.1 Kinematisches Fahrzeugmodell . . . . . . . . . . . . . . . 44

4.1 .2 Dynamisches Fahrzeugmodell . . . . . . . . . . . . . . . . . 46

4.1 .3 Reifenkennlinien für stationäre Fahrt . . . . . . . . . . . . . . . . 49

4.2 Regelungsstruktur mit zwei Freiheitsgraden . . . . . . . . . . . . . . . . 50

4.2 .1 Folgeregelung für langsame Fahrt . . . . . . . . . . . . . . . . 51

4.2 .2 Folgeregelung für schnelle Fahrt . . . . . . . . . . . . . . . . . 52

4.2.3 Folgeregelung für alle Geschwindigkeiten . . . . . . . . . . . . 57

4.3 Simulation der Querregelung . . . . . . . . . . . . . . . . . 58

4.4 Zusammenfassung . . . . . . . . . . . . . . . . . . . . 60

5 Implementierung und Erprobung der Bahnfolgeregelung 62

5.1 Implementierung des Gesamtsystems . . . . . . . . . . . . . . . . . . . 62

5.1 .1 Integriertes Navigationssystem . . . . . . . . . . . . . 65

5.1 .2 Trajektorienplanung und Querregelung . . . . . . . . . 66

5.1 .3 Lenkmaschine . . . . . . . . . . . . . . . . . . . . 67

5.1.4 Benutzerschnittstelle und Datenaufzeichnung . . . . . . . . . 68

5.2 Versuchsergebnisse . . . . . . . . . . . . . . . . . 70

5.2 .1 Doppelspurwechsel . . . . . . . . . . . . . . . 70

5.2.2 Versuchsfahrzeug Porsche Carrera . . . . . . . . . . . . 71

5.2.3 Versuchsergebnisse Porsche Carrera . . . . . . . . . . . . 73

5.2.4 Versuchsergebnisse Porsche Cayenne . . . . . . . . . . . . 78

5.3 Zusammenfassung . . . . . . . . . . . . . . . . . . . . . 80

$\begin{array}{llr}6 & \text { Schlußfolgerungen } & 81\end{array}$

Anhang: Die Dynamikmatrix des Fehlermodells $\quad 84$

$\begin{array}{ll}\text { Literaturverzeichnis } & 85\end{array}$ 


\section{Kurzfassung}

Um die fahrdynamischen Eigenschaften eines in der Entwicklung befindlichen Personenkraftwagens zu dokumentieren und zu verbessern, werden Fahrversuche auf dazu geeigneten Prüfgeländen definiert und durchgeführt. Der Grund für die Durchführung von Fahrversuchen ist die Gewinnung von objektiven und damit vergleichbaren Kenngrößen des untersuchten Fahrzeugs durch Auswertung der aufgezeichneten Messgrößen. Um die erforderliche Reproduzierbarkeit sicherzustellen, wird bei einem Teil der Versuche der Lenkwinkel in Form einer Steuerung durch eine elektrische Lenkmaschine vorgegeben. Ein weiterer Teil der Versuche erfordert darüber hinaus die Führung der Fahrzeugposition entlang einer Sollbahn. Die Bahnfolgeregelung konnte bisher nur durch den Versuchsingenieur realisiert werden. Die noch fehlende Automatisierung dieser Versuche ist hauptsächlich dadurch begründet, daß eine Regelung die Dynamik des Fahrzeugs und der Reifen für die Durchführung von aggressiven Manövern berücksichtigen muß. Ein weiterer Kernpunkt ist die Datenerfassung und die Realisierung des Regelsystems in Echtzeit. Aufgrund dieser Aufgaben ist das Ziel der vorliegenden Arbeit die Entwicklung einer Bahnfolgeregelung, die den Fahrzeugschwerpunkt über die Lenkung des Fahrzeugs entlang einer vorgegebenen Bahn führt.

Die Folgeregelung verwendet eine Lenkmaschine als Stellglied und ein integriertes Navigationssystem für die Messung. Kupplung, Schaltung, Gas und Bremse werden weiter durch den Versuchsingenieur bedient. Somit kann die Folgeregelung keinen Einfluß auf die Geschwindigkeit des Fahrzeugs nehmen und wird daher in Form einer Querregelung realisiert. Die Bahnfolgeregelung soll einerseits Manöver mit Querbeschleunigungen des Fahrzeugs an der Reifenhaftgrenze beherrschen und mit aggressiven Richtungsänderungen zurechtkommen, andererseits auch problemlos aus dem Stillstand heraus funktionieren.

Die von dem Regler benötigte Sollbahnkrümmung wird in Form der Koeffizienten eines stückweise definierten Polynomvektors hinterlegt. Der Grad der Polynome ist so gewählt, daß bei Verfolgung dieser Sollbahn durch das Fahrzeug keine Sprünge in der Lenkwinkelgeschwindigkeit vorkommen. Wenn auch die kinematischen Grenzen und die Grenzen der Reifenhaftung beachtet werden, ist diese Sollbahn realisierbar. Der Polynomparameter, der die Sollposition des Fahrzeugs auf der Sollbahn kennzeichnet, wird während der Fahrt so nachgeführt, daß die Sollposition stets der Punkt auf der Sollbahn ist, welcher der Istposition des Fahrzeugschwerpunktes geometisch am nächsten ist. 
Zur Messung der tatsächlichen Position und Bewegung des Fahrzeugs dient ein integriertes Navigationssystem. Das Navigationssystem ist so aufgebaut, daß das Ergebnis in Echtzeit zur Verfügung steht und für die Bahnfolgeregelung verwendbar ist. Die Messwerte des Navigationssystems dienen aber nicht nur der Bahnfolgeregelung. Sie werden während der Fahrt aufgezeichnet und ermöglichen eine spätere Auswertung des Fahrverhaltens zur Ermittlung der gewünschten objektiven Fahrzeugkenngrößen.

Die Bahnfolgeregelung wird in Form einer Zwei-Freiheitsgrad-Struktur aus nichtlinearer Vorsteuerung und linearer Regelung realisiert, um einen unabhängigen Entwurf des Führungs- und Störverhaltens zu ermöglichen. Für den modellbasierten Entwurf der Bahnfolgeregelung wird das Einspurmodell verwendet. Bei diesem Fahrzeugmodell werden die Vorder- und Hinterreifen zu jeweils einem Reifen zusammengefasst. Dadurch wird das Modell sehr übersichtlich, verbunden mit dem Nachteil, daß die reale Radlastverschiebung in Richtung der kurvenäußeren Räder vernachlässigt wird. Diese Radlastverschiebung hat im oberen Querbeschleunigungsbereich großen Einfluß auf das Fahrverhalten. Um diesen Nachteil auszugleichen, werden für das Einspurmodell nicht die nominellen Reifenkennlinien verwendet. Die Reifenkennlinien werden aus dem simulierten Verhalten des Fahrzeugs bei stationärer Kreisfahrt extrahiert. Als Resultat wird so der Einfluß der Schwerpunktlage und der sehr komplexen Fahrwerkskinematik in die Reifenkennlinien übernommen.

Die Vorsteuerung basiert auf der Kräftebilanz des nichtlinearen Einspurmodells in der Ebene. Die für die gewünschte Bahnkrümmung an den Vorderrädern benötigte Seitenkraft wird durch die modellbasierte Vorsteuerung und die lineare Regelung bestimmt. In einem weiteren Schritt wird die ermittelte Seitenkraft anhand des messbaren Fahrzeugzustandes und der bekannten Reifenkennlinien in einen Lenkradwinkel transformiert und eingestellt.

Die entworfene Bahnfolgeregelung wird mithilfe eines detaillierten und validierten Fahrzeugmodells unter MatLab/Simulink simuliert und überprüft. Als Testmanöver dient neben anderen Sollbahnen ein doppelter Fahrspurwechsel („Elchtest“), welcher sich durch Querbeschleunigungen bis an die Reifenhaftgrenze und schnelle Richtungswechsel mit damit verbundenen Radlaständerungen auszeichnet.

Nach Absicherung der korrekten Funktion durch die Simulation wurde die Bahnfolgeregelung als echtzeitfähiges System mit Bedienerschnittstelle an realen Fahrzeugen vom Typ Porsche Carrera und Porsche Cayenne appliziert. Die Fahrversuche wurden auf dem Prüfgelände der Porsche $A G$ in Weissach durchgeführt.

Das Verhalten der Bahnfolgeregelung in der Praxiserprobung entspricht den Ergebnissen der Simulationsuntersuchung. Als Beispielmanöver wird wie in der Simulation ein Doppelspurwechsel gewählt. Die größte Abweichung des Fahrzeugschwerpunktes von der Sollbahn liegt im Bereich der Reifenbreite, obwohl die Querbeschleunigung die physikalische Grenze erreicht oder sogar kurzfristig überschreitet. 
Durch die Bahnfolgeregelung wird eine Unabhängigkeit des Lenkwinkels vom Fahrer erreicht, die es beispielsweise erlaubt, verschiedene Fahrzeuge anhand des jeweiligen zur Verfolgung der Sollbahn notwendigen Lenkwinkelverlaufes untereinander zu vergleichen. Mit der Verfügbarkeit einer automatischen Bahnfolgeregelung wird die Zahl maschinell durchführbarer Fahrdynamikversuche zur Verbesserung der Reproduzierbarkeit erweitert und die Definition neuer Fahrversuche zur objektiven Gewinnung von Fahrzeugkenngrößen ermöglicht. 


\section{Abstract}

Driving tests for passenger cars are defined and conducted on suitable test tracks to document and to improve the dynamic characteristics of a vehicle in the course of its development. The motivation for conducting driving tests is to obtain objective and comparable information of the driving performance of the tested cars after the evaluation of the recorded measurement data. A part of the test drives are performed by using desired time profiles of the steering wheel angle, which are realised by an electrical steering machine in order to assure the necessary reproducibility. A further important class of test drives concerns the lateral control of the vehicle position along a predefined path. This path-following control can, up to now, only be realized by a test engineer. The main reasons for the present absence of automation in these path-following test scenarios is due to the fact that a controller has to account for the car dynamics and tire characteristics in order to realize aggressive maneuvers for meaningful test scenarios. A further issue is the acquisition of measurement data and the realization of the control concept in realtime. Motivated by these problems, the topic of this thesis is to develop an automated path-following control in order to guide the vehicle's centre of gravity along a predefined path by solely manipulating the steering angle of the vehicle.

The implementation of the path-following control uses a steering machine as actuator and an integrated navigation system as measurement device. Since the control scheme only manipulates the steering angle, the clutch, gearbox, throttle, and brakes have to be actuated by the test engineer. Therefore, the path-following control system has no direct effect on the vehicle's velocity and has to be realized as a lateral control concept. To conduct aggressive maneuvers and driving tests, the path-following control system must be able to handle lateral accelerations of the vehicle close to the physical friction limits of the tires. If the vehicle is in standstill, e.g. at the beginning of a test drive, the path-following control system has to automatically guide the car to the predefined path if the driver accelerates the car.

The curvature of the predefined path required by the controller is described in terms of the coefficients of a piecewise defined polynomial vector. The polynomial degree is chosen in order to avoid discontinuities in the steering wheel angle velocity when following the predefined path. The feasibility of the predefined path is ensured by additionally accounting for the kinematic constraints and the maximum available tire friction. The 
desired vehicle position on the predefined path is described by a polynomial parameter, which is dynamically updated such that the desired car position is the momentarily closest point on the path with respect to the actual position of the vehicle's centre of gravity.

An integrated navigation system measures the actual position and movement of the vehicle in real-time and transmits the measurement data to the path-following control system. Moreover, the measurement data are recorded during the driving maneuver to subsequently analyse and evaluate the vehicle's dynamic behaviour.

The path-following control is based on a two-degree of freedom structure with a nonlinear feedforward and a linear feedback control in order to separately design the reference action and disturbance action of the control loop. A single track model is used for the modelbased design of the path-following control. This model describes the vehicle dynamics by a simplified bicycle model with one tire at the front and rear of the vehicle. Thus the model becomes very concise at the price that the effect of load increase at the wheels on the outer-curve side is neglected. However, this load shift between the tire sides has a great influence on the behaviour of the vehicle at high lateral accelerations. To compensate for this drawback, the nominal tire characteristics are adapted in simulation studies to steady-state circular driving conditions. In this way, the influence of the position of the centre of gravity and the highly complex chassis kinematics are taken into account by the tire characteristics.

The feedforward control design is based on the balance of forces of the single track vehicle model on a plain surface. The desired curvature of the path corresponds to a required sideforce at the front wheels, which is determined by the model-based feedforward and the linear feedback control and is adjusted depending on the measurable vehicle state and the previously determined tire characteristics for the front and rear wheel of the vehicle model.

The path-following design is simulated and verified with a detailed and validated vehicle model by means of MatLab/Simulink. The test maneuver is a double lane change, although other predefined paths have been investigated as well. The double lane change is characterized by lateral acceleration at the physical limit of the tire friction and by fast directional changes with significant tire load changes.

After verifying the functionality in simulation studies, the path-following control has been implemented on a real-time-capable system with user interface, which has been installed in the passenger cars Porsche Carrera and Porsche Cayenne. The drive tests were conducted on the test track of Porsche AG in Weissach, Germany.

The performance of the realized path-following control for the chosen double lane change scenario corresponds to the simulation results. The largest deviation of the vehicle's centre of gravity from the desired path lies in the region of the tire width, although the lateral acceleration reaches the physical limits or even exceeds them momentarily. 
The realized path-following control allows reproducible test scenarios independent of the driver's driving abilities. This permits, for instance, the comparison of different vehicles for the same desired path by the necessary steering wheel activity. Moreover, the path-following control scheme leads to a higher reproducibility of the driving tests and allows the definition of novel test scenarios for the objective determination of vehicle characteristics. 


\section{Kapitel 1}

\section{Einführung}

Während der Entwicklung eines Personenkraftwagens werden im Bereich der Fahrdynamik verschiedene Fahrversuche definiert und durchgeführt. Diese Versuche dienen der Ermittlung des Fahrverhaltens zum Beispiel nach Modifikationen des Fahrwerks oder zum Vergleich verschiedener Fahrzeuge untereinander. Für solche Fahrversuche wird das Fahrzeug heutzutage gerne mit einem satellitengestützten inertialen Navigationssystem ausgerüstet. Dieses Navigationssystem misst mit hoher Präzision die Koordinaten der sechs Freiheitsgrade der Fahrzeugkarosserie. Im Anschluß an die Versuche werden die aufgezeichneten Messwerte analysiert und ausgewertet, um die gewünschten fahrdynamischen Größen zu ermitteln und eine Aussage über das Fahrzeugverhalten zu ermöglichen.

Zur Duchführung der Versuche müssen Lenkung, Gaspedal, Bremse, Kupplung und Getriebe des Fahrzeugs bedient werden. Diese Aufgabe wird in der Regel durch den Versuchsingenieur erfüllt. Für einen typischen Versuch wird das Fahrzeug aus dem Stand heraus auf eine bestimmte Geschwindigkeit beschleunigt, der Antriebsstrang in einen definierten Zustand gebracht und anschließend ein bestimmter Lenkwinkelverlauf realisiert [6].

Um die Versuche möglichst reproduzierbar und damit objektiv auswertbar durchzuführen, muß sich der Versuchsingenieur so gut wie möglich an die Manövervorgaben halten. Dabei können zum Beispiel mechanische Anschläge als Hilfsmittel zur Begrenzung des Lenkwinkels dienen. So können Lenkwinkelsprünge reproduzierbar durchgeführt werden. Werden komplexere Lenkwinkelverläufe benötigt, steigt der individuelle Einfluß des Fahrers auf den Versuch. Daher sollen die Versuche, die durch einen vorgeschriebenen zeitlichen Verlauf des Lenkwinkels charakterisiert sind, mit Hilfe einer Lenkmaschine realisiert werden.

Eine Lenkmaschine ist ein elektrischer Aktor, welcher anstelle des Lenkrades montiert wird. Die Lenkmaschine kann einen einmal programmierten Lenkwinkelverlauf in weiten Grenzen unahängig vom Lenkmoment beliebig oft reproduzieren. Dabei handelt es sich um eine reine Steuerung des Lenkwinkels. Die Reaktion des Fahrzeugs auf die Lenkwinkelveränderung wird für die Auswertung gemessen, hat aber keinen direkten Einfluß auf 


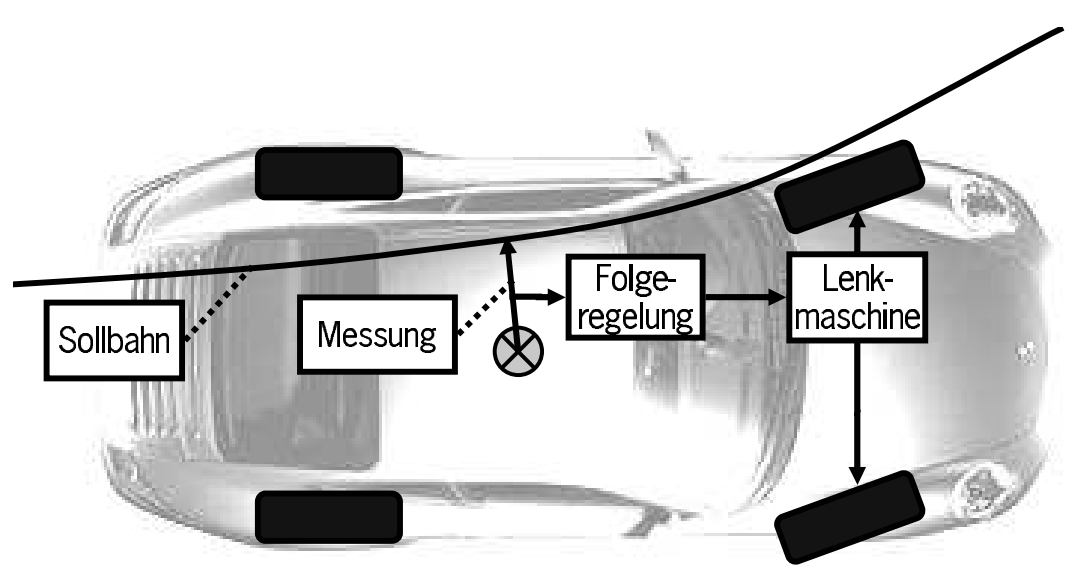

Abbildung 1.1: Prinzip der Bahnfolgeregelung für Fahrdynamikversuche

den Lenkwinkelverlauf.

Einige Manöver lassen sich nicht durch eine reine Steuerung der Lenkmaschine realisieren, da sie aufgrund der Versuchsdefinition das Nachfahren einer vorgegebenen Bahn erfordern. Beispiele dafür sind der als „Elchtest“ bekannt gewordene Doppelspurwechsel [13] oder eine Kreisfahrt [5]. Bei einem solchen Manöver hat der Versuchsingenieur die Aufgabe, das Fahrzeug bei einer gegebenen Geschwindigkeit entlang einer vorgegebenen Bahn zu führen. Er muß sich durch Probeläufe an das zunächst unbekannte Fahrzeug gewöhnen und entwickelt dabei eine bestimmte Fahrstrategie, um die Fahraufgabe unter den gegebenen Bedingungen zu bewältigen. Allerdings ist es einem menschlichen Fahrer kaum möglich, einer vorgegebenen Bahn mit einem gegebenen Punkt der Fahrzeugkarosserie zu folgen. In der Regel wird daher ein Korridor definiert, den das Fahrzeug während des Manövers nicht verlassen darf. Der Fahrer hat dann die Aufgabe, die Bahnplanung und Bahnfolge innerhalb dieses Korridors selbst durchzuführen. Dieser individuelle Einfluß des Versuchsingenieurs auf den Versuchsablauf ist abhängig von dessen Erfahrung, Können und Strategie und macht einen objektiven Vergleich von Messreihen schwierig oder verhindert ihn sogar [23].

\subsection{Zielsetzung der Arbeit}

Durch den Einsatz einer automatischen Querregelung des Versuchsfahrzeugs entlang vorgegebener Bahnen läßt sich die Abhängigkeit der Versuchsergebnisse von dem Versuchsingenieur weiter verringern. Durch die Unabhängigkeit des Lenkwinkels vom Fahrer für die Verfolgung einer bestimmten Bahn wird es möglich, verschiedene Fahrzeuge anhand des zur Verfolgung der Bahn notwendigen Lenkwinkelverlaufs zu vergleichen.

Dazu wird in dieser Arbeit eine Bahnfolgeregelung entwickelt, die den Fahrzeugschwerpunkt einer vorgegebenen Sollbahn mit möglichst geringem Abstand folgen läßt (Abbil- 


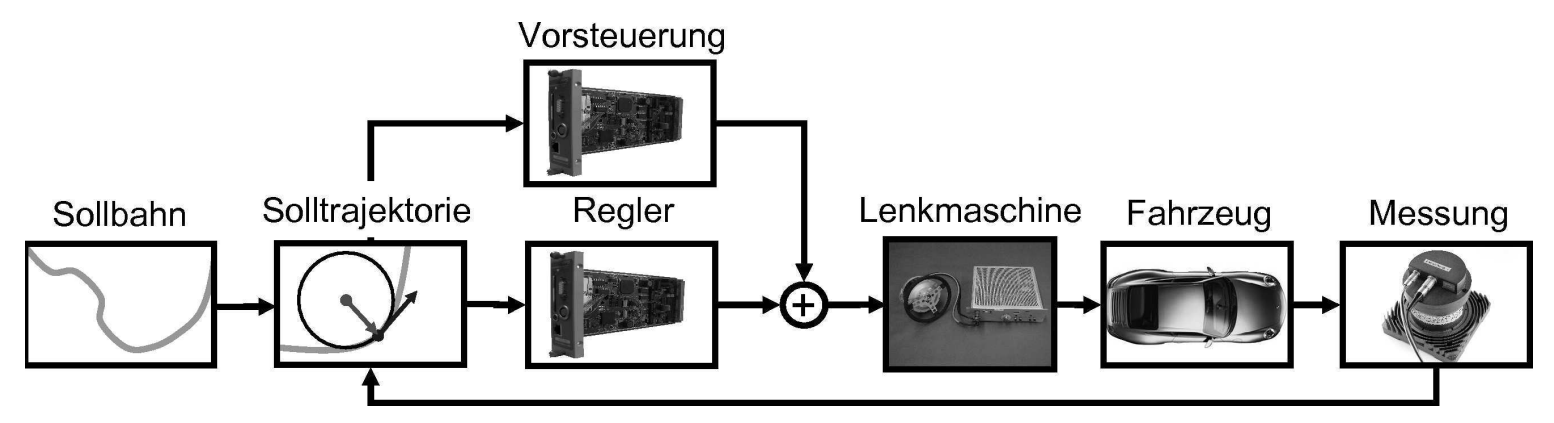

Abbildung 1.2: Struktur des realisierten Regelkreises

dung 1.1). Diese Bahnfolgeregelung soll für beliebige realisierbare Bahnen die Grenzen der Reifenhaftung ausschöpfen, aber auch beim Anfahren oder Anhalten bis zum Stillstand des Fahrzeugs funktionieren. Um das System flexibel an verschiedenen Orten einsetzbar zu machen, dürfen keine infrastrukturellen Maßnahmen nötig sein.

Als Aktor steht für die Bahnfolgeregelung die Lenkmaschine zur Verfügung, die ohnehin für die gesteuerten Manöver benötigt wird. Der Antriebsstrang muß bei der automatischen Bahnfolge wie bei den gesteuerten Manövern weiter durch den Versuchsingenieur bedient werden. Der Versuchsingenieur behält dadurch unter anderem die Aufgabe, im Störungsfall gegebenenfalls eine Notbremsung einzuleiten.

Die für die Versuchsauswertung benötigte Messtechnik kann auch für die Bahnfolgeregelung verwendet werden und stellt somit für die Realisierung der Bahnfolgeregelung keinen zusätzlichen Aufwand bei der Ausrüstung des Versuchsfahrzeugs dar. Sie umfaßt hier ein integriertes Navigationssystem, welches die Messung des Zustands der sechs Freiheitsgrade der Karosserie mit hoher Genauigkeit ermöglicht [7, 14]. Eine weitere Sensorik wird nicht benötigt.

Die Lenkmaschine, das Fahrzeug und die Messtechnik bilden einen offenen Regelkreis. Das Ziel dieser Arbeit ist, den Regelkreis zu schließen (Abbildung 1.2). Der Schwerpunkt liegt dabei auf der Bereitstellung einer Sollbahn, der Bestimmung einer Navigationslösung in Echtzeit sowie dem Entwurf und der Implementierung des Folgereglers. Als Rechnerplattform soll die für das Navigationssystem bereits bestehende Hardware verwendet werden, wodurch Aufwand und Kosten minimiert werden.

Neben der Lenkmaschine und der Messtechnik steht für die Fahrversuche mit Bahnfolgeregelung das Prüfgelände der Porsche $A G$ in Weissach mit seiner ebenen Fahrdynamikfläche zur Verfügung (Abbildung 1.3). Für die Versuche wird ein Sportwagen des Typs Porsche Carrera [26] und ein sportlicher Gelängewagen des Typs Porsche Cayenne [27] verwendet. Als wichtige Ressource für die simulationstechnische Erprobung der entworfenen Bahnfolgeregelung im Vorfeld der Praxisversuche existieren für diese Fahrzeuge detaillierte Fahrzeugmodelle, welche in MatLab/Simulink programmiert sind. Diese Modelle 


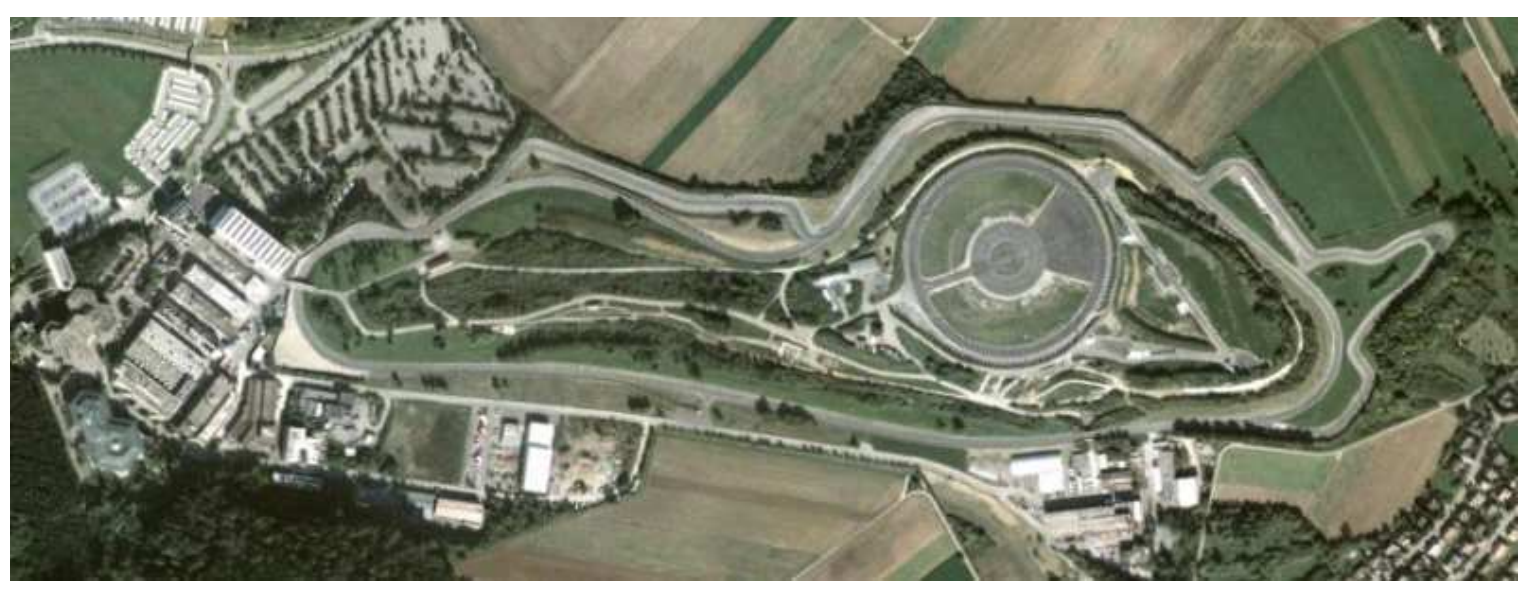

Abbildung 1.3: Das Entwicklungszentrum der Porsche AG in Weissach mit Prüfgelände und Fahrdynamikfläche (Bild: Google Earth)

wurden durch experimentelle Messungen validiert und bilden die realen fahrdynamischen Eigenschaften der Fahrzeuge sehr gut nach.

\section{$1.2 \quad$ Umfeld der Arbeit}

Im Bereich der Bahnfolgeregelung für Personenkraftwagen herkömmlicher Bauart ${ }^{1}$ gibt es zahlreiche Veröffentlichungen, die sich in die Bereiche autonomes Fahren, führerloses Fahren, Fahrassistenzsysteme und Fahrzeugregelung zum Zwecke der Fahrdynamikuntersuchung gruppieren lassen (s.u.). Einen Überblick über die Entwicklung automatisch geführter Fahrzeuge liefert [30].

Autonome Fahrzeuge Autonome Fahrzeuge müssen völlig ohne menschlichen Fahrer auskommen. Daher hat eine zuverlässige Pfadplanung bei autonomen Fahrzeugen eine sehr große Bedeutung. So sind beispielsweise militärische Einrichtungen sehr daran interessiert, unbemannte Fahrzeuge für besonders gefährliche Aufgaben in unbekanntem Gelände einzusetzten. Ein Beispiel dieses Interesses ist der Wettbewerb „Grand Challenge 2005“ der „Defense Advanced Research Projects Agency“ (DARPA). Die korrekte sensortechnische Erfassung und Verarbeitung der Umgebung ist hier besonders wichtig, um eine mögliche Fahrspur ermitteln zu können [33].

Führerlose Fahrzeuge Führerlose Fahrzeuge dienen dem Schienenersatz bei Transportsystemen. Ein Beispiel hierfür sind die Fahrzeuge für die Serviceröhre des Eurotunnels

\footnotetext{
${ }^{1}$ Damit ist ein PKW mit zwei gelenkten Vorderrädern und zwei ungelenkten Hinterrädern gemeint. Der Antrieb kann über die Vorder-, die Hinter- oder alle Räder erfolgen.
} 
oder fahrerlose Transportsysteme in der Automobilproduktion [9]. Bei diesen Systemen wird die Positionsbestimmung meist über einen in der Fahrbahn verlegten Leitdraht realisiert. Zugleich ist eine einmal festgelegte Bahn nur mit großem Aufwand veränderbar. Eine andere Möglichkeit der Positionsbestimmung besteht in Transpondern, die in regelmäßigen Abständen in der Fahrbahn versenkt werden. Dadurch können auch positionsabhängige Zusatzinformationen wie Bahnkrümmung und Sollgeschwindigkeit übertragen werden.

Leitdrahtgeführte Fahrzeuge dienen bei verschiedenen Automobilherstellern der Dauerfestigkeitserprobung. Die Fahraufgabe beschränkte sich hier jedoch bisher auf Fahrten mit hoher vertikaler Anregung auf Schlechtwegstrecken bei gleichzeitig geringen Querbeschleunigungen [25]. Der Nachteil leitdrahtgeführter Fahrzeuge liegt in der benötigten Infrastruktur und dem durch den Montageort des Sensors vorgegebenen Bezugspunkt für die Regelung. Sollte sich der Leitdraht außerhalb des Erfassungsbereichs des Sensors befinden, ist keine Bestimmung der Position und damit auch keine Regelung mehr möglich.

Fahrerassistenzsysteme Fahrerassistenzsysteme helfen dem Fahrer in Serienfahrzeugen, den Fahrwunsch auch unter schwierigen Bedingungen zu realisieren. Sie erleichtern zum Beispiel, Abstand zum vorausfahrenden Fahrzeug zu halten und im Notfall möglichst schnell abzubremsen, verhindern im Rahmen der physikalischen Grenzen ein ungewolltes Ausbrechen des Fahrzeugs und machen auf unbeabsichtigtes Verlassen der Spur aufmerksam [37]. Bei diesen Systemen stellt der menschliche Fahrer immer den übergeordneten Regler dar. Die Aufgabe von Fahrerassistenzsystemen besteht darin, den Wunsch des Fahrers zu erkennen und ihn bei der Realisierung dieses Wunsches zu unterstützen.

Bahnfolgeregelung zur Ermittlung objektiver Fahrzeugkenngrößen Im Gegensatz zu den beschriebenen Systemen dient eine Bahnfolgeregelung der Ermittlung objektiver Fahrzeugkenngrößen nicht der sicheren Erfüllung von Transportaufgaben. Ein solches System hilft den Entwicklern von Serien-Personenkraftwagen bei der Objektivierung von Fahrversuchen. Zur Untersuchung der Fahrdynamikeigenschaften werden neben gesteuerten Manövern auch Manöver durchgeführt, die das Nachfahren einer vorgegebenen Bahn und damit eine Bahnfolgeregelung erfordern. Dabei soll nach Möglichkeit der menschliche Fahrer durch ein Regelsystem ersetzt werden, um die Reproduzierbarkeit und den objektiven Vergleich zu verbessern. Diese Tests müssen flexibel auf den gerade zur Verfügung stehenden Flächen durchgeführt werden und können daher nicht auf fest installierte Infrastruktur (wie z.B. Leitdraht etc.) zur Bahnführung zurückgreifen [17, 15, 16, 18, 32]. 


\subsection{Gliederung der Arbeit}

Inhalt und Gliederung der Arbeit resultieren aus den in Abbildung 1.2 dargestellten Arbeitspaketen, die benötigt werden, um den Regelkreis zu schließen. Zunächst wird in Kapitel 2 auf die Bahn- und Trajektorienplanung für die Steuerung und Regelung eingegangen. Die Planung der Sollbahn wird nicht mehr während der Fahrt durch den Fahrer selbst innerhalb des vorgeschriebenen Korridors vorgenommen, sondern im Vorfeld der Versuche anhand des gegebenen Wunschmanövers. Daraus wird der Sollpositionsverlauf des Fahrzeugschwerpunktes als Ortskurve extrahiert. Um für das Fahrzeug realisierbar zu sein, muß die Sollbahn ausreichend glatt sein. Sie wird so für den Regler aufbereitet und hinterlegt, daß die Ortskurve während der Fahrt in Abhängigkeit von Fahrzeugposition und -geschwindigkeit in eine Solltrajektorie überführt werden und daraus die aktuelle Sollposition und -krümmung in Echtzeit berechnet werden kann.

Das zur Messung des Fahrzeugzustandes eingesetzte Navigationssystem wird in Kapitel 3 beschrieben. Dieses inertiale Navigationssystem wird zur Erreichung von Langzeitstabilität der Messung mithilfe von Differential-GPS über ein Kalman-Filter gestützt. Zur Verwendung im geschlossenen Regelkreis muß es echtzeitfähig sein und mit der zur Verfügung stehenden Rechenkapazität auskommen. Neben dem prinzipiellen Aufbau eines solchen Navigationssystems werden die praxisrelevanten Gesichtspunkte der Anfangsausrichtung, Beobachtbarkeit und Synchronisierung der Sensoren erläutert.

Mit der Bahnplanung und der Messtechnik ist die Basis für die in Kapitel 4 beschriebene Bahnfolgeregelung vorhanden. Für die Folgeregelung wird eine Regelungsstruktur mit zwei Freiheitsgraden, bestehend aus modellbasierter Vorsteuerung und stabilisierendem Regler, gewählt [10]. Für den Entwurf der Vorsteuerung werden in Abschnitt 4.1 sowohl ein kinematisches Einspurmodell des Fahrzeugs für langsame Fahrt als auch ein dynamisches Einspurmodell für schnelle Fahrt definiert. Der Abschnitt mit den Reifenkennlinien für stationäre Fahrt zeigt die Berücksichtigung des real über der Fahrbahnebene liegenden Schwerpunktes durch geeignete Wahl der Reifenkennlinien. Auf die Definition der Fahrzeugmodelle folgt in Abschnitt 4.2 der darauf basierende Entwurf der Vorsteuerung und Regelung. Hier wird wie bei der Modellbildung zwischen einer Folgeregelung für langsame Fahrt und einer Folgeregelung für schnelle Fahrt unterschieden. Ein validiertes und detailliertes Fahrzeugmodell auf Basis von MatLab/Simulink dient neben der Identifikation der Reifenkennlinien auch der simulationstechnischen Überprüfung der entworfenen Bahnfolgeregelung. Die Simulationsergebnisse werden in Abschnitt 4.3 am Beispiel eines doppelten Fahrspurwechsels vorgestellt.

In Kapitel 5 wird beschrieben, wie die echtzeitfähige Implementierung und die experimentelle Erprobung der Bahnfolgeregelung an Versuchsfahrzeugen vom Typ Porsche Carrera und Porsche Cayenne vorgenommen werden. Als Testmanöver wird mit dem doppelten Spurwechselmanöver dieselbe Sollbahn wie in der Simulation verwendet. Für dieses 
Manöver werden die Ergebnisse des Praxistests mit den beiden Fahrzeugen vorgestellt und mit dem in der Simulationsuntersuchung ermittelten Verhalten der Bahnfolgeregelung verglichen. 


\section{Kapitel 2}

\section{Trajektorienplanung für die Steuerung und Regelung}

Durch die Querregelung des Versuchsfahrzeugs soll die Ortskurve des Fahrzeugschwerpunktes unabhängig von der gefahrenen Geschwindigkeit mit der Ortskurve der Sollbahn übereinstimmen. Die in diesem Kapitel beschriebene Bahn- und Trajektorienplanung bildet die Basis für den Entwurf der Bahnfolgeregelung. Entsprechend des in Abbildung 2.1 gezeigten Schemas ist die Bahn- und Trajektorienplanung in eine Planungsphase (Abschnitt 2.1 und 2.2) und einen Berechnungsteil zur Bestimmung der Solltrajektorie in Echtzeit (Abschnitt 2.3) aufgeteilt.

Die Planungsphase betrifft die messtechnische oder auch synthetische Gewinnung einer Solltrajektorie aus einer Manövervorgabe. Die Manövervorgabe beeinhaltet eine Sollgeschwindigkeit des Fahrzeugs. Für die Solltrajektorie der Manövervorgabe ist zu beachten, daß das gewünschte Fahrmanöver als Trajektorie der Position des Fahrzeugschwerpunktes in der Ebene beschrieben wird. Dabei kann es sich zum Beispiel um eine Kreisfahrt [5], ein doppeltes Spurwechselmanöver [13] oder eine Fahrt entlang einer Dauerlaufstrecke [25] handeln.

Der Versuchsingenieur bedient im Fahrzeug Getriebe, Gaspedal und Bremse und beeinflusst damit die Geschwindigkeit entsprechend der Manövervorgabe. Die Querregelung des Versuchsfahrzeugs hat keinen direkten Einfluß auf die Fahrzeuggeschwindigkeit, da als einziger Aktor eine Lenkmaschine verwendet wird. Um die Sollbahn unabhängig von der Fahrzeuggeschwindigkeit vorzugeben, werden die aufgezeichneten bzw. vorgegebenen Punkte der zeitlichen Solltrajektorie durch eine geeignete Splinefunktion angenähert, um eine Darstellung der Manövervorgabe als örtliche Sollbahn zu erhalten. Bei der Bestimmung des Splines wird versucht, einen möglichst glatten und ruckarmen Verlauf der Sollbahn zu erzielen $[19,24]$. So wird erreicht, daß das Fahrzeug der Solltrajektorie folgen kann.

Vor Beginn der Versuchsfahrt werden die Polynomparameter der Splinesegmente der 


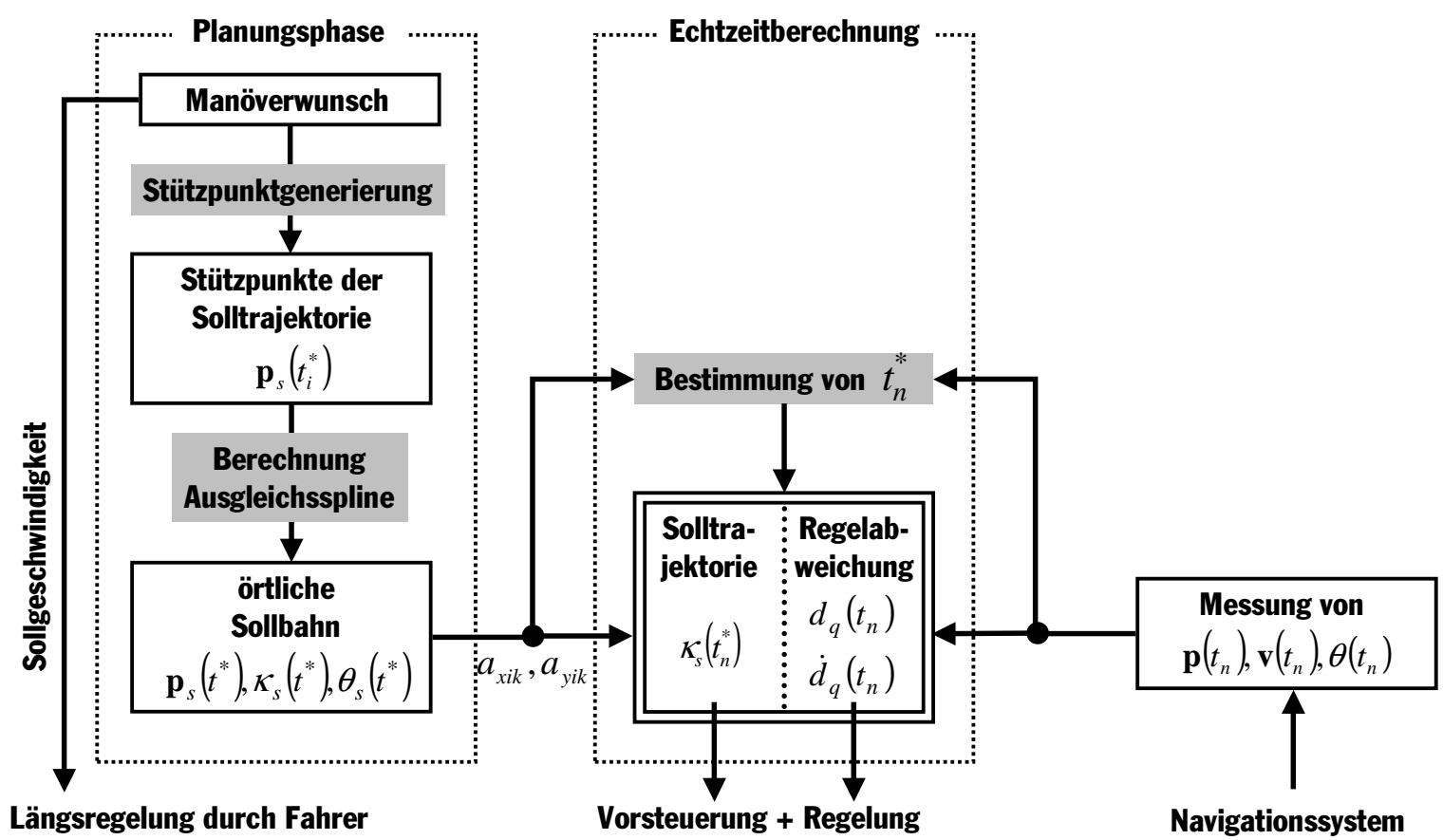

Abbildung 2.1: Planungs- und Berechnungsschema zur Bestimmung der Solltrajektorie und Regelabweichung aus der Manövervorgabe.

örtlichen Sollbahn auf einem Datenträger abgelegt. Mit den Polynomparametern wird während der Fahrt die Solltrajektorie in Abhängigkeit von der gemessenen Fahrzeugposition und -geschwindigkeit berechnet. Die in Echtzeit berechnete Solltrajektorie bildet die Basis für die Berechnung der Vorsteuerung in Abb. 1.2 mit Hilfe der Sollbahnkrümmung. Schließlich wird mit Hilfe des Sollbahnsplines die aktuelle Regelabweichung in Echtzeit berechnet. Die Regelabweichung ist die Distanz zwischen der Sollposition und der mit dem Navigationssystem gemessenen Position des Fahrzeugschwerpunkts (siehe Abb. 2.3).

\subsection{Manövervorgabe}

Ausgangspunkt der Planungsphase ist die einheitliche Beschreibung der einzelnen Fahrdynamikmanöver in einer Manövervorgabe. Sie beinhaltet die Information über das gewünschte Manöver und den zugehörigen Geschwindigkeitsverlauf des Fahrzeugs. Das durchzuführende Manöver liegt zunächst nicht in einer klar definierten Form vor. Die konkrete Manövervorgabe ergibt sich entweder aus existierenden Vorschriften für die Fahrdynamikerprobung, aus dem Wunsch nach reproduzierbar durchgeführten Fahrten zu Vergleichszwecken oder aus Anforderungen der Fahrzeugdauererprobung.

Das gewünschte Manöver muss für die Regelung in eine einheitliche Definition der Soll- 
trajektorie des Fahrzeugschwerpunktes übersetzt werden. Das Manöver ist zum Beispiel durch einen Korridor festgelegt, in dem sich das Fahrzeug bewegen darf und in welchen es mit einer bestimmten Anfangsgeschwindigkeit einfahren soll. In der bisherigen Vorgehensweise plant ein Versuchsingenieur eine geeignete Trajektorie innerhalb des vorgegebenen Korridors und versucht diese wiederholt und möglichst reproduzierbar durch manuelle Lenkung des Fahrzeugs zu verfolgen. Bei Einsatz der automatischen Bahnfolgeregelung wird hingegen einmalig eine Solltrajektorie innerhalb dieses Korridors als Manövervorgabe festgelegt, um diese dann beliebig oft automatisch abzufahren.

Eine anderes Wunschmanöver kann anstatt eines Korridors eine konkrete Solltrajektorie des Fahrzeugs beinhalten. Um das gewünsche Manöver in die einheitliche Form einer Manövervorgabe zu bringen, wird die Solltrajektorie in eine Folge von $N$ Stützpunkten $\mathbf{p}_{s}\left(t_{i}^{\star}\right)$ in der Nord-Ost-Ebene zu den Zeitpunkten $t_{i}^{\star}$ mit $i=1 \ldots N$ übertragen. Diese Stützpunkte enthalten durch ihren Orts- und Zeitbezug neben der Sollposition des Fahrzeugschwerpunktes auch dessen Sollgeschwindigkeit $\mathbf{v}_{s}\left(t_{i}^{\star}\right)$.

Es gibt die folgenden zwei Methoden, um die Stützpunkte der Solltrajektorie vorzugeben:

Messtechnische Vorgabe: Im einfachsten Fall werden das gewünschte Manöver durch den Versuchsingenieur „vorgefahren“ und die Stützpunkte $\mathbf{p}_{s}\left(t_{i}^{\star}\right)$ der sich ergebenden Trajektorie des Fahrzeugschwerpunktes mit Hilfe des integrierten Navigationssystems (siehe Kapitel 3) in einem festen Zeitraster $t_{i+1}^{\star}-t_{i}^{\star}=$ const aufgezeichnet. Somit ist die Realisierbarkeit der Trajektorie für das verwendete Fahrzeug unmittelbar sichergestellt. Der in drei Dimensionen gemessene Bahnverlauf wird in eine zur Erdoberfläche parallele Ebene projiziert und enthält dadurch keine Höheninformation mehr.

Synthetische Vorgabe: Die synthetische Vorgabe der Solltrajektorie erfolgt anhand des gewünschten Manövers. Aus dessen Beschreibung und den Versuchsanforderungen geht die Solltrajektorie für den Fahrzeugschwerpunkt hervor. Mit einem Hilfsprogramm wird die Solltrajektorie des Fahrzeugschwerpunktes manuell innerhalb der gegebenen Fahrbahngrenzen des Prüfgeländes definiert. Sie wird aus Bausteinen wie zum Beispiel Geraden, Kreissegmenten, Klothoiden ${ }^{1}$ und Splines zusammengesetzt. Aus der Solltrajektorie werden anschließend zeitlich äquidistante Stützpunkte entnommen und in die Ebene projiziert, so daß deren Stützpunkte $\mathbf{p}_{s}\left(t_{i}^{\star}\right)$ in derselben Form wie bei einer messtechnischen Vorgabe vorliegen.

Die zeitlich und örtlich punktuelle Definition der Solltrajektorie durch die Stützpunktgenerierung stellt eine Vorstufe in der Erzeugung der Sollbahn dar und erlaubt eine einheitliche Darstellung für die Sollbahnbestimmung in der Planungsphase (Abb. 2.1).

\footnotetext{
${ }^{1}$ Die Krümmung einer Klothoide oder Cornu-Spirale ist proportional ihrer Länge.
} 


\subsection{Sollbahnbestimmung}

Die Sollbahn muß so gestaltet sein, daß der Fahrzeugschwerpunkt ihr tatsächlich folgen kann. Die durch den Elektromotor der Lenkmaschine realisierbare Lenkwinkelgeschwindigkeit ist begrenzt und stetig. So kann die Lenkmaschine keine sprunghaften Winkeländerungen und das Fahrzeug daher keine plötzlichen Richtungswechsel durchführen. Um eine stetige Lenkwinkelgeschwindigkeit zu ermöglichen, muß die Beschleunigungsänderung (der Ruck) der Sollbahn stetig sein. Um die Anregung des Fahrzeugaufbaus $^{2}$ möglichst gering zu halten, soll zusätzlich zu der Stetigkeitsforderung der Ruck der Sollbahn minimiert werden.

Die Vorsteuerung und Regelung benötigen die Sollwerte von Position $\mathbf{p}_{s}\left(t^{\star}\right)$, Bahnkrümmung $\kappa_{s}\left(t^{\star}\right)$ und Fahrtrichtung $\theta_{s}\left(t^{\star}\right)$ für beliebige während der Fahrt in Echtzeit ermittelte Zeitpunkte $t^{\star}$ für $t_{1}^{\star} \leq t^{\star} \leq t_{N}^{\star}$. Für die Berechnung von Bahnkrümmung und -richtung werden Ableitungen der Position benötigt. Zu deren Berechnung in Echtzeit ohne Interpolation und numerische Differentiation und zur Erfüllung der Forderung nach einem stetigen Ruckverlauf werden die Stützpunkte $\mathbf{p}_{s}\left(t_{i}^{\star}\right)$ durch einen Ausgleichsspline 8. Ordnung [1] angenähert. Ausgleichssplines 8. Ordnung erfüllen die Forderung nach einem glattem Ruckverlauf durch ihre dreifach stetige Differenzierbarkeit. Die Anpassung des Ausgleichssplines an die Stützpunkte erfolgt so, daß bei minimalem Ruck der Abstand der Sollbahn zu den Stützpunkten akzeptabel klein bleibt [31]. Dadurch unterscheidet sich der verwendete Ausgleichsspline von Interpolationssplines, welche exakt durch die gegebenen Stützpunkte laufen. Durch die Minimierung des Rucks soll eine verhältnismäßig „sanfte“ Fahrt ohne ruckartige Lastwechsel und starke Fahrzeugaufbaubewegungen erreicht werden. Durch die gewählte Spline-Ordnung wird sichergestellt, daß die zur Verfolgung der Solltrajektorie erforderliche Lenkwinkelgeschwindigkeit mit der Lenkmaschine als stetiger Verlauf realisierbar ist.

Die Solltrajektorie ist in Form von $N$ Stützpunkten $\mathbf{p}_{s}\left(t_{i}^{\star}\right)=\left(x_{i}, y_{i}\right)$ für $N$ äquidistante Zeitpunkte $t_{i}^{\star}, i=1 \ldots N$ gegeben. Ein Spline besteht aus $N-1$ stückweise stetigen Spline-Segmenten

$$
s_{i}\left(t^{\star}\right)=\sum_{k=0}^{M-1} a_{i k}\left(t^{\star}-t_{i}^{\star}\right)^{k}, t^{\star} \in\left[t_{i}^{\star}, t_{i+1}^{\star}\right] .
$$

Die $M$ Parameter $a_{i k}, k=1, \ldots, M$ sind die Koeffizienten des $i$-ten Spline-Segments. Die Ableitungen der Splinesegmente (2.1) werden im Echtzeitteil der Sollbahnvorgabe zur Berechnung der Sollbewegungsrichtung und der Sollbahnkrümmung benötigt und

\footnotetext{
${ }^{2}$ Der Fahrzeugaufbau ist die gefederte Masse des Fahrzeugs
} 


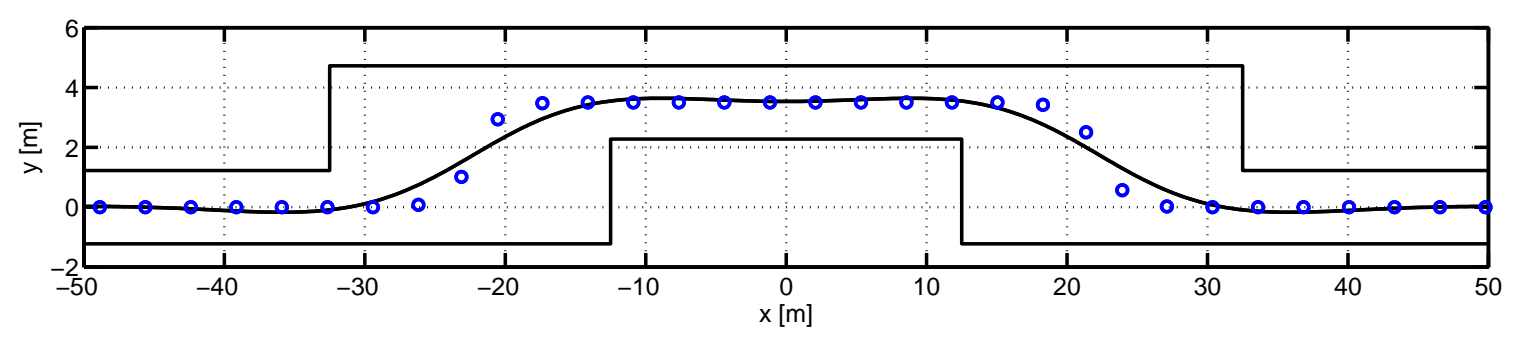

Abbildung 2.2: Korridor und Stützpunkte der Solltrajektorie eines Doppelspurwechsels und Approximation der Solltrajektorie für die Sollbahn durch einen Ausgleichsspline 8. Ordnung.

sind gegeben durch

$$
\begin{aligned}
s_{i}^{\prime}\left(t^{\star}\right) & =\sum_{k=1}^{M-1} k a_{i k}\left(t^{\star}-t_{i}^{\star}\right)^{k-1}, \\
s_{i}^{\prime \prime}\left(t^{\star}\right) & =\sum_{k=2}^{M-1} k(k-1) a_{i k}\left(t^{\star}-t_{i}^{\star}\right)^{k-2}, \\
s_{i}^{\prime \prime \prime}\left(t^{\star}\right) & =\sum_{k=3}^{M-1} k(k-1)(k-2) a_{i k}\left(t^{\star}-t_{i}^{\star}\right)^{k-3} .
\end{aligned}
$$

Für einen Spline in der Ebene, wie er für die Querregelung des Fahrzeugs benötigt wird, werden zwei Splines $s_{x}\left(t^{\star}\right)$ und $s_{y}\left(t^{\star}\right)$ der Form (2.1) zu einem Spline-Vektor $\mathbf{p}_{s}\left(t^{\star}\right)=$ $\left(s_{x}\left(t^{\star}\right), s_{y}\left(t^{\star}\right)\right)$ zusammengefaßt. Um einen Ausgleichsspline $\mathbf{p}_{s}\left(t^{\star}\right)$ zu finden, welcher in der Nähe der gegeben Stützpunkte $\left(x_{i}, y_{i}\right)$ verläuft und den Ruck minimiert, müssen die beiden Minimalitätskriterien Abstand und Ruck in beiden Achsen $x$ und $y$

$$
\begin{aligned}
& \min _{a_{x i k}}\left\{p_{x} \sum_{i=1}^{N}\left(s_{x}\left(t_{i}^{\star}\right)-x_{i}\right)^{2}+\int_{t_{1}}^{t_{N}}\left[s_{x}^{\prime \prime \prime}\left(t^{\star}\right)\right]^{2} \mathrm{~d} t^{\star}\right\} \\
& \min _{a_{y i k}}\left\{p_{y} \sum_{i=1}^{N}\left(s_{y}\left(t_{i}^{\star}\right)-y_{i}\right)^{2}+\int_{t_{1}}^{t_{N}}\left[s_{y}^{\prime \prime \prime}\left(t^{\star}\right)\right]^{2} \mathrm{~d} t^{\star}\right\}
\end{aligned}
$$

durch Optimierung der Splineparameter $a_{x i k}$ für $s_{x}\left(t^{\star}\right)$ und $a_{y i k}$ für $s_{y}\left(t^{\star}\right)$ erfüllt sein. Die Gewichtungsparameter $p_{x}=p$ und $p_{y}=p$ in (2.5) und (2.6) werden identisch gewählt und bestimmen die Verteilung der Priorität zwischen Abstandsminimierung zu den Stützpunkten und Minimierung des Rucks. Die Berechnung der Splineparameter erfolgt wie in [31] beschrieben durch analytische Bestimmung des Gleichungssystems für die Randbedingungen der $N-1$ Splineabschnitte sowie der Ruck- und Abstandminimierung (2.5) und (2.6) mit anschließender numerischer Lösung anhand der Positionsstützpunkte $\left(x_{i}, y_{i}\right)$ und des Gewichtungsparameters $p$.

Die beispielhafte Sollbahnplanung für einen Doppelspurwechsels wird in Abbildung 2.2 gezeigt. Ausgangspunkt ist die durch die Manövervorgabe definierte Gasse eines Doppelspurwechsels. Die Kreise in der $x-y$-Ebene stellen die Stützpunkte $\mathbf{p}_{s}\left(t_{i}^{\star}\right)$ der synthetisch 
erzeugten Solltrajektorie innerhalb des gegebenen Korridors dar. Durch den konstanten Abstand benachbarter Punkte beeinhalten sie die Information der annähernd konstanten Fahrzeuggeschwindigkeit. Die durchgezogene Bahn in Abbildung 2.2 stellt die Sollbahn bei einem für dieses Manöver gewählten Parameter $p=1500$ in (2.5) und (2.6) dar. Sie entspricht der Sollbahn jenes Doppelspurwechsels, welcher später als Test für die entworfene Bahnfolgeregelung dient.

Während eines Doppelspurwechsels sorgt der Fahrer dafür, daß sich der Antriebsstrang des Fahrzeugs im Schleppbetrieb befindet. Das heißt, daß das Gaspedal in Ruhestellung, ein Gang eingelegt und die Kupplung geschlossen ist [13, 6]. Vor Beginn des Manövers stellt der Fahrer die gewünschte Anfangsgeschwindigkeit des Fahrzeugs ein. Während des Manövers wird der Geschwindigkeitsverlauf nur durch die Fahrwiderstände bestimmt und die Geschwindigkeit nimmt langsam ab. Da der genaue Geschwindigkeitsverlauf nicht im Voraus bekannt ist, wird in diesem Fall für den Entwurf der synthetischen Sollbahn und damit zur Minimierung des Rucks als Näherung eine konstante Sollgeschwindigkeit angenommen. Ist die Fahrzeuggeschwindigkeit während des Manövers kleiner als angenommen, fallen die Querbeschleunigung und der verbleibende Ruck geringer als geplant aus.

Bei konstanter Fahrzeuggeschwindigkeit entspricht eine Minimierung des Rucks einer Minimierung der Bahnkrümmungsänderung $\mathrm{d} \kappa / \mathrm{d} s$ bezüglich der Bogenlänge $s$ des Splines. Aus der geometrische Größe $\mathrm{d} \kappa / \mathrm{d} s$ resultiert, daß für Fahrdynamikmanöver die Anfangsgeschwindigkeit im Rahmen der Reifenhaftgrenze beliebig variiert werden kann, ohne die Ruckminimalität der Solltrajektorie zu beeinträchtigen. Ändert der Versuchsingenieur während der Versuchsreihe die Anfangsgeschwindigkeit des Manövers, entsprechen Ruck und Beschleunigung qualitativ weiterhin deren geplanten glatten Verlauf. Es ändert sich lediglich deren Amplitude. Der in [13] beschriebene doppelte Fahrspurwechsel, auch „Elchtest" genannt, ist ein typisches Beispiel für eine solche Versuchsreihe. Für dieses Manöver soll dieselbe Bahn bei steigender Anfangsgeschwindigkeit solange wiederholt befahren werden, bis das Fahrzeug der Bahn nicht mehr folgen kann.

Als Ergebnis der Planungsphase liegt ein Spline-Vektor

$$
\mathbf{p}_{s}\left(t^{\star}\right)=\left(s_{x}\left(t^{\star}\right), s_{y}\left(t^{\star}\right)\right) \text { mit } t_{1}^{\star} \leq t^{\star} \leq t_{N}^{\star}
$$

in der Ebene vor, der die Ortskurve der Sollbahn festlegt. Zu jedem Punkt $\mathbf{p}_{s}\left(t^{\star}\right)$ des Splines können aus dessen Komponenten $s_{x}\left(t^{\star}\right)$ und $s_{y}\left(t^{\star}\right)$ und deren ersten beiden zeitlichen Ableitungen (2.2) und (2.3) die dazugehörige Bewegungsrichtung $\theta\left(t^{\star}\right)$ mit (2.12) und Bahnkrümmung $\kappa\left(t^{\star}\right)$ mit (2.13) bestimmt werden. Diese Größen sind wie die Ortskurve der Sollbahn rein geometrischer Natur und nicht geschwindigkeitsabhängig.

In der Praxis wird für bestimmte Manövervorgaben jeweils ein eigener Spline (2.7) als Sollbahn festgelegt. Die Polynomkoeffizienten $a_{x i k}$ und $a_{y i k}$ jeder Sollbahn werden in einer 
Bibliothek abgelegt und können von dem Versuchsingenieur über die Bedienerschnittstelle des Mess- und Regelsystems ausgewählt werden. Trotz der vorgenommenen Umwandlung der zeitlichen Solltrajektorie des Fahrzeugschwerpunktes in eine örtliche Sollbahn soll die durch die Manövervorgabe vorgegebene Fahrzeuggeschwindigkeit bestmöglich eingehalten werden. Die Einstellung der Fahrzeuggeschwindigkeit ist durch alleinigen Einsatz der Lenkmaschine als Aktor nicht möglich und muß von dem Versuchsingenieur übernommen werden (Abbildung 2.1). Die Sollgeschwindigkeit ist dem Versuchsingenieur durch den Manöverwunsch bekannt und wird durch ihn während der Fahrt eingehalten.

Im folgenden Abschnitt wird beschrieben, wie der örtliche Ausgleichspline (2.7) entsprechend Abbildung 2.1 zusammen mit der aktuellen Fahrzeugposition und -geschwindigkeit für die Bestimmung der zeitabhängigen Solltrajektorie in Echtzeit verwendet wird. Mit der Solltrajektorie wird sowohl die Berechnung der Vorsteuerung als auch die Ermittlung der Regelabweichung und deren Ableitung für die Regelung durchgeführt.

\subsection{Echtzeitberechnung der Solltrajektorie}

Die Querregelung des Versuchsfahrzeugs betrifft den Abstand $d_{q}\left(t_{n}\right)$ des Fahrzeugschwerpunkts $\mathbf{p}\left(t_{n}\right)$ von der Sollposition $\mathbf{p}_{s}\left(t_{n}^{\star}\right)$ für jeden Abtastzeitpunkt $t_{n}$, wie dies in Abbildung 2.3 dargestellt ist. Dabei wird die Sollposition durch jenen Punkt $\mathbf{p}_{s}\left(t_{n}^{\star}\right)$ mit dem Splineparameter $t_{n}^{\star}$ auf der Sollbahn definiert, welcher dem Fahrzeugschwerpunkt $\mathbf{p}\left(t_{n}\right)$ geometrisch am nächsten ist. Da die gefahrene Fahrzeuggeschwindigkeit $v$ vom Versuchsingenieur abhängt, weicht sie in gewissen Grenzen von der Sollgeschwindigkeit $v_{s}$ ab. Als Folge stimmt die Zeit $t^{\star}$ der Sollbahnvorgabe nicht mit der Zeit $t$ des Versuchs überein. Damit die Sollposition $\mathbf{p}_{s}\left(t_{n}^{\star}\right)$ dennoch der dem Fahrzeugschwerpunkt nächste Punkt auf der Sollbahn ist, wird der Splineparameter $t_{n}^{\star}$ für jeden Abtastzeitpunkt $t_{n}$ in Abhängigkeit von der letzten gemessenen Fahrzeuggeschwindigkeit $v\left(t_{n-1}\right)$ mit der folgenden Formel angepaßt:

$$
t_{n}^{\star}=t_{n-1}^{\star}+\frac{v\left(t_{n-1}\right)}{v_{s}\left(t_{n-1}^{\star}\right)}\left(t_{n}-t_{n-1}\right)+p_{l} F\left(t_{n-1}\right) .
$$

Dabei korrigiert zunächst das Verhältnis $v\left(t_{n-1}\right) / v_{s}\left(t_{n-1}\right)$ von Ist- und Sollgeschwindigkeit das Inkrement $t_{n}^{\star}-t_{n-1}^{\star}$ des Splineparameters $t^{\star}$ zum Zeitpunkt $t_{n}$. Ist beispielsweise die Istgeschwindigkeit $v\left(t_{n-1}\right)$ größer als die Sollgeschwindigkeit $v_{s}\left(t_{n-1}^{\star}\right)$ aus (2.11), wächst der Splineparameter $t_{n}^{\star}$ schneller als nominell, damit der Fahrzeugschwerpunkt $\mathbf{p}\left(t_{n}\right)$ die Sollposition $\mathbf{p}_{s}\left(t_{n}^{\star}\right)$ nicht ,überholt".

Das mit (2.8) ermittelte Inkrement des Splineparameters $t_{n}^{\star}$ ist durch Messfehler in der Fahrzeuggeschwindigkeit $v\left(t_{n}\right)$ und kleine Abweichungen $d_{q}\left(t_{n}\right)$ des Fahrzeugs von der Sollbahn $\mathbf{p}_{s}\left(t_{n}^{\star}\right)$ fehlerbehaftet. Als Folge dieses Fehlers entsteht eine Längsabweichung

$$
d_{l}\left(t_{n}\right)=\frac{\mathbf{v}_{s}\left(t_{n}^{\star}\right)}{\left|\mathbf{v}_{s}\left(t_{n}^{\star}\right)\right|} \cdot\left[\mathbf{p}\left(t_{n}\right)-\mathbf{p}_{s}\left(t_{n}^{\star}\right)\right]
$$




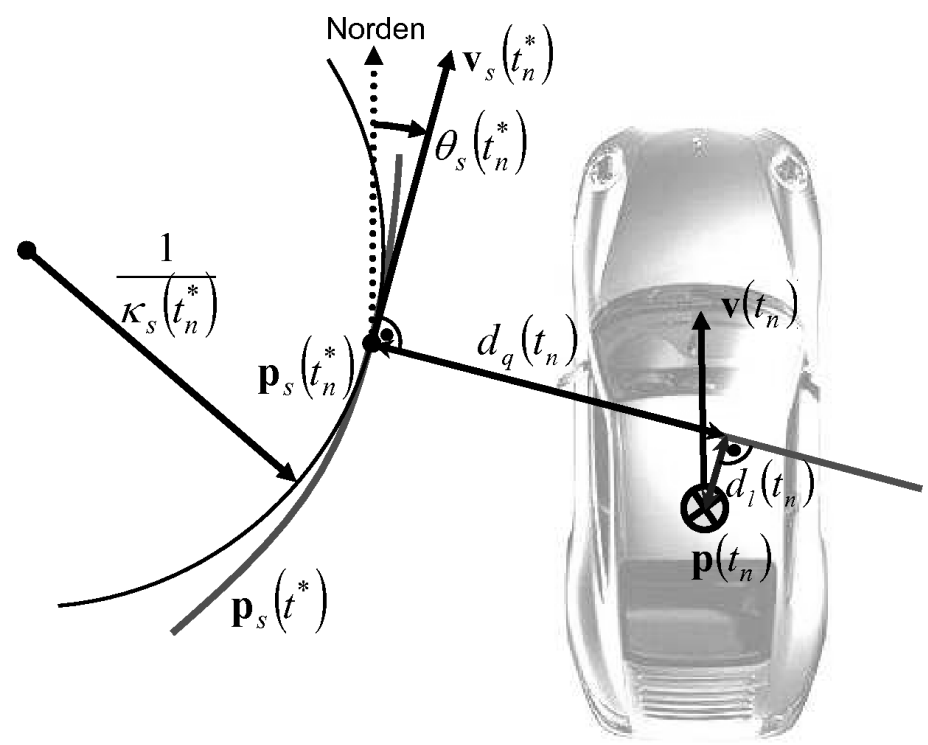

Abbildung 2.3: Geometrie von Soll- und Istposition $\mathbf{p}_{s}\left(t_{n}^{\star}\right)$ und $\mathbf{p}\left(t_{n}\right)$, Soll- und Istgeschwindigkeit $\mathbf{v}_{s}\left(t_{n}^{\star}\right)$ und $\mathbf{v}\left(t_{n}\right)$, Sollbewegungsrichtung $\theta_{s}\left(t_{n}^{\star}\right)$, Sollbahnkrümmung $\kappa_{s}\left(t_{n}^{\star}\right)$ und Abstand (Regelgröße) $d_{q}\left(t_{n}\right)$ im Abtastzeitpunkt $t_{n}$ sowie Darstellung der Sollbahn $\mathbf{p}_{s}\left(t^{\star}\right)$ mit dem Splineparameter $t^{\star}$ bzw. $t_{n}^{\star}$.

zwischen der Sollposition $\mathbf{p}_{s}\left(t_{n}^{\star}\right)$ und dem Fahrzeugschwerpunkt $\mathbf{p}\left(t_{n}\right)$, was im letzten Summanden von (2.8) berücksichtigt wird. Der Quotient

$$
F\left(t_{n-1}\right)=-d_{l}\left(t_{n-1}\right) / v_{s}\left(t_{n-1}^{\star}\right)
$$

ist der Fehler von $t_{n}^{\star}$, der zu der Längsabweichung $d_{l}\left(t_{n}\right)$ führt. Durch den heuristisch gewählten Gewichtungsparameter $0<p_{l}<1$ in (2.8) wird der Splineparameter $t_{n}^{\star} \mathrm{zu}$ jedem Abtastzeitpunkt so korrigiert, daß sich die Längsabweichung $d_{l}\left(t_{n}\right)$ in tolerierbaren Grenzen bewegt. Ist die Messung ohne Rauschen, könnte $p_{l}=1$ gesetzt werden. Um eine ruhigere Korrektur zu erzielen, wird durch $p_{l}<1$ eine kleine bleibende Abweichung in Kauf genommen.

Durch den auf diese Weise bestimmten Splineparameter $t_{n}^{\star}$ ist keine iterative Suche des nächstgelegenen Punkts auf der Sollbahn nötig und die benötigte Rechenzeit bleibt planbar. Mit Hilfe des zum Zeitpunkt $t_{n}$ gehörigen Splineparameters $t_{n}^{\star}$ lassen sich aus dem Spline (2.1) und dessen Ableitung (2.2) die zu jedem Abstastzeitpunkt $t_{n}$ gehörende Sollposition $\mathbf{p}_{s}\left(t_{n}^{\star}\right)$ und Sollgeschwindigkeit

$$
\mathbf{v}_{s}\left(t_{n}^{\star}\right)=\left(s_{x}^{\prime}\left(t_{n}^{\star}\right), s_{y}^{\prime}\left(t_{n}^{\star}\right)\right)
$$

berechnen. Aus den Komponenten $\left(s_{x}^{\prime}\left(t_{n}^{\star}\right), s_{y}^{\prime}\left(t_{n}^{\star}\right)\right)$ des Sollgeschwindigkeitsvektors (2.11) wird die Sollbewegungsrichtung

$$
\theta_{s}\left(t_{n}^{\star}\right)=\arctan 2\left(s_{y}^{\prime}\left(t_{n}^{\star}\right), s_{x}^{\prime}\left(t_{n}^{\star}\right)\right)
$$


Trajektorienplanung für die Steuerung und Regelung

berechnet ${ }^{3}$.

Die von der Vorsteuerung benötigte Sollbahnkrümmung $\kappa_{s}\left(t_{n}^{\star}\right)$ ist als Kehrwert des Bahnradius wie die Sollbewegungsrichtung $\theta_{s}\left(t_{n}^{\star}\right)$ eine geometrische Eigenschaft der Sollbahn an dem Punkt $\mathbf{p}_{s}\left(t_{n}^{\star}\right)$. Aus den Ableitungen (2.2) und (2.3) der $x$ - und $y$-Komponenten des Ausgleichssplines für den zum Zeitpunkt $t_{n}$ gehörigen Splineparameter $t_{n}^{\star}$ wird die Sollbahnkrümmung

$$
\kappa_{s}\left(t_{n}^{\star}\right)=\frac{\theta_{s}^{\prime}\left(t_{n}^{\star}\right)}{\left|\mathbf{v}\left(t_{n}^{\star}\right)\right|}=\frac{s_{x}^{\prime}\left(t_{n}^{\star}\right) s_{y}^{\prime \prime}\left(t_{n}^{\star}\right)-s_{x}^{\prime \prime}\left(t_{n}^{\star}\right) s_{y}^{\prime}\left(t_{n}^{\star}\right)}{\left(s_{x}^{\prime 2}\left(t_{n}^{\star}\right)+s_{y}^{\prime 2}\left(t_{n}^{\star}\right)\right)^{3 / 2}}
$$

berechnet.

Mit der gemessenen Position $\mathbf{p}\left(t_{n}\right)$, der Sollposition $\mathbf{p}_{s}\left(t_{n}^{\star}\right)$ und der Sollbewegungsrichtung $\theta_{s}\left(t_{n}^{\star}\right)$ wird die Regelabweichung

$$
d_{q}\left(t_{n}\right)=\left[-\sin \theta_{s}\left(t_{n}^{\star}\right), \cos \theta_{s}\left(t_{n}^{\star}\right)\right] \cdot\left[\mathbf{p}\left(t_{n}\right)-\mathbf{p}_{s}\left(t_{n}^{\star}\right)\right]
$$

als Projektion des Abstandsvektors $\left[\mathbf{p}\left(t_{n}\right)-\mathbf{p}_{s}\left(t_{n}^{\star}\right)\right]$ auf den zur Sollbewegungsrichtung $\theta_{s}\left(t_{n}^{\star}\right)$ orthogonalen Einheitsvektor $\left[-\sin \theta_{s}\left(t_{n}^{\star}\right), \cos \theta_{s}\left(t_{n}^{\star}\right)\right]$ bestimmt (Abbildung 2.3).

Die Regelung benötigt neben der Regelabweichung $d_{q}\left(t_{n}\right)$ auch deren zeitliche Ableitung, d.h. die Annäherungsgeschwindigkeit $\dot{d}_{q}\left(t_{n}\right)$. Um eine numerische Differentiation zur Berechnung von $\dot{d}_{q}\left(t_{n}\right)$ zu vermeiden, wird aus der Differenz von Sollbewegungsrichtung $\theta_{s}\left(t_{n}^{\star}\right)$ aus (2.12) und gemessener Bewegungsrichtung $\theta\left(t_{n}\right)$ des Fahrzeugschwerpunktes zusammen mit der gemessenen Fahrzeuggeschwindigkeit $v\left(t_{n}\right)$ die Geschwindigkeit

$$
\dot{d}_{q}\left(t_{n}\right)=-v\left(t_{n}\right) \sin \left(\theta_{s}\left(t_{n}^{\star}\right)-\theta\left(t_{n}\right)\right) .
$$

berechnet. Da die Erfassung der Fahrzeuggeschwindigkeit und -bewegungsrichtung durch Integration der gemessenen Beschleunigung und Drehrate des Fahrzeugs erfolgt (siehe Kapitel 3), ist die durch (2.15) berechnete Annäherungsgeschwindigkeit $\dot{d}_{q}\left(t_{n}\right)$ in der Praxis sehr rauscharm.

\subsection{Neuplanung der Trajektorie bei großen Regelab- weichungen}

Im realen Fahrversuch kann es vorkommen, daß der Querabstand $d_{q}$ zu groß wird (hier: größer als ein Meter). Ein Grund dafür kann die Initialisierung der Bahnfolgeregelung zu einem Zeitpunkt sein, zu dem sich das Fahrzeug nicht auf der Sollbahn befindet. Eine

\footnotetext{
${ }^{3}$ Die Funktion $\arctan 2(y, x)$ unterscheidet sich von der Funktion $\arctan (y / x)$ dadurch, daß die Lösung unter Beachtung der Vorzeichen von $y$ und $x$ in dem Intervall $[-\pi, \pi]$ definiert ist, und nicht auf das Intervall $[-\pi / 2, \pi / 2]$ beschränkt bleibt.
} 


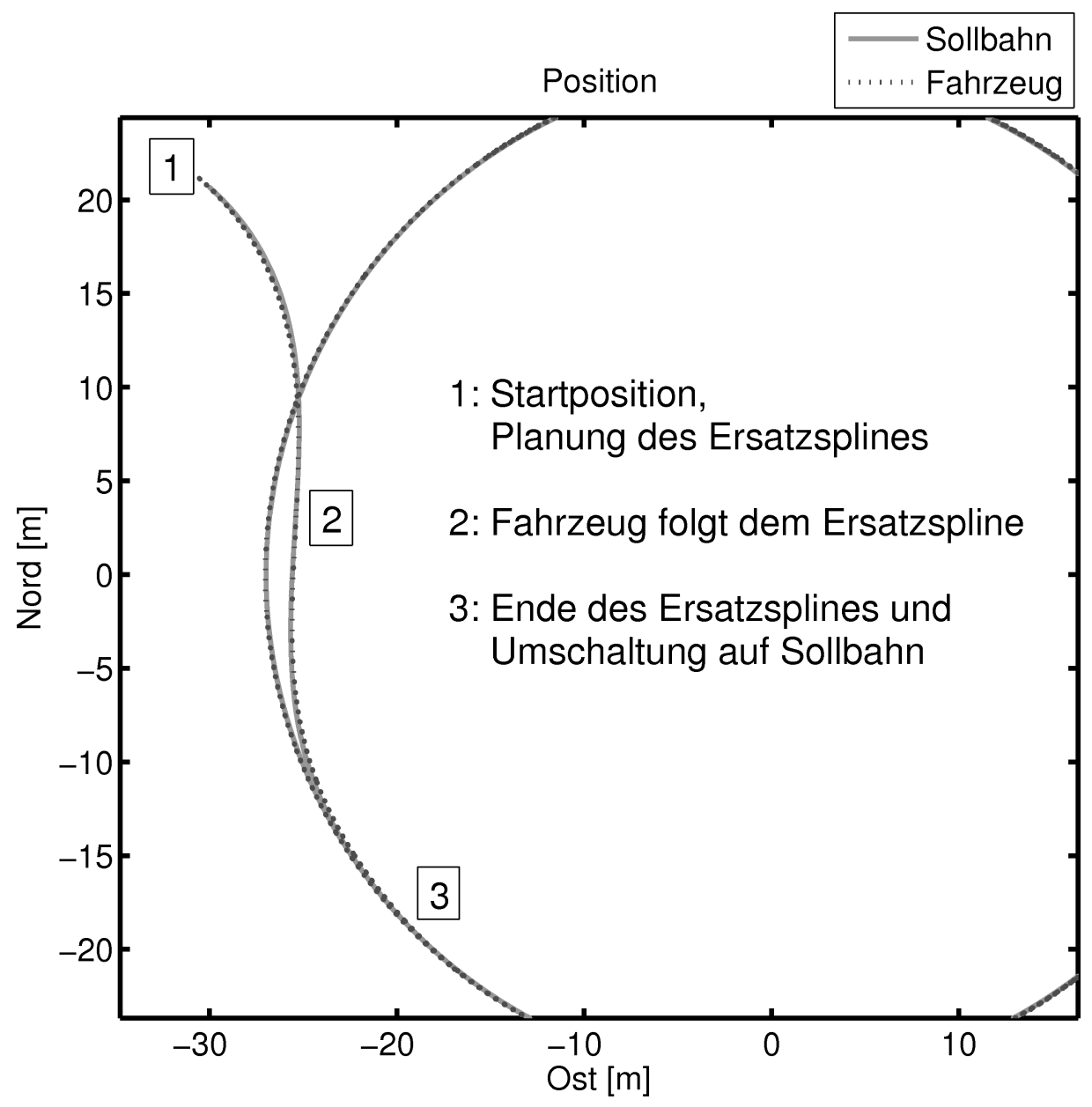

Abbildung 2.4: Planung eines Ersatzsplines zur Sollbahn bei Initialisierung der Bahnfolgeregelung

Überschreitung der maximal möglichen Querbeschleunigung während der Fahrt wegen zu hoher Geschwindigkeit kann ebenfalls ein Grund für einen zu großen Querabstand sein. Um eine jederzeit einwandfreie Funktion der Bahnfolgeregelung zu ermöglichen, muß für diesen Fall eine geeignete Ersatz-Sollbahn in Echtzeit erzeugt werden, die temporär als Sollbahndefinition für die Berechnung der Solltrajektorie eingesetzt wird. In den beiden folgenden Abschnitten wird die Planung eines Ersatzsplines bei Überschreitung eines Querabstandes von einem Meter am Beispiel einer kreisförmigen Sollbahn dargestellt.

\subsubsection{Splineplanung zu Beginn der Fahrt}

In der Praxisanwendung befindet sich das Fahrzeug bei Initialisierung der Regelung in der Tat meist nicht genau auf der Sollbahn. Der Ersatzspline führt das Fahrzeug unter Berücksichtigung der aktuellen Fahrzeugposition und -orientierung auf die Sollbahn. Er 
besitzt dieselbe parametrische Darstellung wie ein Segment der Sollbahn. Dadurch ist es möglich, ohne Änderung der Echtzeitberechnung für die Solltrajektorie den Datensatz der Sollbahn temporär zu ersetzen.

Ein Beispiel für die Initialisierung der Bahnfolgeregelung ist in Abbildung 2.4 dargestellt. An der Markierung (1) befindet sich das Fahrzeug zunächst im Stillstand und die Bahnfolgeregelung wird aktiviert. Da die Querabweichung von der Sollbahn größer als ein Meter ist, wird ein Ersatzspline geplant, der die Fahrzeugposition mit der Sollbahn verbindet. Das Fahrzeug fährt an und verfolgt den Ersatzspline (2). Am Endpunkt des Ersatzsplines (3) wird für die weitere Trajektorienplanung auf die ursprünglich geplante Sollbahn umgeschaltet.

\subsubsection{Splineplanung beim Überschreiten der Reifenhaftungs- grenze}

Wird die Abweichung von der Sollbahn aufgrund einer Überschreitung der physikalischen Grenzen größer als der definierte Grenzwert von zum Beispiel einem Meter, wird die Sollbahn temporär verworfen, um durch eine neue Sollvorgabe ersetzt zu werden. Diese besteht aus einem Spline, dessen Anfang mit der aktuellen Position, Bewegungsrichtung und Bahnkrümmung des Fahrzeugschwerpunktes übereinstimmt. Dadurch wird weiter das Maximum an Reifenführungskraft gefordert und das Fahrzeug schnellstmöglich zur eigentlichen Sollbahn zurückgeführt. Das Ende des Ersatzsplines liegt auf der Sollbahn mit deren Krümmung und Sollbewegungsrichtung. Der Ersatzssplines soll das Fahrzeug innerhalb einer Sekunde auf die Sollbahn zurückführen. Daher ist dessen Länge geschwindigkeitsabhängig.

Ein Beispiel einer solchen Neuplanung der Sollbahn ist in Abbildung 2.5 dargestellt. Das Fahrzeug verfolgt die kreisförmige Sollbahn gegen den Uhrzeigersinn. Die Geschwindigkeit wird langsam erhöht (1). Bei Überschreitung der maximal möglichen Geschwindigkeit kann das Fahrzeug nicht mehr auf der Sollbahn gehalten werden. Der Kreisradius vergößert sich (2). Bei Überschreitung des maximalen Abstandes zwischen Fahrzeugschwerpunkt und Sollbahn wird eine neue Sollbahn geplant, die vom Fahrzeugschwerpunkt zur eigentlichen Sollbahn zurückführt (3). Solange die Geschwindigkeit nicht reduziert wird, wiederholt sich die Neuplanung bei jeder weiteren Überschreitung des maximalen Querabstands (4). Die Querbeschleunigung bleibt im Maximum, bis die Sollbahn durch Reduzierung der Geschwindigkeit wieder erreicht werden kann (5). Ist das Ende des Ersatzsplines erreicht (6), wird der dazugehörige Parametersatz gelöscht, so daß wieder die eigentlich geplante Sollbahn für die Erzeugung der Solltrajektorie verwendet wird. 


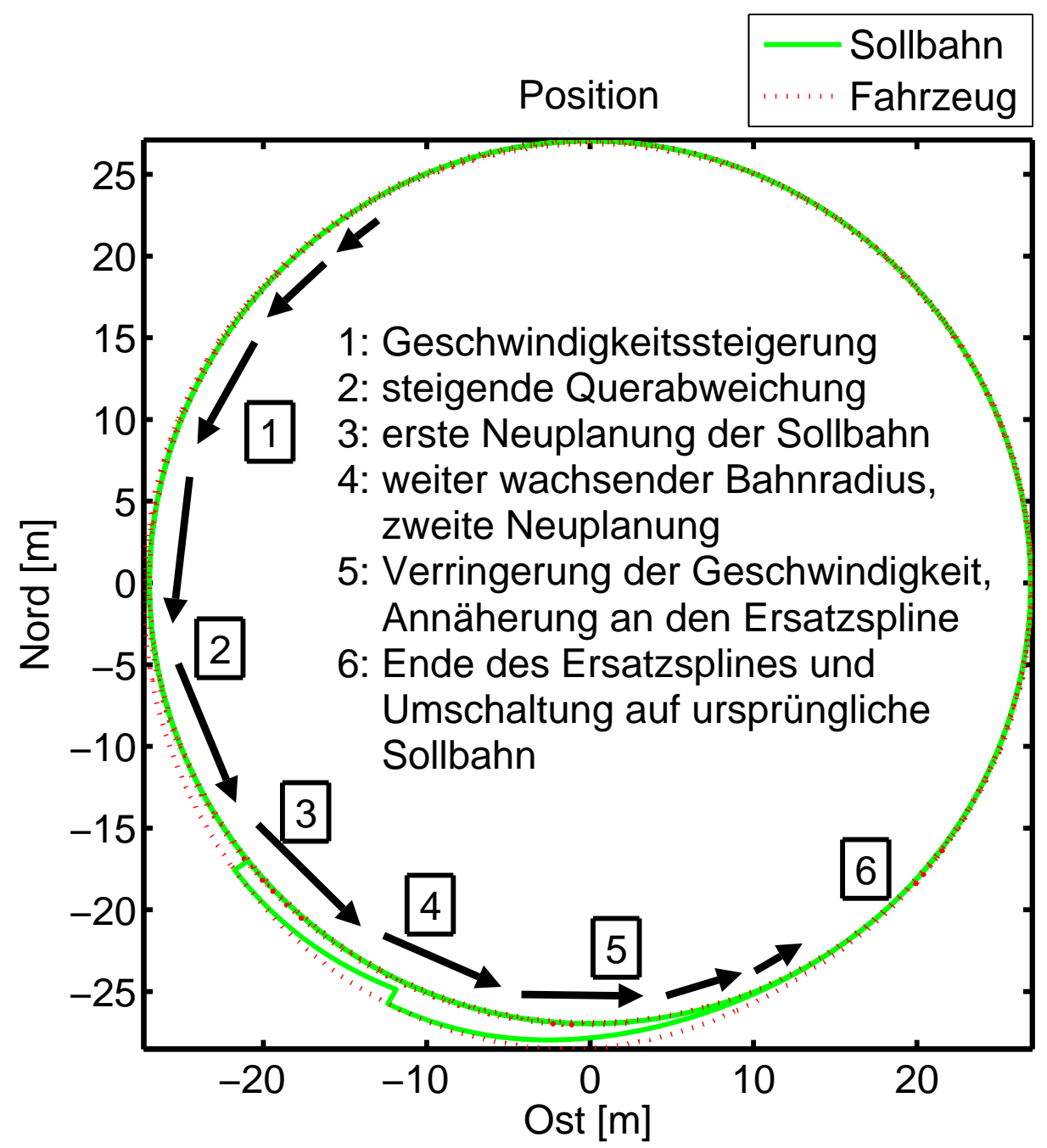

Abbildung 2.5: Neuplanung der Sollbahn bei zu großer Querabweichung durch Überschreitung der Reifenhaftungsgrenze am Beispiel der Kreisfahrt 
Trajektorienplanung für die Steuerung und Regelung

\subsection{Zusammenfassung}

In diesem Kapitel wird erläutert, wie entsprechend Abbildung 2.1 aus einem durchzuführenden Manöver zunächst eine einheitliche Manövervorgabe, dann eine glatte und ruckarme örtliche Sollbahn und schließlich eine zu der aktuellen Fahrzeuggeschwindigkeit und -position des Fahrzeugs passende örtliche und zeitliche Solltrajektorie für die Vorsteuerung und Regelung gewonnen wird. Die Umwandlung des gewünschten Manövers in eine örtliche Sollbahn geschieht im Vorfeld der Versuche. In dieser Planungsphase wird das gewünschte Manöver zunächst wie genannt in die einheitliche Form einer Manövervorgabe gebracht und anschließend unter Minimierung des Rucks durch einen Splinevektor angenähert. Die Ordnung der Splines ist so gewählt, daß die vorgegebene Bahn durch das Fahrzeug realisierbar ist. Als wichtiges Resultat der Planungsphase steht die Sollbahn in Form ihrer Polynomkoeffizienten $a_{x i k}$ und $a_{y i k}$ in (2.1) zur Verfügung. Diese Darstellung der örtlichen Sollbahn ermöglicht eine effiziente Weiterverarbeitung während der Fahrt zur Gewinnung der Solltrajektorie.

Im Echtzeitteil wird der Sollzeitparameter $t_{n}^{\star}$ zu jedem Abtastzeitpunkt $t_{n}$ anhand der gemessenen Fahrzeugposition- und Geschwindigkeit so nachgeführt, daß die Sollposition $\mathbf{p}_{s}\left(t_{n}^{\star}\right)$ einen annähernd minimalen Abstand zu der gemessenen Position $\mathbf{p}\left(t_{n}\right)$ des Fahrzeugschwerpunktes hat. Dieser Abstand ist gleichzeitig die Regelabweichung $d_{q}\left(t_{n}\right)$ und wird dem Regler übergeben. Mit $t_{n}^{\star}$ wird die zu jedem Abtastzeitpunkt $t_{n}$ und der Sollposition $\mathbf{p}_{s}\left(t_{n}^{\star}\right)$ gehörige Sollgeschwindigkeit $v_{s}\left(t_{n}^{\star}\right)$, Sollbewegungsrichtung $\theta_{s}\left(t_{n}^{\star}\right)$ und Sollbahnkrümmung $\kappa_{s}\left(t_{n}^{\star}\right)$ aus dem Splinevektor ermittelt. Zusammen mit der gemessenen Bewegungsrichtung und Geschwindigkeit des Fahrzeugschwerpunktes wird eine rauscharme Bestimmung der Annäherungsgeschwindigkeit $\dot{d}_{q}\left(t_{n}\right)$ erreicht, welche zusammen mit der Abweichung $d_{q}\left(t_{n}\right)$ für die Regelung benötigt wird. Die Sollbahnkrümmung $\kappa_{s}\left(t_{n}^{\star}\right)$ wird von der Vorsteuerung benötigt.

Falls die Fahrzeugposition zu weit von der Sollbahn abweicht, sei es bei Aktivierung der Bahnfolgeregelung oder durch Überschreitung der Reifenhaftgrenze bei Kurvenfahrt, wird ein Ersatzspline geplant, der das Fahrzeug an die Sollbahn zurückführt. So ist für die Bahnfolgeregelung sichergestellt, daß sich das Fahrzeug immer in der Nähe einer geeigneten glatten Sollbahn befindet.

Für den Praxisversuch des Gesamtsystems der Querregelung mit der in diesem Kapitel beschriebenen Trajektorienplanung wird eine echtzeitfähige Implementierung der Trajektorienplanung auf fahrzeugtauglicher Hardware benötigt. Diese wird in Abschnitt 5.1.2 beschrieben.

Bei der beschriebenen Bahn- und Trajektorienplanung wird die Kenntnis der tatsächlichen Bewegungsrichtung $\theta\left(t_{n}\right)$, Geschwindigkeit $v\left(t_{n}\right)$ und Position $\mathbf{p}\left(t_{n}\right)$ des Fahrzeugschwerpunktes vorausgesetzt. Für die genaue Messung dieser Größen während 
der Fahrt in Echtzeit wird das im folgenden Kapitel beschriebene echtzeitfähige integrierte Navigationssystem verwendet. 


\section{Kapitel 3}

\section{Messung der Fahrzeugbewegung}

Für die objektive Auswertung von Fahrdynamikversuchen muß die Bewegung der Karosserie im Raum messtechnisch erfaßt werden. Dafür steht ein integriertes, d.h. hier ein satellitengestütztes inertiales Navigationssystem zur Verfügung [36, 7, 20]. Ein solches Navigationssystem zeichnet sich durch sehr gute Verfügbarkeit, hohe Genauigkeit und eine große Datenrate aus. Zu der Fahrzeugbewegung zählt in diesem Fall sowohl die Trajektorie des Fahrzeugschwerpunktes mit Position, Geschwindigkeit und Beschleunigung als auch die Orientierung und Winkelgeschwindigkeit der Karosserie. Die genannten Größen sind jeweils Vektoren im dreidimensionalen Raum. Sie bilden die Basis für die objektive Auswertung der Fahrversuche.

Die durch das Navigationssystem bereitgestellten Messgrößen stehen auch für die Bahnfolgeregelung des Fahrzeugs zur Verfügung. Daher kann das Navigationssystem neben der Versuchsauswertung auch der Regelung dienen (Abbildung 1.2) ohne daß weiterer Aufwand bei der Ausrüstung des Fahrzeugs mit Messtechnik entsteht. Die Regelung stellt im Unterschied zu der Versuchsauswertung Echtzeitanforderungen an die Messtechnik. Für eine reine Auswertung der Fahrversuche reicht hingegen die Aufzeichnung der Sensorrohwerte und eine anschließende Navigationsrechnung aus.

In diesem Kapitel werden die Funktionsweise des verwendeten echtzeitfähigen satellitengestützten inertialen Navigationssystems und die Gewinnung der für die Regelung benötigten Größen erläutert. Abschnitt 3.1 befasst sich mit der Definition der verwendeten Koordinatensysteme, der Berechnung der Fahrzeugbewegung aus den Messungen der Inertialsensoren und der Anfangsausrichtung, d.h. der Initialisierung der Navigationsrechnung. Um die Langzeitstabilität des Navigationssystems zu gewährleisten, werden die Ergebnisse der Navigationsrechnung und die Signale des Satellitennavigationssystems über ein Kalman-Filter zusammengeführt (Abschnitt 3.2). In Abschnitt 3.3 wird der Ausgang des integrierten Navigationssystems verwendet, um die für die Steuerung und Regelung benötigten fahrdynamikrelevanten Größen zu berechnen. 

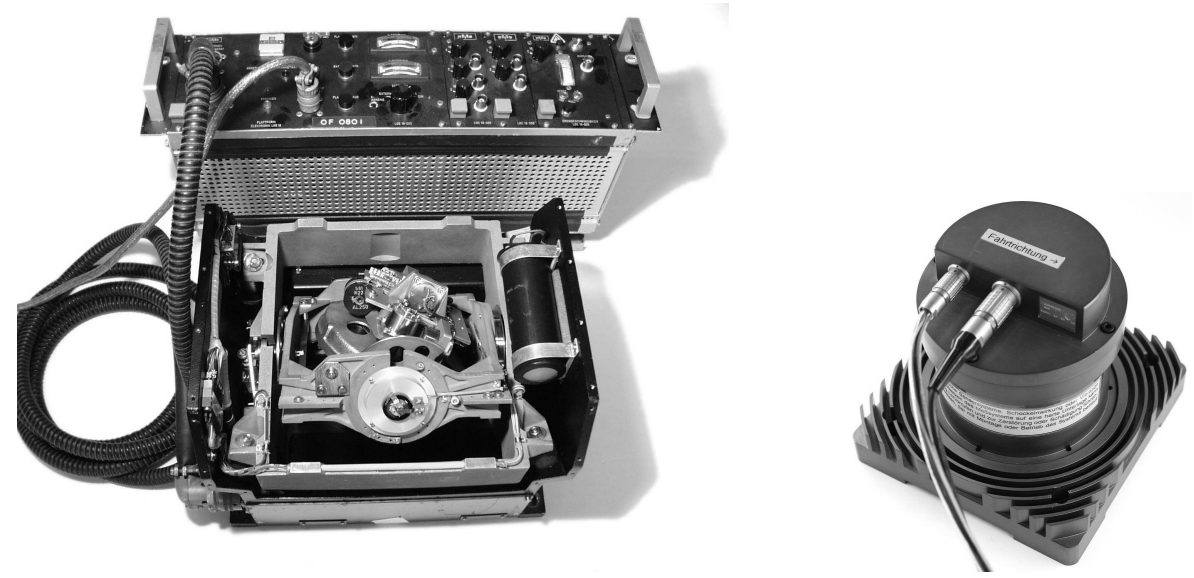

Abbildung 3.1: Links die Darstellung einer Trägheitsplattform mit kardanisch aufgehängten und kreiselstabilisierten Beschleunigungssensoren der Firma RMS Testsystems. Rechts im nicht maßstäblichen Vergleich das verwendete dreiachsiges Inertialsensorpaket LN-200 der Firma Northrop-Grumman mit faseroptischen Kreiseln und ServoBeschleunigungsaufnehmern für die Fahrdynamikmessungen.

\subsection{Inertiales Navigationssystem}

Das inertiale Navigationssystem (Abk.: INS ${ }^{1}$ ) basiert im Prinzip auf der für ebene Probleme verwendeten Koppelnavigation ${ }^{2}$. Bei dieser Navigationsmethode wird aus der in bekannten Zeitabständen gemessenen Geschwindigkeit die Position berechnet [36]. Das inertiale Navigationssystem überträgt dieses Prinzip in den dreidimensionalen Raum und auf die Messung der Beschleunigung. Für die direkte Berechnung des Geschwindigkeitsvektors und der Position in einem definierten Koordinatensystem müssen die Sensorachsen ihre Orientierung bezüglich des Koordinatensystems beibehalten. Für Fahrdynamikmessungen werden dafür die Beschleunigungssensoren auf einer kardanisch gelagerten, kreiselstabilisierten Plattform angebracht. So behalten die Sensoren während der Fahrt ihre Winkellage im Raum unabhängig von der Bewegung des Fahrzeugs bei. Abbildung 3.1 links zeigt eine Ansicht dieser Trägheitsplattform. Die dazu nötige empfindliche Mechanik ist in der Fahrzeugerprobung fehleranfällig und wird daher heute bevorzugt durch ein sogenanntes „Strapdown“-Navigationssystem³ ersetzt. In der Strapdown-Systematik sind die Beschleunigungssensoren fest mit dem Fahrzeug verbunden und registrieren somit die Beschleunigungen zwangsläufig in dem Körperkoordinatensystem des Fahrzeugs. Ein solches Sensorpaket ist in Abbildung 3.1 rechts gezeigt. Die bei einem kardanische aufgehängten Kreiselsystem mechanisch realisierte Ausrichtung zum Bezugssystem wird hier durch eine rechnerische Koordinatentransformation ersetzt. Die Orientierung des

\footnotetext{
${ }^{1}$ INS: Inertial Navigation System

${ }^{2}$ eng.: dead-reckoning; ursprünglich „ded-reckoning“, Abkürzung für „deduced reckoning“.

3 „strap down": engl. für „festschnallen“
} 


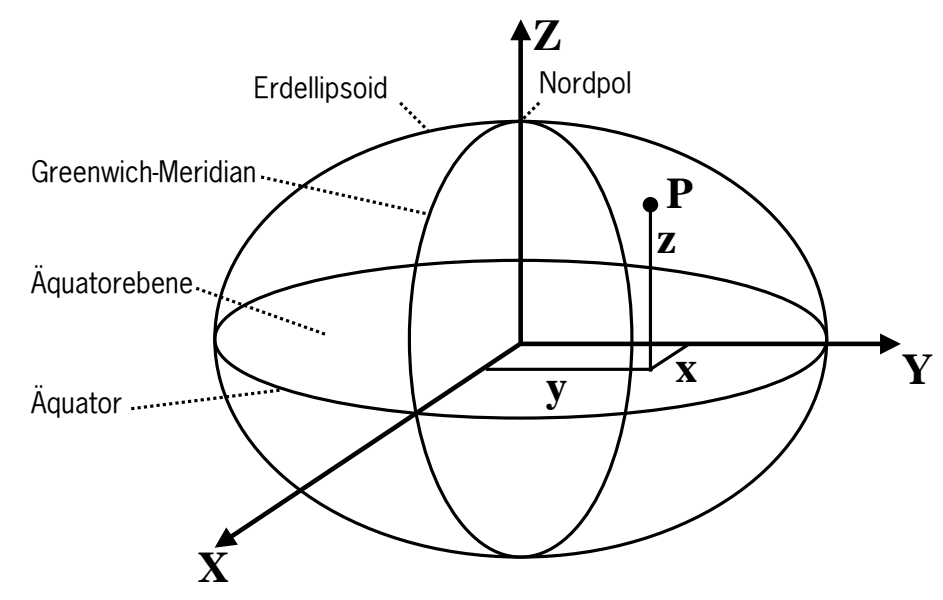

Abbildung 3.2: Position $\mathbf{P}(x, y, z)$ im Erd- bzw. im „earth-centered, earth-fixed“ (ECEF)-Koordinatensystem mit Ursprung im Erdmittelpunkt und den rechtwinkligen Achsen in der Äquatorebene und in Richtung Nordpol

Fahrzeugkoordinatensystems im Bezugssystem wird dann zur Ermittlung der zugehörigen Transformationsmatrix über ebenfalls mit dem Fahrzeug fest verbundene faseroptische Drehratensensoren erfaßt.

\subsubsection{Koordinatensysteme}

Die Strapdown-Navigationsrechnung verwendet demzufolge verschiedene Koordinatensysteme, die nachfolgend definiert werden. Die Transformation eines Vektors $\mathbf{v}^{\Phi_{1}}$ im Koordinatensystem $\Phi_{1}$ in das Koordinatensystem $\Phi_{2}$ kann mithilfe einer orthonormalen Richtungscosinusmatrix $\mathbf{C}$ mit $\mathbf{C}^{T} \mathbf{C}=\mathbf{C C}^{T}=\mathbf{I}$ erfolgen [7]:

$$
\mathbf{v}^{\Phi_{2}}=\mathbf{C}_{\Phi_{1}}^{\Phi_{2}} \mathbf{v}^{\Phi_{1}}
$$

Das inertiale Bezugssystem i ist unbeschleunigt, kann sich aber in gleichförmiger Bewegung befinden. Der Ursprung und die Orientierung sind beliebig wählbar, aber am Fixsternhimmel auszurichten. Die Beschleunigungssensoren und Drehratensensoren messen bezüglich dieses Systems; daher der Name „Inertialsensor“. Um die Auswirkungen der Drehung der Erde um die eigene Achse und um die Sonne zu berücksichtigen, wird im Folgenden angenommen, daß unser Sonnensystem unbeschleunigt und damit ein inertiales Bezugssystem ist.

Das Erdkoordinatensystem e (Abbildung 3.2) hat seinen Ursprung im Mittelpunkt der Erde. Die $\mathrm{x}$-Achse liegt in der terrestrischen Äquatorebene in Richtung des Nullmeridians. Die z-Achse ist parallel zur Achse der Erddrehung. Die y-Achse komplet- 


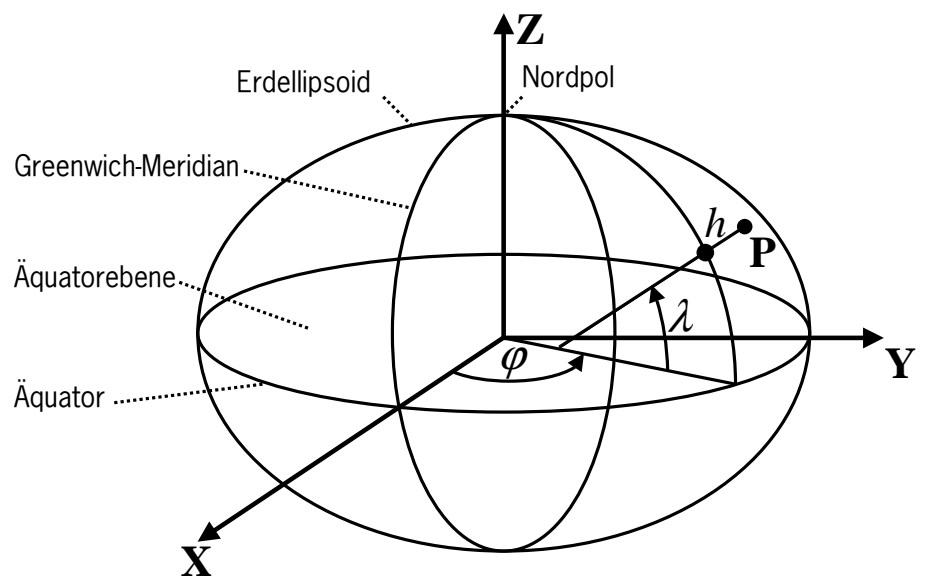

Abbildung 3.3: Position $\mathbf{P}(\lambda, \varphi, h)$ im geodätischen Koordinatensysten $g$ mit Breitengrad $\lambda$, Längengrad $\varphi$ und Höhe $h$ über dem Ellipsoid

tiert das rechtwinklige Koordinatensystem und schneidet den Äquator und den neunzigsten Längengrad [39]. Die Achsen ändern ihre Lage bezüglich des Inertialsystems $i$ hauptsächlich durch die Erddrehung mit einer Drehrate von $\omega_{i e} \approx 15.04109^{\circ} / \mathrm{h}[20]$.

Das geodätische Koordinatensystem g beschreibt die Position eines Raumpunktes über die geografische Breite $\lambda$ mit $\lambda \in[-\pi / 2, \pi / 2]$, geografische Länge $\varphi, \varphi \in[0,2 \pi]$ und ellipsoidische Höhe $h$ (Abbildung 3.3) [39]. Die geodätischen Koordinaten $(\lambda, \varphi, h)$ lassen sich in Erdkoordinaten $e$ überführen und umgekehrt [36].

Das Navigationskoordinatensystem $\mathbf{n}$ beschreibt eine Ebene, die an der Fahrzeugposition parallel zu der Oberfläche des Erdellipsoids ist. Der Ursprung der rechtwinkligen Achsen ist der Fahrzeugschwerpunkt. Der bewegliche Ursprung $\mathbf{P}$ wird durch seine geodätischen Koordinaten Breitengrad $\lambda$, Längengrad $\varphi$ und Höhe $h$ über dem Ellipsoid repräsentiert. Die Achsen zeigen in Richtung Norden, Osten und ins Erdinnere „northeast-down" (NED) (Abbildung 3.4).

Das lokale ebene Koordinatensystem $\mathbf{t}$ wird in der Fahrdynamikanwendung verwendet. Der Unterschied zu dem Navigationskoordinatensystem liegt darin, daß der Ursprung $\mathbf{P}_{\mathbf{0}}$ nicht im Fahrzeugschwerpunkt, sondern erdfest im Mittelpunkt des Prüfgeländes liegt (Abbildung 3.5). Die rechtwinkligen Achsen zeigen in Richtung Norden, Osten und ins Erdinnere (NED). Die Koordinaten des Fahrzeugschwerpunktes $P$ werden in kartesischen Koordinaten mit der Einheit „Meter" angegeben. Diese Koordinaten sind gleichzeitig auch die Koordinaten für die Querregelung. Die Sollbahn der Steuerung und 


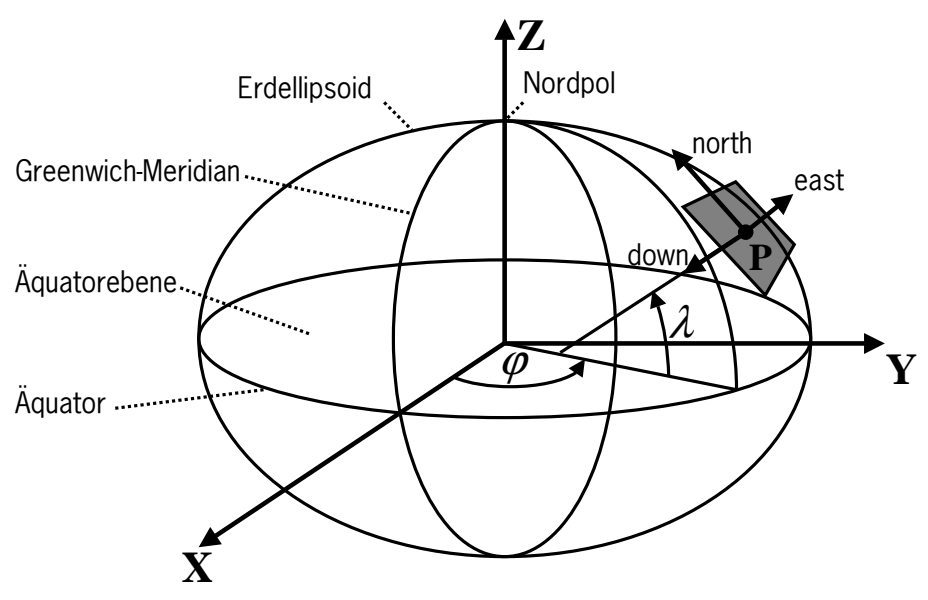

Abbildung 3.4: Kartesisches Navigationskoordinatensystem $n$ mit Nullpunkt $\mathbf{P}$ und den Achsrichtungen „north-east-down“ (NED)

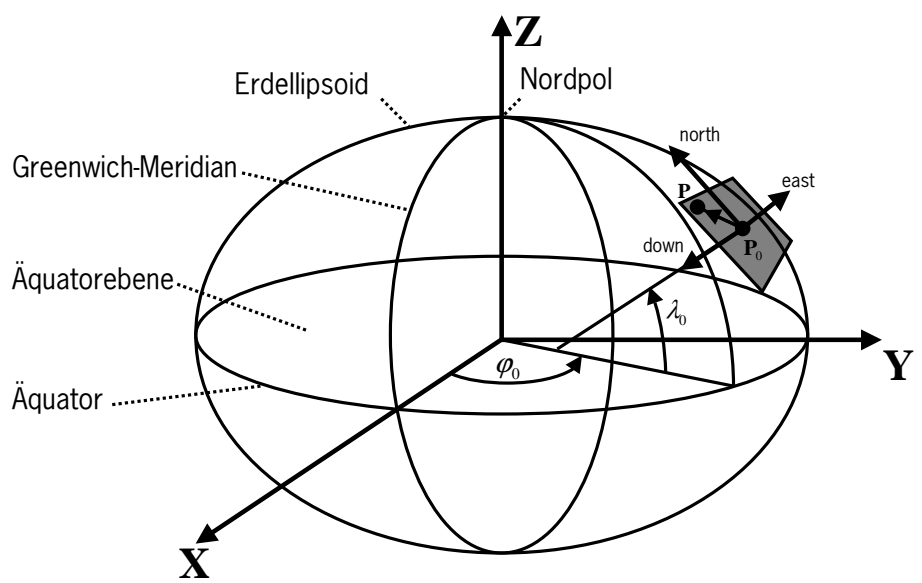

Abbildung 3.5: Lokales ebenes Koordinatensystem $t$ mit dem festen Ursprung $\mathbf{P}_{\mathbf{0}}$ und der Position $\mathbf{P}$ in kartesischen Koordinaten. 


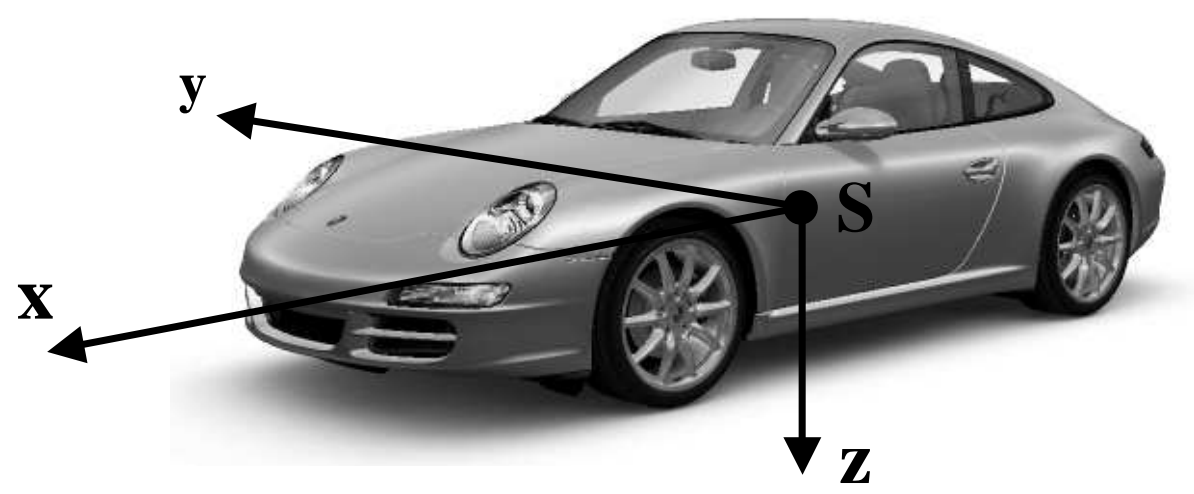

Abbildung 3.6: Körperkoordinatensystem $b$ mit den kartesischen Achsen $(\mathbf{x}, \mathbf{y}, \mathbf{z})$ und dem Ursprung im Fahrzeugschwerpunkt $\mathbf{S}$ oder im Messzentrum des Inertialsensorblocks.

Regelung liegt durch Vernachlässigung der Höheninformation in der Nord-Ost-Ebene des lokalen ebenen Koordinatensystems $t$.

Das Körperkoordinatensystem b ist fest mit dem Fahrzeug verbunden. Die kartesischen Achsen (x, y, z) zeigen nach vorne, rechts und nach unten (Abbildung 3.6). Das inertiale Sensorpaket und somit auch die Sensorachsen sind an diesem Koordinatensystem ausgerichtet. Die Inertialsensoren liefern daher die Messung in Körperkoordinaten. Für die rechnerischen Ausrichtung der Sensorachsen an dem Navigationskoordinatensystem $n$ und für die anschließende numerische Integration der gemessenen Beschleunigung zu Geschwindigkeit und Position wird die sogenannte Strapdown-Navigationsrechnung verwendet.

\subsubsection{Strapdown-Navigationsrechnung}

Das Blockschaltbild aus [29] in Abbildung 3.7 zeigt eine Übersicht der wesentlichen Rechenschritte der Strapdown-Rechnung [35, 7]. Die von dem Inertialsensorpaket (Abk.: IMU ${ }^{4}$ ) gemessene Drehrate $\omega_{i b}^{b}$ des Fahrzeugs bezüglich des Inertialsystems $i$ in Körperkoordinaten $b$ wird um die Drehbewegung $\omega_{i n}^{b}$ des Navigationskoordinatensystems $n$ bezüglich des Inertialsystems $i$ korrigiert, um die Orientierungsänderung

$$
\omega_{n b}^{b}=\omega_{i b}^{b}-\omega_{i n}^{b}
$$

des Fahrzeugs bezüglich des Navigationskoordinatensystems $n$ zu erhalten. Eine anschließende zeitliche Integration von $\omega_{n b}^{b}$ liefert als Ergebnis die aktuelle Orientierungsmatrix $\mathbf{C}_{b}^{n}$ des Fahrzeugs bezüglich des Navigationskoordinatensystems. Mit den Elementen dieser Rotationsmatrix läßt sich die Orientierung des Fahrzeugs in den Euler-Winkeln:

\footnotetext{
${ }^{4}$ IMU: Inertial Measurement Unit
} 


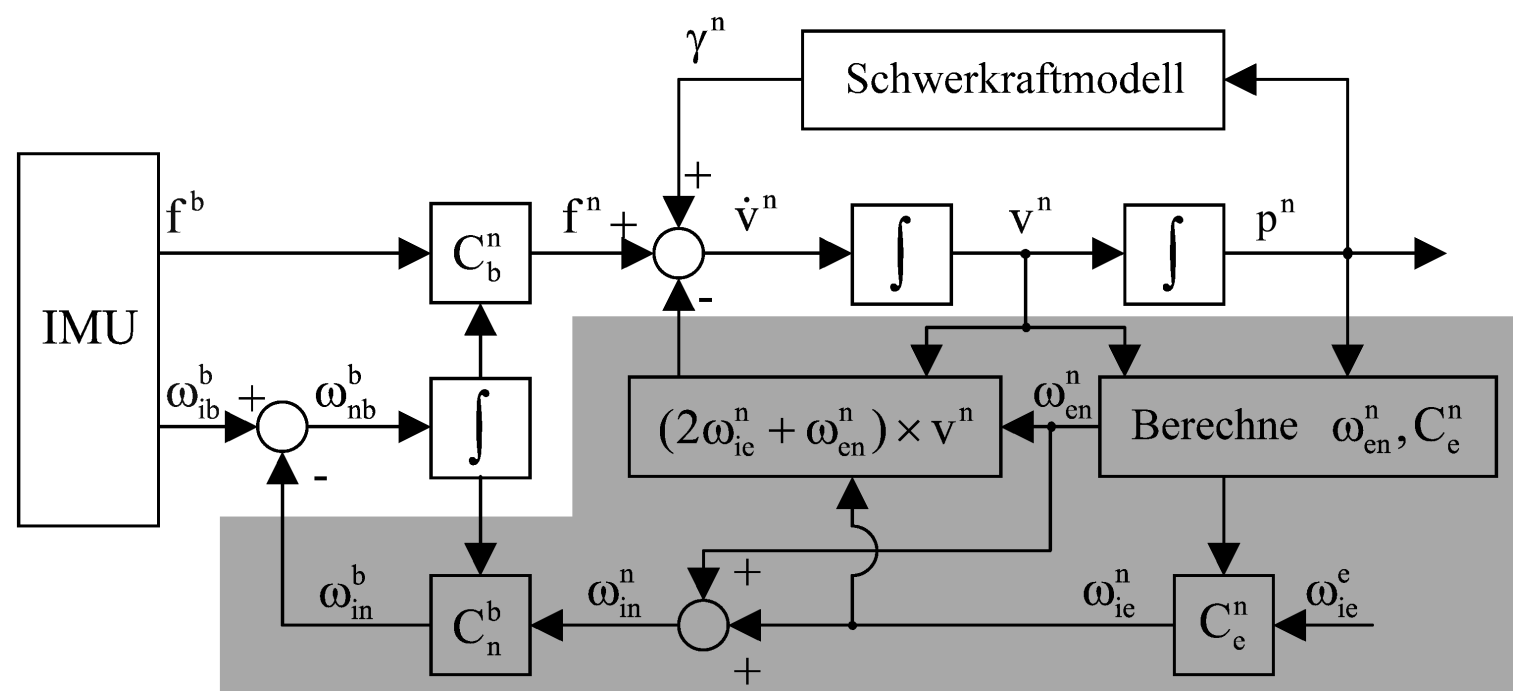

Abbildung 3.7: Blockschaltbild der Strapdown-Rechnung [29] zur Bestimmung von Position $\mathbf{p}^{n}$, Geschwindigkeit $\mathbf{v}^{n}$, Beschleunigung $\dot{\mathbf{v}}^{n}$ und Orientierung $\mathbf{C}_{b}^{n}$ des Fahrzeugs. Grau hinterlegt sind die Rechenblöcke zur Berücksichtigung der Erdkrümmung und -drehung.

Wankwinkel $v$ (Drehung um die Fahrzeuglängsachse), Nickwinkel $\xi$ (Drehung um die Fahrzeugquerachse) und Gierwinkel $\psi$ (Drehung um die Fahrzeughochachse) des Fahrzeugs [4] ausdrücken:

$$
\begin{aligned}
\mathbf{C}_{b}^{n} & =\left[\begin{array}{lll}
c_{11} & c_{12} & c_{13} \\
c_{21} & c_{22} & c_{23} \\
c_{31} & c_{32} & c_{33}
\end{array}\right], \\
v & =\arctan 2\left(c_{32}, c_{33}\right), \\
\xi & =-\arctan \left(\frac{c_{31}}{\sqrt{1-c_{31}^{2}}}\right), \\
\psi & =\arctan 2\left(c_{21}, c_{11}\right) .
\end{aligned}
$$

Wie in Abbildung 3.7 dargestellt werden die in Körperkoordinaten $b$ gemessenen Beschleunigungen $\mathbf{f}^{b}$ mit $\mathbf{C}_{b}^{n}$ in Navigationskoordinaten $n$

$$
\mathbf{f}^{n}=\mathbf{C}_{b}^{n} \mathbf{f}^{b}
$$

transformiert. Da die seismische Masse der Beschleunigungssensoren sowohl auf Körperbeschleunigungen als auch auf die (negative) Erdbeschleunigung reagiert, muß die positionsabhängige Erdbeschleunigung $\gamma^{n}$ kompensiert werden [7]. Die gemessene Beschleunigung wird außerdem um Coriolisterme bereinigt, um die zeitliche Ableitung der Geschwindigkeit zu erhalten:

$$
\dot{\mathbf{v}}^{n}=\mathbf{C}_{b}^{n} \mathbf{f}^{b}-\left(2 \omega_{i e}^{n}+\omega_{e n}^{n}\right) \times \mathbf{v}^{n}+\gamma^{n} .
$$


Eine zeitliche Integration liefert die Geschwindigkeit $\mathbf{v}^{n}=\left(v_{N}, v_{E}, v_{D}\right)$, eine weitere zeitliche Integration mit darauffolgender Transformation in Ellipsoidkoordinaten liefert die Position $\mathbf{p}^{n}=(\lambda, \varphi, h)$ in Navigationskoordinaten $n$. Mit der Position und der Geschwindigkeit werden die Rotationsgeschwindigkeit $\omega_{e n}^{n}$ und Orientierung $\mathbf{C}_{e}^{n}$ des Navigationskoordinatensystems bezüglich des Erdkoordinatensystems und die Erddrehrate in Navigationskoordinaten $\omega_{i e}^{n}$ bestimmt. Deren in Körperkoordinaten $b$ transformierte Summe

$$
\omega_{i n}^{b}=\mathbf{C}_{n}^{b}\left(\omega_{i e}^{n}+\omega_{e n}^{n}\right)
$$

stellt die Drehrate des Navigationskoordinatensystems $n$ bezüglich des Inertialsystems $i$ dar und wird in (3.2) für die Korrektur der gemessenen Drehraten verwendet.

Die Bahnfolgeregelung benötigt die Lösung der in Abbildung 3.7 dargestellten Strapdown-Rechnung in Echtzeit. Dazu muß deren Berechnung für jeden Abtastschritt der Inertialsensoren durchgeführt werden. Die Details der robusten numerischen Realisierung sind in der Diplomarbeit des Autors [14] beschrieben. Für eine praxistaugliche Anwendung müssen die herstellerspezifischen Eigenschaften des Inertialsensorpaketes und dessen interne Aufbereitung der Sensordaten bei der Integration berücksichtigt sowie eine numerisch stabile Integration der Winkelgeschwindigkeiten über eine Quaternionenrechnung durchgeführt werden.

\subsubsection{Anfangsausrichtung}

Für die Integration der Drehrate $\omega_{n b}^{b}$, der Beschleunigung $\dot{\mathbf{v}}^{n}$ und der Geschwindigkeit $\mathbf{v}^{n}$ in Abbildung 3.7 werden jeweils Anfangswerte benötigt. Diese sind nach dem Einschalten des Systems zunächt nicht bekannt und sollen ohne Zutun des Fahrers ermittelt werden. Die Anfangsposition $\mathbf{p}_{0}^{n}$ wird in ausreichender Genauigkeit über ein Satellitennavigationssystem ermittelt. Bei Fahrzeugstillstand ist die Geschwindigkeit $\mathbf{v}_{0}^{n}$ bezüglich der Erdoberfläche null. Für den Fahrzeugstillstand in der Initialisierungsphase hat dabei der Fahrer zu sorgen. Der dritte Satz von Anfangswerden, der benötigt wird, ist die Anfangsorientierung $\mathbf{C}_{0_{n}}^{b}$ des Fahrzeugs im Raum. Die erwartete Messung der Beschleunigungssensoren $\tilde{\mathbf{f}}^{b}$ beträgt für eine zu ermittelnde Anfangsorientierung $\hat{\mathbf{C}}_{0_{n}}{ }^{2}$ im stationären (unbeschleunigten) Fall

$$
\tilde{\mathbf{f}}^{b}=-\hat{\mathbf{C}}_{0_{n}}^{b} \gamma^{n}
$$

da die negative Erdbeschleunigung $\gamma^{n}$ bei Fahrzeugstillstand die einzige gemessene Beschleunigung ist. Dieser Ausdruck läßt sich in den anfänglichen Wank- und Nickwinkel umformen [7]. Die Orientierung in der Ebene senkrecht zu dem Vektor der Erdbeschleunigung läßt sich nicht über die Beschleunigungsmessung ermitteln. Damit auch diese Komponente und damit die komplette Anfangsausrichtung in Form der Rotationsmatrix $\mathbf{C}_{0 n}^{b}$ bestimmt werden kann, muß zusätzlich zu der Beschleunigungsmessung $\tilde{\mathbf{f}}^{b}$ die Messung der Drehratensensoren $\tilde{\boldsymbol{\omega}}_{i b}^{b}$ herangezogen werden. Bei bekanntem Breitengrad $\lambda$ 
der Position läßt sich die geschätzte Anfangsausrichtung $\hat{\mathbf{C}}_{0_{n}}^{b}$ über den genau bekannten Betrag der Erddrehrate $\omega_{i e}$ bestimmen. Voraussetzung dafür ist, daß die Sensoren die Erddrehrate ausreichend genau auflösen. Nach der Herleitung in [7] gilt:

$$
\hat{\mathbf{C}}_{0_{n}}^{b}=\left[\tilde{\mathbf{f}}^{b}, \tilde{\boldsymbol{\omega}}_{i b}^{b}, \tilde{\mathbf{f}}^{b} \times \tilde{\boldsymbol{\omega}}_{i b}^{b}\right]\left[\begin{array}{ccc}
\frac{1}{\gamma^{n}} \tan (\lambda) & 0 & \frac{1}{\gamma^{n}} \\
\frac{1}{\omega_{i e}^{n} \cos (\lambda)} & 0 & 0 \\
0 & \frac{1}{\omega_{i e} \gamma^{n} \cos (\lambda)} & 0
\end{array}\right]
$$

Die Messung der Erddrehrate für eine ausreichend genaue Anfangsausrichtung benötigt bei den verwendeten faseroptischen Drehratensensoren eine Mittelwertbildung über einige Minuten. Sollte diese Wartezeit für den Fahrer nicht akzeptabel sein, kann der anfängliche Gierwinkel auch anderweitig ermittelt werden. Zu den alternativen Methoden zählen zum Beispiel die satellitenbasierte Lagewinkelmessung über Mehrantennensysteme [2] oder die manuelle Eingabe der groben Anfangsorientierung des Fahrzeugs an einer bekannten Referenzposition des Prüfgeländes.

\subsection{Integriertes Navigationssystem}

Die in Abschnitt 3.1.2 beschriebene und in Abbildung 3.7 skizzierte inertiale Navigationsrechnung liefert die Position, Geschwindigkeit und Ausrichtung des Fahrzeugs bezüglich des Navigationskoordinatensystems. Sie zeichnet sich durch eine hohe Datenrate und gute relative Genauigkeit bei hoher Verfügbarkeit aus, da das gekapselte Inertialsensorpaket nahezu unabhängig von Störungen aus seiner Umgebung ist. Die rein inertiale Navigation hat jedoch den Nachteil, daß sich kleine Fehler in den Anfangswerten durch schnell veränderliche (aber beschränkte) Fehlerterme der Navigationslösung bemerkbar machen [7]. Exemplarisch ist in Abbildung 3.8 die Fehlerentwicklung bei einem anfänglichen Gierwinkelfehler von einem Grad dargestellt.

Auch bei einer perfekten Initialisierung führen Sensorfehler mit der Zeit zwangsläufig zu einer Falschausrichtung und damit zu einem schnell anwachsenden Fehler. Dieser würde in der Praxis eine regelmäßige Neuausrichtung und Initialisierung vor jedem Fahrversuch notwendig machen. Um dies zu vermeiden und um ein langzeitstabiles Navigationssystem zu erhalten, müssen die Fehler der Strapdown-Rechnung regelmäßig ermittelt und beseitigt werden. Dies geschieht durch den Einsatz eines Kalman-Filters. Die in Folge beschriebene Methode ist die klassische und am weitesten verbreitete mehrerer möglicher Varianten zur Kombination von Inertial- und Satellitennavigation [36]. Bei den neueren Varianten wird die Datenauswertung des Satellitennavigationssystems und des Inertialsensorpakets in das Fusionsfilter verschoben. Als weitere Variation kann anstatt des im klassischen Ansatz verwendeten geodätischen Koordinatensystems ein erdmittelpunktfestes Koordinatensystem verwendet werden. Hier wird in Abwägung des Entwicklungs- 


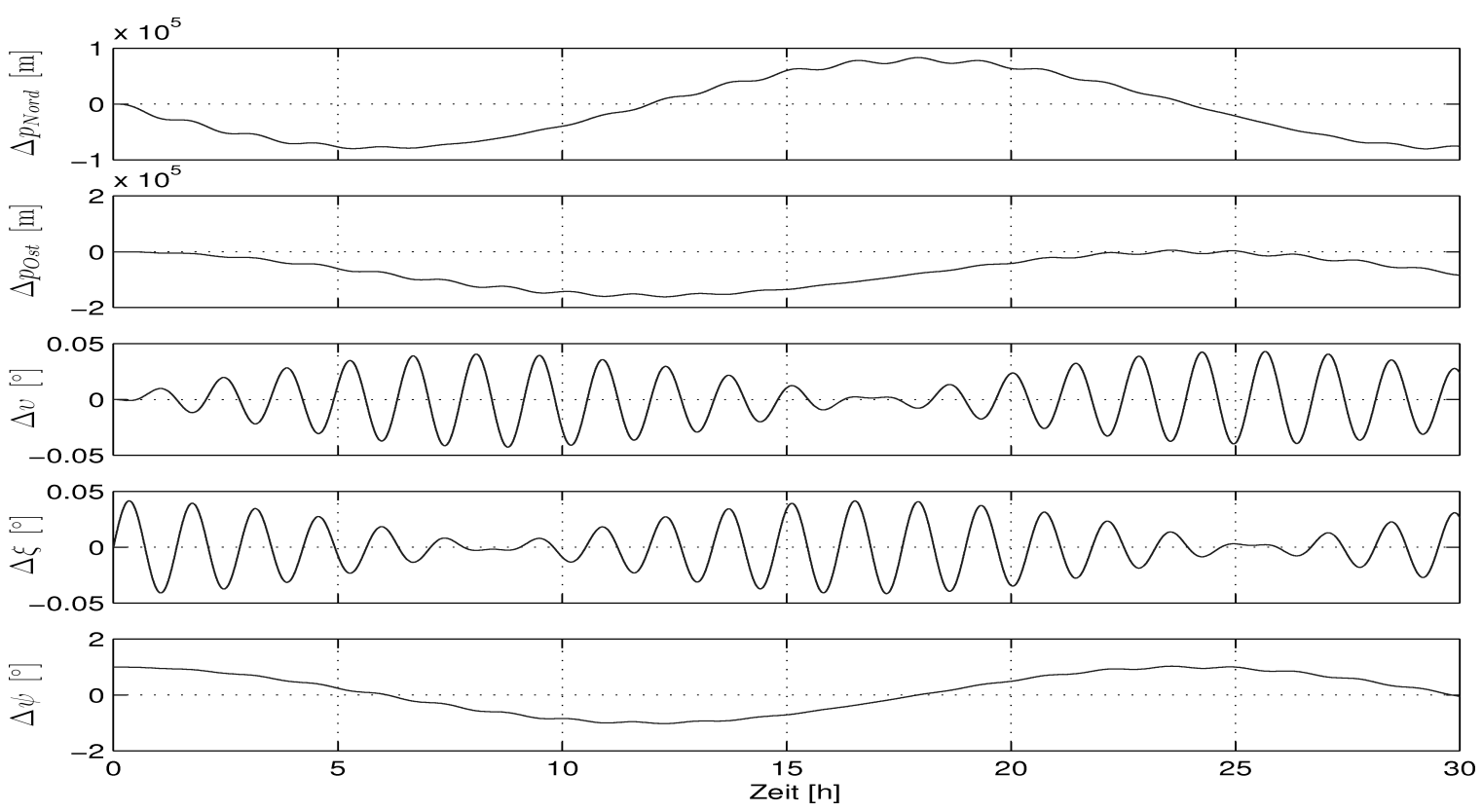

Abbildung 3.8: Exemplarische Entwicklung der Positionsfehler $\left(\Delta p_{N o r d}, \Delta p_{O s t}\right)$ und der Orientierungsfehler $(\Delta v, \Delta \xi, \Delta \psi)$ der ungestützten inertialen Navigationsrechnung über der Zeit bei einem anfänglichen Gierwinkelfehler $\Delta \psi$ von einem Grad [7].

und Rechenzeitbedarfs gegen die bei den neuen Varianten erreichbare bessere Stabilität des Navigationssystems der klassische Ansatz gewählt. Für die Ermittlung des Navigationsfehlers durch das Fusionsfilter wird das im folgenden Abschnitt beschriebene Fehlermodell der Strapdown-Rechnung benötigt.

\subsubsection{Fehlermodell der Strapdown-Rechnung}

Der Fehlerzustand

$$
\delta \mathbf{x}=\left(\delta \mathbf{p}^{T}, \delta \mathbf{v}^{T}, \delta \boldsymbol{\rho}^{T}\right)^{T}
$$

des inertialen Navigationssystems ist definiert als Differenz $\delta \mathbf{x}=\mathbf{x}-\hat{\mathbf{x}}$ der tatsächlichen und der mittels Strapdown-Rechnung bestimmten Werte. Der Fehlervektor $\delta \mathbf{x}$ umfaßt den Positionsfehler $\delta \mathbf{p}=(\delta \lambda, \delta \varphi, \delta h)^{T}$, den Geschwindigkeitsfehler $\delta \mathbf{v}=\left(\delta v_{N}, \delta v_{E}, \delta v_{D}\right)^{T}$ und den Orientierungsfehler $\delta \boldsymbol{\rho}=\left(\delta \epsilon_{x}, \delta \epsilon_{y}, \delta \epsilon_{z}\right)^{T}$.

Die Differentialgleichung des Fehlermodells lautet [7, 14]:

$$
\dot{\delta}=\mathbf{F} \delta \mathbf{x}+\mathbf{G u}
$$

mit der Dynamikmatrix F in Abhängigkeit von dem Inertialsensorrauschen $\mathbf{u}$. Die Ver- 
teilungsmatrix

$$
\mathbf{G}=\left[\begin{array}{cc}
\mathbf{0} & \mathbf{0} \\
\mathbf{C}_{b}^{n} & \mathbf{0} \\
\mathbf{0} & \mathbf{C}_{b}^{n}
\end{array}\right]
$$

beschreibt, wie sich das Rauschen $\mathbf{u}$ auf den Fehlerzustand $\delta \mathbf{x}$ auswirkt. Die einzelnen Elemente der Matrix F werden in [7] hergeleitet und sind im Anhang aufgeführt. Eine vereinfachte Form der Dynamikmatrix F vernachlässigt den Einfluß der Schuler- und Coriolis-Terme [21, 34] und verwendet für die Darstellung der Position anstatt der Ellipsoidkoordinaten ein lokales kartesisches Koordinatensystem. Sie besitzt die Form

$$
\mathbf{F}=\left[\begin{array}{ccc}
\mathbf{0}_{3} & \mathbf{I}_{3} & \mathbf{0}_{3} \\
\mathbf{0}_{3} & \mathbf{0}_{3} & \mathbf{F}_{v \rho} \\
\mathbf{0}_{3} & \mathbf{0}_{3} & \mathbf{F}_{\rho \rho}
\end{array}\right]=\left[\begin{array}{ccc|ccc|ccc}
0 & 0 & 0 & 1 & 0 & 0 & 0 & 0 & 0 \\
0 & 0 & 0 & 0 & 1 & 0 & 0 & 0 & 0 \\
0 & 0 & 0 & 0 & 0 & 1 & 0 & 0 & 0 \\
\hline 0 & 0 & 0 & 0 & 0 & 0 & 0 & f_{D} & -f_{E} \\
0 & 0 & 0 & 0 & 0 & 0 & -f_{D} & 0 & f_{N} \\
0 & 0 & 0 & 0 & 0 & 0 & f_{E} & -f_{N} & 0 \\
\hline 0 & 0 & 0 & 0 & 0 & 0 & 0 & \omega_{D} & -\omega_{E} \\
0 & 0 & 0 & 0 & 0 & 0 & -\omega_{D} & 0 & \omega_{N} \\
0 & 0 & 0 & 0 & 0 & 0 & \omega_{E} & -\omega_{N} & 0
\end{array}\right]
$$

Die drei Elemente $\left(f_{N}, f_{E}, f_{D}\right)$ von $\mathbf{F}_{v \rho}$ repräsentieren die Beschleunigung, die die seismische Masse der Beschleunigungssensoren erfährt. Die Elemente $\left(\omega_{N}, \omega_{E}, \omega_{D}\right)$ von $\mathbf{F}_{\rho \rho}$ repräsentieren die Drehgeschwindigkeit

$$
\boldsymbol{\omega}_{i n}^{n}=\left[\begin{array}{c}
\omega_{N} \\
\omega_{E} \\
\omega_{D}
\end{array}\right]=\left[\begin{array}{c}
\omega_{e} \cos (\lambda) \\
0 \\
-\omega_{e} \sin (\lambda)
\end{array}\right]+\left[\begin{array}{c}
\frac{v_{E}}{R_{\varphi}+h} \\
\frac{-v_{N}}{R_{\lambda}+h} \\
\frac{-v_{E} \tan (\lambda)}{R_{\varphi}+h}
\end{array}\right]
$$

des Navigationskoordinatensystems $n$ bezüglich des Inertialsystems $i$. Der erste Summand repräsentiert die Erddrehung, der zweite Summand die sogenannte „Transportrate“, die bei Geschwindigkeiten, wie sie bei Fahrzeugen üblich sind, gegenüber der Erddrehung klein ist, da $R_{\varphi}$ und $R_{\lambda}$ von der Größenordnung des Erdradius sind.

Die zu (3.13) gehörende Messgleichung mit der Messmatrix $\mathbf{H}$ und dem Messrauschen w lautet:

$$
\mathbf{z}=\mathbf{H} \delta \mathbf{x}+\mathbf{w}
$$

Für die Schätzung des Fehlerzustandes $\delta$ x mit Hilfe des Modells (3.13)-(3.17) zur regelmäßigen Korrektur der Navigationsrechnung muß dessen Beobachtbarkeit sichergestellt sein. 


\subsubsection{Beobachtbarkeit des Fehlerzustandes}

Für die Beobachtbarkeitsanalyse [8] des Fehlermodells (3.13)-(3.17) wird die vereinfachte Dynamikmatrix (3.15) verwendet. Eine detaillierte Betrachtung der Beobachtbarkeit für verschiedene Stützkonfigurationen ist in [36, 3] zu finden. An den drei Nullspalten der Dynamikmatrix (3.15) ist zu erkennen, daß für die Beobachtbarkeit des Positionsfehlers $\delta \mathbf{p}$ eine Positionsmessung benötigt wird. Bei einer dreidimensionalen Positionsmessung, wie sie mit Satellitennavigationsempfängern vorgenommen werden kann, ist die Messmatrix $\mathbf{H}$ in (3.17) gegeben als

$$
\mathbf{H}=\left(\mathbf{I}_{3}, \mathbf{0}_{3}, \mathbf{0}_{3}\right)
$$

Die ersten vier Blockzeilen der Kalman-Beobachtbarkeitsmatrix lauten:

$$
\boldsymbol{\Gamma}=\left[\begin{array}{c}
\mathbf{H} \\
\mathbf{H F} \\
\mathbf{H F}^{2} \\
\mathbf{H F}^{3}
\end{array}\right]=\left[\begin{array}{ccc}
\mathbf{I}_{3} & \mathbf{0}_{3} & \mathbf{0}_{3} \\
\mathbf{0}_{3} & \mathbf{I}_{3} & \mathbf{0}_{3} \\
\mathbf{0}_{3} & \mathbf{0}_{3} & \mathbf{F}_{v \rho} \\
\mathbf{0}_{3} & \mathbf{0}_{3} & \mathbf{F}_{v \rho} \mathbf{F}_{\rho \rho}
\end{array}\right]
$$

Daran erkennt man, daß für $\mathbf{F}_{v \rho}=\mathbf{0}$, das heißt für $f_{N}=f_{E}=f_{D}=0$, das System nicht beobachtbar ist, da $\operatorname{Rang}(\boldsymbol{\Gamma})=6$. Dieser Fall wird in der Praxis aber kaum vorkommen, da zumindest für landgebundene Fahrzeuge die gemessene Beschleunigungskomponente $f_{D}$ durch die Erdanziehungskraft ungleich null ist. Für $\left(f_{N}, f_{E}, f_{D}\right)^{T} \neq \mathbf{0}_{3}$ ist $\operatorname{Rang}(\boldsymbol{\Gamma})=8$, wenn $\boldsymbol{\omega}_{i n}^{n}$ aus (3.16) zu klein und nicht messbar ist (Der Rang der schiefsymmetrischen Matrix $\mathbf{F}_{v \rho}$ ist 2). Der in diesem Fall nicht beobachtbare Zustand ist der Orientierungswinkel um den aktuell registrierten Beschleunigungsvektor $\mathbf{f}_{b}$ [3]. Laut (3.15) und (3.16) ist die vierte Blockzeile $\left(\mathbf{0}_{3}, \mathbf{0}_{3}, \mathbf{F}_{v \rho} \mathbf{F}_{\rho \rho}\right)$ der Beobachtbarkeitsmatrix (3.19) erst durch Detektion der Erddrehrate $\omega_{e}$ oder mit ausreichend großer Geschwindigkeit $\mathbf{v}^{n}$ ungleich null. Dann ist $\operatorname{Rang}(\boldsymbol{\Gamma})=9$ und das System vollständig beobachtbar.

Bei Fahrzeugstillstand oder bei beschleunigungsarmer Fahrt dominiert die Erdbeschleunigung den gemessenen Beschleunigungsvektor. Der Gierwinkel als Winkel der Drehung um die Hochachse ist daher schlecht beobachtbar. Dessen Beobachtbarkeit läßt sich durch quer- und längsbeschleunigungsreiches Fahren, also möglichst starke und häufige Änderung der Beschleunigungsrichtung, verbessern. Für die Praxis bedeutet das Ergebnis der Beobachtbarkeitsanalyse, daß die verwendeten Drehratensensoren die Erddrehrate ausreichend genau aufösen müssen, um in jeder Situation die Beobachtbarkeit des Fehlermodells (3.13)-(3.17) und damit eine langzeitstabile Lösung der Navigationsrechnung zu gewährleisten. 
Messung der Fahrzeugbewegung

\subsubsection{Stützung der Navigationsrechnung mittels Differential- GPS}

Wie im letzten Abschnitt gezeigt ist es prinzipiell möglich, den Fehlerzustand der Strapdown-Rechnung mit Hilfe von Positionsmessungen zu ermitteln. Eine Möglichkeit, die sich zur Bestimmung der drei Positionskomponenten des Fahrzeugs auf dem Prüfgelände besonders anbietet, ist die Satellitennavigation über Differential-GPS [20, 12]: Die Bestimmung der absoluten Position über GPS ist langzeitstabil (aber nur mit geringer Datenrate verfügbar). Trotz der guten Empfangsbedingungen auf dem Prüfgelände kann erstens ein ungestörter Empfang aber nicht immer garantiert werden, die Genauigkeit der satellitenbasierten Positionsbestimmung ist u.a. abhängig von den Umgebungsbedingungen. Die situationsabhängige Genauigkeit wird über das Empfängerfilter gegebenenfalls aber einbezogen und kann somit bei der Sensorfusion in bestimmten Grenzen berücksichtigt werden.

Das Satellitennavigationssystem basiert zweitens auf einer Laufzeitmessung der von Satelliten ausgestrahlten Signalen. Die Signale müssen die Erdatmosphäre durchlaufen und sind dabei unbekannten Laufzeitverschiebungen unterworfen. Bei Differential-GPS-Messungen ist es mit Hilfe einer ortsfesten Basisstation dann möglich, Dezimetergenauigkeit der gemessenen Position zu erreichen. Eine solche Basisstation besteht aus einem stationären GPS-Empfänger, der mit Hilfe seiner bekannten Antennenposition Korrekturwerte für die gemessenen Satellitenentfernungen berechnet. Diese Korrekturwerte werden über eine Funkstrecke an den bewegten Empfänger gesendet.

In einem satellitengestützten inertialen Navigationssystem werden weiter jeweils die positiven Eigenschaften von inertialer und satellitengestützter Navigation vereint, indem die Positionsmessung des Satellitennavigationsempfängers dazu genutzt wird, den aktuellen Fehler der inertialen Navigationsrechnung über einen Beobachter zu schätzen und dadurch zu beseitigen. Diese Systematik wird auch als „Stützung“ der Navigationsrechnung bezeichnet. In der Gesamtheit wird so eine dynamische und hochpräzise Ermittlung der Position $\hat{\mathbf{p}}$, Geschwindigkeit $\hat{\mathbf{v}}$ und Beschleunigung â des Fahrzeugschwerpunktes sowie der Orientierung $\hat{\rho}$ und Drehrate $\hat{\omega}$ des Fahrzeugs erreicht.

Das für die Bahnfolgeregelung realisierte integrierte Navigationssystem ist in der sogenannten „Feedback“-Konfiguration ausgeführt (Abbildung 3.9). Die verwendeten Sensoren sind ein inertiales Sensorpaket (IMU) und ein Differential-GPS-Empfänger (DGPS). Die Messdaten der Inertialsensoren werden in der Strapdown-Rechnung (INS) - wie in Abschnitt 3.1.2 beschrieben - verarbeitet. Der DGPS-Empfänger liefert als Messergebnis die Position $\tilde{\mathbf{p}}$. In der Feedback-Konfiguration wird die Differenz der durch die Strapdown-Rechnung berechneten Position $\hat{\mathbf{p}}$ und der vom DGPS-Empfänger gemessenen Position $\tilde{\mathbf{p}}$ gebildet. Dies ermöglicht es, mit dem Fehlermodell der Navigationsrechnung über ein erweitertes Kalman-Filter (EKF) die Fehler $\delta \hat{\mathbf{x}}$ der Navigationsrechnung 


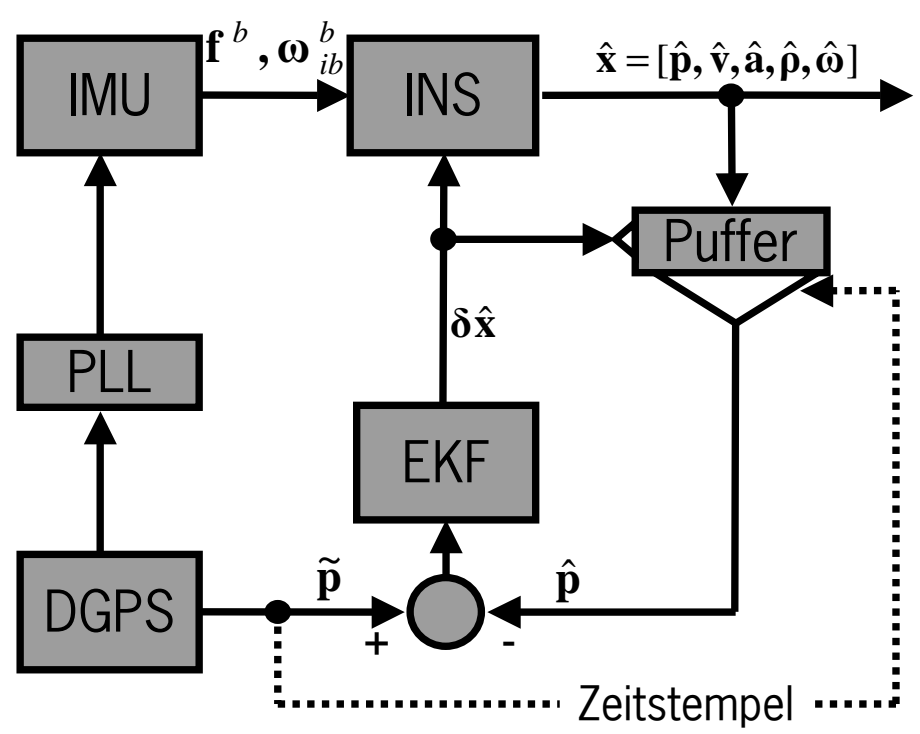

Abbildung 3.9: Struktur des integrierten Navigationssystems mit dem inertialen Navigationssystem aus Sensorpaket (IMU) und inertialer Navigationsrechnung (INS), dem erweiterten Kalman-Filter (EKF) und Differential-GPS (DGPS) zur Korrektur des Navigationsfehlers $\delta$ x sowie Sensorsynchronisierung durch eine "phase-locked-loop“ (PLL).

zu schätzen und zu korrigieren. Das Ergebnis der Strapdown-Rechnung steht dabei wie auch bei der ungestützten Navigationsrechnung ohne Verzögerung zur Verfügung.

Da das Phasenzentrum der GPS-Antenne nicht im Zentrum des Inertialsensorspakets liegen kann, sondern über einen in Körperkoordinaten bekannten Verschiebungsvektor davon entfernt ist, wird vor Bildung der Positiondifferenz die gemessene Position des Phasenzentrums der Antenne mit Hilfe des Verschiebungsvektors auf die Position Inertialsensorpakets transformiert.

Das Ergebnis der Navigationsrechnung liegt zunächst für die Position des Inertialsensorpakets vor. Da der Regler die Messungen im Schwerpunkt erwartet, werden Position und Geschwindigkeiten schließlich in den Schwerpunkt des Fahrzeugs transformiert. Die Position wird dabei außerdem in das lokale ebene Koordinatensystem $t$ mit Ursprung im Zentrum des Prüfgeländes umgerechnet. Gleichzeitig wird aus den verfügbaren Daten der Schwimmwinkel (s.u.) berechnet.

In dem DGPS-Empfänger finden umfangreiche Berechnungen statt, deren Ergebnis in Form der Antennenposition und deren geschätzten Standardabweichungen über eine serielle Schnittstelle an das Navigationssystem übergeben wird. Durch die Berechnung und serielle Übertragung entsteht eine Differenz zwischen dem Zeitpunkt der Messung und der Verfügbarkeit der Ergebnisse von bis zu einer halben Sekunde. Eine neu eingetroffene DGPS-Messung darf daher nicht mit dem aktuellen Ergebnis der Strapdown-Rechnung zum Zwecke der Fehlerschätzung verglichen werden, sondern muß über eine geeignete 
Synchronisierung dem Datensatz der Navigationsrechnung zugeordnet werden, der zum Messzeitpunkt des DGPS-Empfängers berechnet wurde. Diese Synchronisierung geschieht wie in Abbildung 3.9 gezeigt über einen Ergebnispuffer und den Zeitstempel der DGPSMessung.

\subsubsection{Synchronisierung der Sensoren}

Um sicherzustellen, dass zu jeder Messung des DGPS genau eine Messung des Inertialsensors (IMU) mit einem exakt gleichen Abtastzeitpunkt bereitsteht, wird die Abtastung des Inertialsensorpakets (IMU) wie in Abbildung 3.9 dargestellt über eine „phase locked loop (PLL) mit dem Sekundensignal des DGPS synchronisiert. Jedes Ergebnis der inertialen Navigationsrechnung (INS) trägt ebenso wie die Lösungen des DGPS einen Zeitstempel. Trifft ein Ergebnis des DGPS ein, werden anhand des Zeitstempels das zugehörige Ergebnis der Navigationsrechnung aus einem Ringpuffer entnommen und die darin enthaltene Position mit der gemessenen Position des DGPS verglichen. Zuvor wird die Position des Phasenzentrums der GPS-Antenne mit Hilfe der bekannten Fahrzeugorientierung und des Verschiebungsvektors auf die Position des Inertialsensors transformiert.

Das inertiale Sensorpaket arbeitet mit einer Datenrate von 400Hz, der GPS-Empfänger mit bis zu 20Hz. Hat das Kalman-Filter die Korrektur vorgenommen, müßte die Strapdown-Rechnung von dem Gültigkeitszeitpunkt der Korrektur an mit den gespeicherten Rohdaten des Inertialsensors wiederholt werden. Da die Verzögerung des Eintreffens der GPS-Messung aber bis zu einer halben Sekunde betragen kann, würden die zu den 400 Strapdown-Rechenschritten pro Sekunde zusätzlich hinzukommenden Schritte der Strapdown-Rechnung die maximal verfügbare Rechenleistung übersteigen. Um dies zu vermeiden, wird die Korrektur vom Gültigkeitszeitpunkt an für alle Ergebnisdatensätze im Puffer durchgeführt. Dies entspricht einer Parallelverschiebung aller nach dem Gültigkeitszeitpunkt der Korrektur bereits berechneten Navigationslösungen. Die Strapdown-Rechnung selbst wird für die Korrektur nicht wiederholt.

Durch diese Vereinfachung ergeben sich Fehler entsprechend der Fehlerdynamik, die sich beim Vergleich der exakten und der vereinfachten Berechnungsmethode an realen Messdaten mit künstlich um eine halbe Sekunde verzögerten GPS-Messungen im Bereich von einem Zentimeter bewegen [11].

\subsubsection{Erweitertes Kalman-Filter}

Das erweiterte Kalman-Filter hat gemäß Abbildung 3.9 die Aufgabe, aus den Unterschieden der Position $(\tilde{\mathbf{p}}-\hat{\mathbf{p}})$ aus der Navigationsrechnung $\hat{\mathbf{p}}$ und dem DGPS-Empfänger $\tilde{\mathbf{p}}$ anhand des Fehlermodells (3.13)-(3.17) den Fehlerzustand $\delta \hat{\mathbf{x}}$ der Strapdown-Rechnung zu 
ermitteln. Die Zustände der Navigationsrechnung werden daraufhin um den geschätzten Fehler $\delta \hat{\mathbf{x}}$ korrigiert, um ein lanzeitstabiles Navigationsergebnis zu erhalten.

Die folgende Beschreibung des Kalman-Filters ist eine Zusammenfassung aus der Diplomarbeit [14] des Autors. Das Fehlermodell wird durch die Differentialgleichung (3.13) und die Messgleichung (3.17) beschrieben. In diesen Gleichungen ist w das Messrauschen und $\mathbf{u}$ das als weiß angenommene Systemrauschen

$$
\mathbf{u}=\left[\begin{array}{c}
\delta \mathbf{f}^{b} \\
\delta \boldsymbol{\omega}_{i b}^{b}
\end{array}\right]
$$

mit der Kovarianzmatrix

$$
\mathbf{E}\left\langle\mathbf{u}(t) \mathbf{u}^{T}(\tau)\right\rangle=\mathbf{Q} \delta(t-\tau)
$$

und der Diagonalmatrix

$$
\mathbf{Q}=\operatorname{diag}\left(\sigma_{f_{x}}^{2}, \sigma_{f_{y}}^{2}, \sigma_{f_{z}}^{2}, \sigma_{\omega x}^{2}, \sigma_{\omega y}^{2}, \sigma_{\omega z}^{2}\right) .
$$

Das Symbol $\delta(t-\tau)$ bezeichnet die Dirac-Funktion, $\sigma_{f}$ und $\sigma_{\omega}$ sind die Standardabweichungen der Beschleunigungssensoren und Drehratensensoren. Die zeitdiskrete Form der Gleichungen (3.13) und (3.17) lautet

$$
\begin{aligned}
\delta \mathbf{x}_{k+1} & =\boldsymbol{\Phi}_{k} \delta \mathbf{x}_{k}+\mathbf{u}_{k} \\
\mathbf{z}_{k} & =\mathbf{H} \delta \mathbf{x}_{k}+\mathbf{w}_{k}
\end{aligned}
$$

Hier sind $\delta \mathbf{x}_{k}$ der Zustand zum Zeitpunkt $t_{k}, k \in \mathbb{N}, \boldsymbol{\Phi}_{k}$ die zeitdiskrete Dynamikmatrix, um $\delta \mathbf{x}_{k+1}$ aus $\delta \mathbf{x}_{k}$ zu bestimmen, $\mathbf{z}_{k}$ eine Messung zum Zeitpunkt $t_{k}$ und $\mathbf{H}$ die Messmatrix.

Die Kovarianzmatrizen für die Rauschterme $\mathbf{u}_{k}$ und $\mathbf{w}_{k}$ sind

$$
\begin{aligned}
\mathbf{E}\left\langle\mathbf{u}_{h} \mathbf{u}_{i}^{T}\right\rangle & =\delta_{i h} \mathbf{Q}_{h i} \\
\mathbf{E}\left\langle\mathbf{w}_{h} \mathbf{w}_{i}^{T}\right\rangle & =\delta_{i h} \mathbf{R}_{h i} \\
\mathbf{E}\left\langle\mathbf{u}_{h} \mathbf{w}_{i}^{T}\right\rangle & =\mathbf{0}
\end{aligned}
$$

wobei $\delta_{i j}$ das Kronecker-Symbol ist.

Die zeitdiskrete Dynamikmatrix $\boldsymbol{\Phi}_{k}$ wird für das Zeitintervall $\Delta t=t_{k+1}-t_{k}$ eines Abtastzyklus des DGPS-Empfängers durch die Matrixexponentialfunktion

$$
\mathbf{\Phi}_{k}=e^{\mathbf{F} \Delta t} \approx \mathbf{I}+\mathbf{F} \Delta t+\frac{(\mathbf{F} \Delta t)^{2}}{2 !}+\frac{(\mathbf{F} \Delta t)^{3}}{3 !}+\ldots
$$

berechnet. Die Gleichung für die zeitdiskrete Kovarianzmatrix [7]

$$
\begin{aligned}
\mathbf{Q}_{k} & =\mathbf{E}\left\langle\mathbf{u}_{k} \mathbf{u}_{k}^{T}\right\rangle \\
& =\int_{t_{k}}^{t_{k+1}} \int_{t_{k}}^{t_{k+1}} e^{\mathbf{F}\left(t_{k+1}-t\right)} \mathbf{G}(t) \mathbf{E}\left\langle\mathbf{u}(t) \mathbf{u}^{T}(\tau)\right\rangle \mathbf{G}^{T}(\tau)\left[e^{\mathbf{F}\left(t_{k+1}-\tau\right)}\right]^{T} \mathrm{~d} \tau \mathrm{d} t \\
& =\int_{t_{k}}^{t_{k+1}} e^{\mathbf{F}\left(t_{k+1}-t\right)} \mathbf{G}(t) \mathbf{Q}(t) \mathbf{G}^{T}(t)\left[e^{\mathbf{F}\left(t_{k+1}-t\right)}\right]^{T} \mathrm{~d} t
\end{aligned}
$$


kann durch folgende Approximationen mit der Verteilungsmatrix G (3.14) und der zeitdiskreten Dynamikmatrix $\boldsymbol{\Phi}_{k}$ (3.28) numerisch berechnet werden [21]:

$$
\mathbf{Q}_{k} \approx \frac{1}{2}\left[\boldsymbol{\Phi}_{k} \mathbf{G Q G}^{T} \boldsymbol{\Phi}_{k}^{T}+\mathbf{G Q G}^{T}\right] \Delta t .
$$

Die Elemente der Kovarianzmatrix $\mathbf{R}_{k}$ werden durch den DGPS-Empfänger zusammen mit der gemessenen Position bereitgestellt. Sie stammen aus dem empfängerinternen Filter und stellen eine Schätzung der Positionsgenauigkeit dar. Der Vektor $\mathbf{z}_{k}$ ist die Positionsdifferenz von Strapdown-Rechnung und DGPS-Messung zum Zeitpunkt $t_{k}$.

Nach der Initialisierung

$$
\begin{aligned}
\delta \hat{\mathbf{x}}_{0}^{-} & =\delta \overline{\mathbf{x}}_{0}=(0,0,0,0,0,0,0,0,0)^{T} \\
\mathbf{P}_{0}^{-} & =\operatorname{var}\left[\delta \hat{\mathbf{x}}_{0}^{-}\right]
\end{aligned}
$$

des Kalman-Filters wird mit jeder Messung $\mathbf{z}_{k}$ die Verstärkungsmatrix

$$
\mathbf{K}_{k}=\mathbf{P}_{k}^{-} \mathbf{H}_{k}^{T}\left[\mathbf{R}_{k}+\mathbf{H}_{k} \mathbf{P}_{k}^{-} \mathbf{H}_{k}^{T}\right]^{-1}
$$

und die Korrektur

$$
\delta \hat{\mathbf{x}}_{k}=\delta \hat{\mathbf{x}}_{k}^{-}+\mathbf{K}_{k}\left[\mathbf{z}_{k}-\mathbf{H}_{k} \delta \hat{\mathbf{x}}_{k}^{-}\right]
$$

sowie das Kovarianz-Update

$$
\mathbf{P}_{k}=\left[\mathbf{I}-\mathbf{K}_{k} \mathbf{H}_{k}\right] \mathbf{P}_{k}^{-}
$$

mit anschließendem Prädiktionsschritt

$$
\begin{aligned}
\delta \hat{\mathbf{x}}_{k+1}^{-} & =\boldsymbol{\Phi}_{k} \delta \hat{\mathbf{x}}_{k} \\
\mathbf{P}_{k+1}^{-} & =\boldsymbol{\Phi}_{k} \mathbf{P}_{k} \boldsymbol{\Phi}_{k}^{T}+\mathbf{Q}_{k}
\end{aligned}
$$

berechnet. Mit Hilfe des durch das Kalman-Filter geschätzten Fehlers

$$
\delta \hat{\mathbf{x}}=\left(\delta \hat{\mathbf{p}}^{T}, \delta \hat{\mathbf{v}}^{T}, \delta \hat{\boldsymbol{\rho}}^{T}\right)^{T}
$$

der Navigationsrechung wird deren Zustand $\hat{\mathbf{x}}$ korrigiert [14]. Durch die „Feedback“Konfiguration [7] des integrierten Navigationssystems bleibt der Fehler $\delta \mathbf{x}$ nahe null und die Linearisierung des Fehlermodells (3.13) ist über einen unbegrenzten Zeitraum gültig.

Als Folge der Korrektur des Zustandes $\hat{\mathbf{x}}$ der Navigationsrechnung ist das prädizierte $\delta \hat{\mathbf{x}}_{k}^{-}$des folgenden Schrittes der Filterberechnung gleich null und der Korrekturschritt des Kalman-Filters reduziert sich zu einer einfachen Gewichtung der gemessenen Differenzen durch die Kalman-Verstärkungsmatrix:

$$
\delta \hat{\mathbf{x}}_{k}=\mathbf{K}_{k} \mathbf{z}_{k}
$$




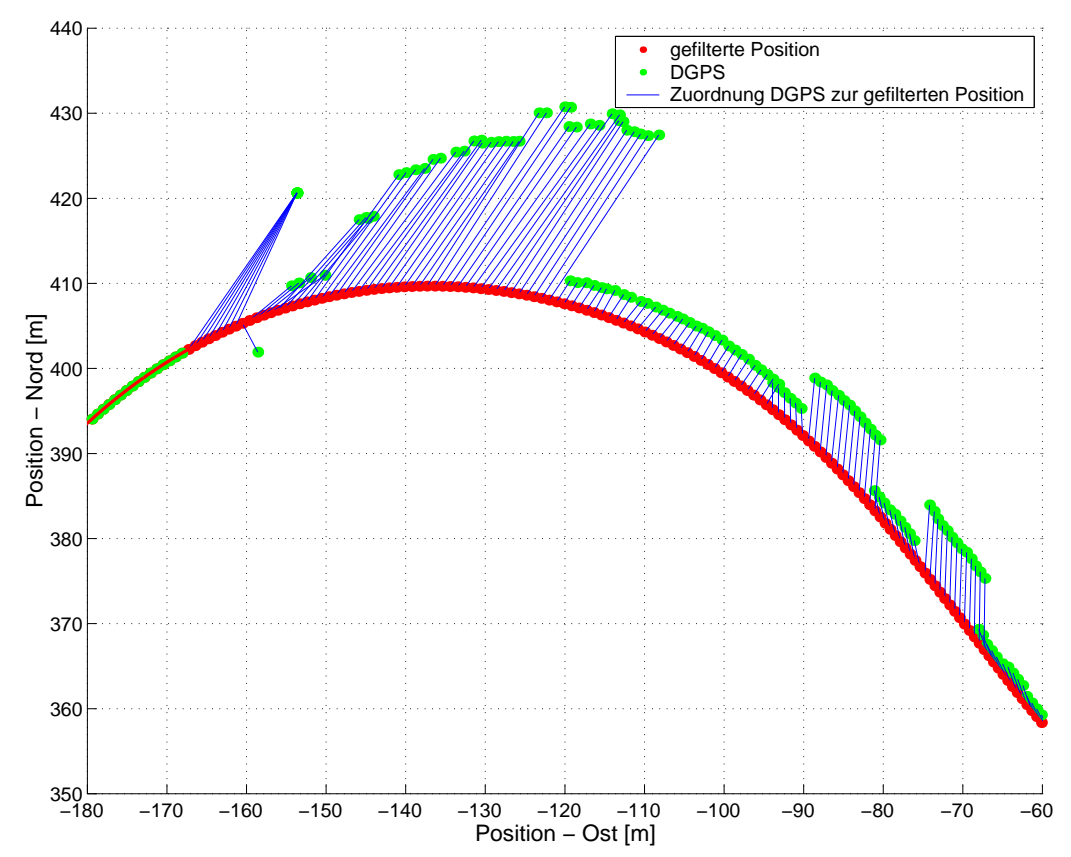

Abbildung 3.10: Stark korrelierte fehlerhafte DGPS-Positionsmessung nach einer Brückendurchfahrt im Vergleich zu der tatsächlichen gefilterten Position.

In der Realität stellen die Fehler der Positionsmessung über Differential-GPS kein weißes Rauschen dar, sondern sind zeitlich korreliert [36]. Ein Beispiel zeitlich korrelierter DGPS-Fehler zeigt Abbildung 3.10. Nach einer Brückendurchfahrt ist der Empfang kurzzeitig gestört. Die korrelierten Fehler der Messung kommen durch fehlerhaft geschätzte Entfernungen der Satelliten zustande. Die Annäherung an den tatsächlichen Wert erfolgt sprunghaft in Schrittweiten, die mit der Wellenlänge des von den Satelliten ausgesendeten Signals zusammenhängen. Die zeitliche Korrelation zweier aufeinander folgender Werte ist bei niedrigerer Abtastrate des GPS geringer, jedoch werden dadurch die Korrektursprünge in der Navigationslösung größer. Daher wird die maximale Abtastrate des GPS-Empfängers von $20 \mathrm{~Hz}$ genutzt, um den Betrag der Korrektursprünge möglichst gering zu halten.

Wenn sehr gute Empfangsbedingungen herrschen, wie es bei den meisten Prüfgeländen der Fall ist, und gleichzeitig zusätzliche Phasenmessungen des GPS-Trägersignals im GPS-Empfänger ausgewertet werden [12], ist der Fehler der absoluten Position im Ergebnis des integrierten Navigationssystems üblicherweise kleiner als $2 \mathrm{~cm}$. Dieser Fehler wird größer, wenn Reflektionen oder Abschattungen des GPS-Signals auftreten. Der Gierwinkel driftet auch bei längerem Fahrzeugstillstand nicht vom tatsächlichen Wert ab, da die faseroptischen Kreisel die Erddrehrate ausreichend genau aufösen. Der geschätzte maximale Fehler von Wank- und Nickwinkel beträgt ca. im betrachteten Fall (Kapitel 5) typischerweise $0.03^{\circ}$, während der geschätzte maximale Fehler des Gierwinkels bei ca. $0.3^{\circ}$ 
liegt (vgl. [36]). Diese Genauigkeit wird jedoch erst erreicht, wenn der Einschwingvorgang des Filters beendet ist. Wie bei der Beobachtbarkeitsanalyse in Abschnitt 3.2.2 gezeigt, wird der Einschwingvorgang stark verkürzt, wenn nach erfolgter Selbstausrichtung mit dem Fahrzeug einige wenige längs- und querbeschleunigungsreiche Manöver durchgeführt werden. Durch Einsatz des Kalman-Filters zur Stützung der Navigationsrechnung mittels Positionsmessungen wird so eine langzeitstabile Navigationslösung erreicht.

Für eine praxisgerechte Anwendung in der Messung und Regelung von Fahrdynamikmanövern wird die Initialisierung der Sensorik und der Berechnung automatisiert, damit nach dem Einschalten des Gesamtsystems möglichst schnell und ohne Eingriff des Anwenders ein betriebsbereites Navigationssystem zur Verfügung zu steht.

Ein Zyklus der Berechnung des Kalman-Filters entspricht dem ungefähren Rechenzeitbedarf von 20 Strapdown-Zyklen. Bei einer Datenrate der IMU von 400Hz und einer Datenrate des DGPS-Empfängers von $20 \mathrm{~Hz}$ ergibt sich ein nahezu gleicher Rechenzeitbedarf für die beiden Hauptkomponenten der integrierten Navigationsrechnung. Die verfügbare Gesamtrechenleistung des Echtzeitrechners für das Bahnfolgeregelsystem (Abbildung 5.3) wird durch das integrierte Navigationssystem ungefähr zur Hälfte in Anspruch genommen.

\subsection{Berechnung fahrdynamikrelevanter Größen}

Wie bereits erwähnt liegen die Ausgangswerte des integrierten Navigationssystems zu jedem Abtastzeitpunkt $t_{n}$ zunächst für das Zentrum des inertialen Sensorpakets vor. Anhand der bekannten Orientierung $\boldsymbol{\rho}\left(t_{n}\right)$ und Winkelgeschwindigkeit $\boldsymbol{\omega}_{n b}^{b}\left(t_{n}\right)$ der Karosserie im Raum wird das Messergebnis von Position und Geschwindigkeit in den Fahrzeugschwerpunkt verschoben. Zusätzlich wird, wie auch schon festgestellt, die Messung von Position und Geschwindigkeit, welche in Navigationskoordinaten $n$ vorliegt, in das lokale ebene Koordinatensystem $t$ mit Ursprung im Mittelpunkt des Prüfgeländes transformiert. Von der so berechneten Position $\mathbf{p}\left(t_{n}\right)$ und Geschwindigkeit $\mathbf{v}\left(t_{n}\right)$ wird die vertikale Komponente nicht weiter berücksichtigt, da die Fahrversuche auf einer ebenen Fläche stattfinden. Aus der so erhaltenen Nord- und Ostkomponente der Geschwindigkeit im Schwerpunkt wird die Bewegungsrichtung $\theta\left(t_{n}\right)$ bestimmt. Die Differenz von Bewegungsrichtung $\theta\left(t_{n}\right)$ im Schwerpunkt und Gierwinkel $\psi\left(t_{n}\right)$ des Fahrzeugs ist der Schwimmwinkel $\beta\left(t_{n}\right)$ [4]. Mit $\mathbf{p}\left(t_{n}\right), \mathbf{v}\left(t_{n}\right), \theta\left(t_{n}\right)$ und $\psi\left(t_{n}\right)$ sind die für die Bahnfolgeregelung benötigten Messgrößen bestimmt.

Abbildung 3.11 zeigt die Position sowie das Geschwindigkeits-, Gierwinkel- und Schwimmwinkelprofil am Beispiel einer Runde auf dem Prüfgelände Weissach mit einem Fahrzeug vom Typ Porsche Carrera. Der Rundkurs wird im Uhrzeigersinn befahren. Die Messung beginnt an der Position (-270m, 90m) und endet nach einer Runde kurz 

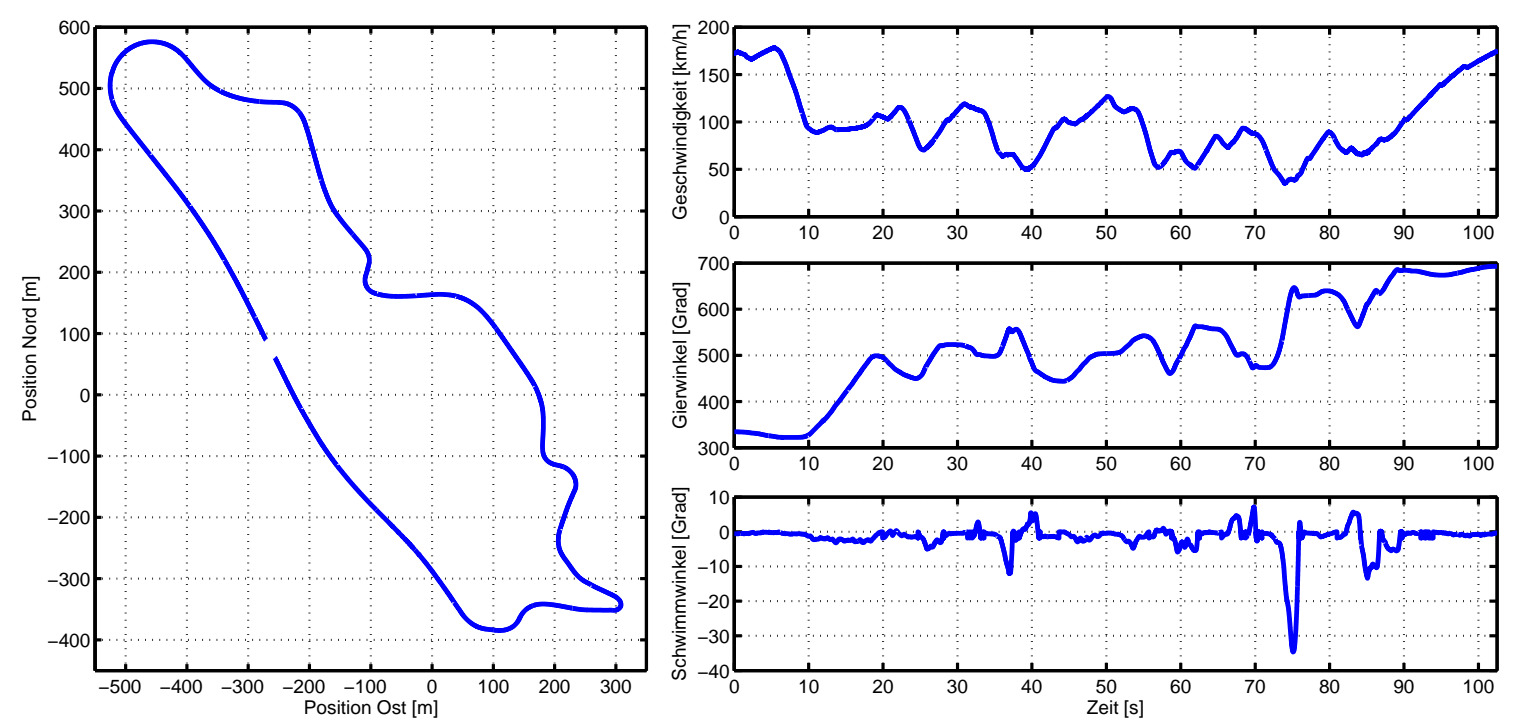

Abbildung 3.11: Ergebnisdaten des integrierten Navigationssystems für Position $\left(p_{N}(t), p_{E}(t)\right)$ in der Nord-Ost-Ebene, Absolutgeschwindigkeit $v(t)$, Gierwinkel $\psi(t)$ und Schwimmwinkel $\beta(t)$ bei einer Fahrt auf dem Prüfgelände in Weissach mit einem Fahrzeug vom Typ Porsche Carrera.

vor dieser Position. Die Messzeit beträgt ca. 102 Sekunden. Mit dem Verlauf der Geschwindigkeit und des Gierwinkels läßt sich der Zeitschrieb den jeweiligen Kurven des Streckenverlaufs zuordnen. Ein durch aggressive Fahrweise hervorgerufenes Ausbrechen des Fahrzeughecks ist an den stellenweise großen Schwimmwinkeln gut zu erkennen.

\subsection{Zusammenfassung}

Das in diesem Kapitel vorgestellte echtzeitfähige integrierte Navigationssystem liefert die messtechnische Voraussetzung, um den Regelkreis in Abbildung $1.2 \mathrm{zu}$ schließen. Durch die Verbindung eines inertialen Navigationssystems mit einem Satellitennavigationssystem besitzt die Messung des integrierten Navigationssystems die jeweils positiven Eigenschaften beider Systeme: Durch das Inertialsystem wird eine genaue Messung der Position und Orientierung mit ausreichend hoher Datenrate ermöglicht. Das Satellitennavigationssystem sorgt für die Langzeitstabilität des Navigationsergebnisses und eine reproduzierbare Positionserfassung.

Das beschriebene Navigationssystem stellt der Bahnfolgeregelung zu den Abtastzeitpunkten $t_{n}$ die Position $\mathbf{p}\left(t_{n}\right)$, die Geschwindigkeit $\mathbf{v}\left(t_{n}\right)$, die Bewegungsrichtung $\theta\left(t_{n}\right)$ und den Schwimmwinkel $\beta\left(t_{n}\right)$ der Karosserie im Fahrzeugschwerpunkt zur Verfügung. Diese Messgrößen werden sowohl für die Bahn- und Trajektorienplanung in Kapitel 2 als 
auch für die im folgenden Kapitel beschriebene Bahnfolgeregelung des Versuchsfahrzeugs benötigt.

Die Implementierung des integrierten Navigationssystem wurde auf fahrzeugtauglicher Hardware vorgenommen und in die Systematik der Porsche-Messtechnik eingebunden. So ist sowohl ein eigenständiger Betrieb des Navigationssystems als Komponente eines Messaufbaus als auch der Einsatz als Messsystem im Verbund mit der Querregelung möglich. Details der echtzeitfähigen Implementierung für die Anwendung in dem Gesamtsystem der Querregelung werden in Abschnitt 5.1.1 beschrieben. 


\section{Kapitel 4}

\section{Querregelung für die Bahnfolge eines Versuchsfahrzeugs}

Mit der in Kapitel 2 beschriebenen Trajektorienplanung sowie der Messung der Fahrzeugbewegung aus Kapitel 3 sind die Voraussetzungen gegeben, damit der Regelkreis für die Bahnfolgeregelung entsprechend Abbildung 1.2 geschlossen werden kann. Die Aufgabe der Folgeregelung ist es, den Querabstand des Fahrzeugschwerpunktes zu der Sollbahn zu minimieren. Der Querabstand stellt die Regelgröße der Folgeregelung dar. Die dazu verfügbare Stellgröße ist der Lenkradwinkel.

Die Folgeregelung soll mit der in Abbildung 1.2 gezeigten Struktur mit einer Vorsteuerung und einem Regler realisiert werden. Diese Struktur erlaubt es, das Führungs- und Störverhalten unabhängig voneinander zu entwerfen [38]. Die Steuergröße ist durch die Sollbahnkrümmung aus der Trajektorienplanung gegeben.

Für den modellbasierten Entwurf der Vorsteuerung wird ein inverses Fahrzeugmodell benötigt. Das zugrundeliegende Fahrzeugmodell soll sowohl bei Stillstand und langsamer Fahrt als auch bei maximaler Fahrzeugquerbeschleunigung bestmöglich mit dem realen Fahrzeug übereinstimmen, um einen hohen Vorsteueranteil der Bahnfolgeregelung zu erreichen. Um Modellunsicherheiten und Störgrößen auszugleichen, wird der Querabstand des Fahrzeugschwerpunktes zu der Sollbahn zusätzlich zu der Vorsteuerung durch eine lineare Regelung minimiert.

Nach einer simulationstechnischen Überprüfung der entworfenen Querregelung wird diese auf einer echtzeitfähigen und fahrzeugtauglichen Rechnerplattform implementiert.

\subsection{Fahrzeugmodellierung}

Für den modellbasierten Entwurf der Querregelung wird ein geeignetes Fahrzeugmodell benötigt. Um einen praxistauglichen Einsatz der Bahnfolgeregelung sowohl bei langsamer 
Querregelung für die Bahnfolge eines Versuchsfahrzeugs

Fahrt als auch im querdynamischen Grenzbereich zu ermöglichen, werden für den Entwurf der Querregelung insgesamt drei unterschiedliche Fahrzeugmodelle verwendet.

Bei langsamer Fahrt mit Geschwindigkeiten kleiner als $20 \mathrm{~km} / \mathrm{h}$ wird der für die gewünschte Bahnkrümmung notwendige Lenkradwinkel mithilfe eines kinematischen Modells (Abschnitt 4.1.1) bestimmt. Dieses erste Modell basiert auf der Annahme, daß die Räder des Fahrzeugs ohne Schlupf auf der Fahrbahn abrollen.

Für („hohe“) Geschwindigkeiten größer als 30km/h basiert der Vorsteuerungsentwurf auf einem dynamischen Fahrzeugmodell (Abschnitt 4.1.2). In diesem zweiten Modell wird im Gegensatz zu dem kinematischen Modell berücksichtigt, daß das Abrollen der Reifen schlupfbehaftet ist. Aus dem Reifenschlupf resultieren Reifenseitenkräfte, die der Querbeschleunigung des Fahrzeugs bei Kurvenfahrt entgegenwirken. Einerseits berücksichtigt das dynamische Fahrzeugmodell die nichtlinearen Reifeneigenschaften, um eine ausreichende Modellgüte für den fahrdynamischen Grenzbereich zu erzielen, andererseits ist es einfach genug, um als Grundlage für einen modellbasierten Vorsteuerungsentwurf zu dienen.

Für den Übergangsbereich mit Geschwindigkeiten zwischen 20 und 30km/h basiert die Folgeregelung entsprechend gewichtet sowohl auf dem kinematischen als auch auf dem dynamischen Modell, um einen sanften Wechsel zu erreichen.

Das dritte in dieser Arbeit verwendete Modell ist ein Fahrzeugmodell auf Basis der Simulationsumgebung von MatLab/Simulink. Dieses Modell existiert sowohl für die Baureihe des Porsche Carrera als auch für die Baureihe des Porsche Cayenne. Es wurde anhand von Messungen an realen Fahrzeugen validiert und bildet das Fahrzeugverhalten detailliert ab. Dieses Modell wird in in der Porsche AG unter anderem für die Weiterentwicklung der Fahrzeuge verwendet. Es dient in der vorliegenden Arbeit neben der simulativen Überprüfung der entworfenen Querregelung auch der Ermittlung der Reifenkennlinien für das dynamische Fahrzeugmodell unter Vermeidung aufwendiger Versuche und Messaufbauten.

In diesem Abschnitt werden sowohl das für den Entwurf der Folgeregelung verwendete kinematische und dynamische Fahrzeugmodell als auch die Gewinnung der Reifenkennlinien mithilfe des detaillierten Modells beschrieben.

\subsubsection{Kinematisches Fahrzeugmodell}

Das kinematische Einspurmodell ist ein stark vereinfachtes Modell eines PKW (Abbildung 4.1). Die vorderen und hinteren Räder sind zu jeweils einem Rad vorne und hinten zusammengefaßt. Durch die Annahme des perfekten Abrollens der Räder auf der Fahrbahn fallen die Kurvenmittelpunkte aller Räder und Ersatzräder in einem einzigen Punkt C zusammen. Die Freiheitsgrade des Modells beschränken sich auf die Bewegung 


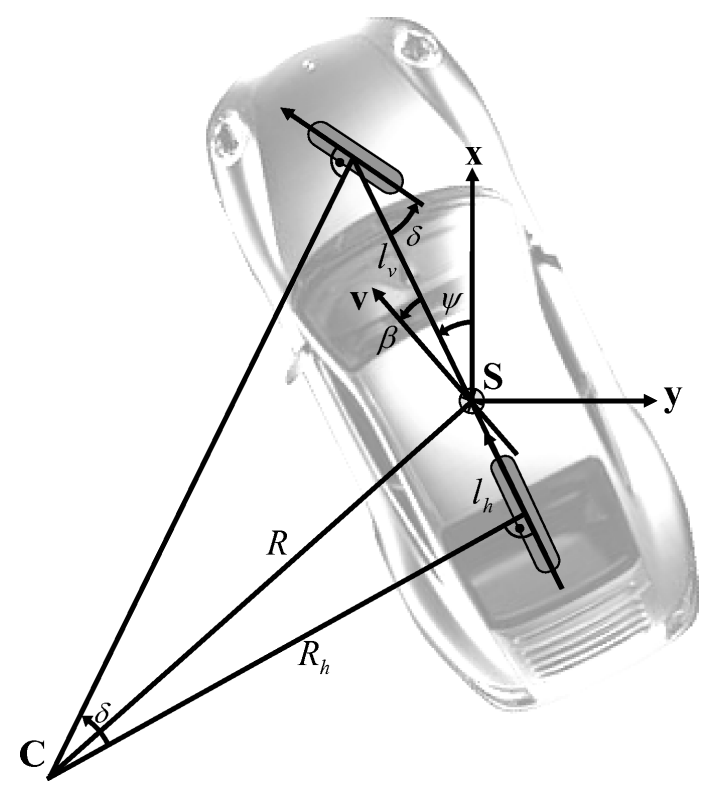

\author{
S: Fahrzeugschwerpunkt \\ C: Kurvenzentrum \\ $(\mathbf{x}, \mathbf{y})$ : Fahrbahnebene \\ $l_{v}, l_{h}$ : Entfernung Schwerpunkt-Rad \\ $\delta$ : Lenkwinkel \\ $R$ : Bahnradius am Schwerpunkt \\ $R_{h}$ : Bahnradius am Hinterrad \\ $\mathbf{v}$ : Geschwindigkeitsvektor in $\mathbf{S}$ \\ $\psi$ : Gierwinkel \\ $\beta$ : Schwimmwinkel
}

Abbildung 4.1: Kinematisches Fahrzeugmodell (4.2) für langsame Fahrt ohne Reifenschlupf. Positive Winkel sind in Übereinstimmung mit [4] gegen den Uhrzeigersinn gerichtet.

des Schwerpunktes $\mathbf{S}(x, y)$ in der $(x, y)$-Ebene und die Drehung $\psi$ des Fahrzeugs um die Hochachse. Aus der Kinematik ergeben sich der Bahnradius am Hinterrad

$$
R_{h}=\frac{\left(l_{v}+l_{h}\right)}{\tan \delta}
$$

und der Bahnradius am Fahrzeugschwerpunkt

$$
R=\frac{1}{\tan \delta} \sqrt{\left(l_{v}+l_{h}\right)^{2}+l_{h}^{2}(\tan \delta)^{2}}
$$

aus dem Lenkwinkel $\delta$ und den Abständen $l_{v}$ und $l_{h}$ des Vorder- und Hinterrades zu dem Fahrzeugschwerpunkt. Ein positiver Wert von $R$ steht für eine Linkskurve, ein negativer Wert von $R$ steht für eine Rechtskurve. Da ein Geradenstück mit Lenkwinkel $\delta \rightarrow 0$ in (4.2) einen unendlichen Radius hat und somit numerisch nicht darstellbar ist, ist der Bahnradius $R$ nur eingeschränkt für die Beschreibung der Sollbahn geeignet. Als Alternative zu dem Bahnradius wird die Bahnkrümmung $\kappa$ daher als Kehrwert des Radius $R$ definiert:

$$
\kappa=\frac{1}{R} \quad \text { für } R \neq 0 \text {. }
$$

Analog zu dem Bahnradius liegt für positive Werte von $\kappa$ eine Linkskurve vor, für negative Werte von $\kappa$ eine Rechtskurve. Die Bahnkrümmung ist durch den maximalen Lenkwinkel begrenzt. In der Bahn des Fahrzeugschwerpunktes können daher keine Knicke (unendliche Krümmung, Radius null) auftreten. 


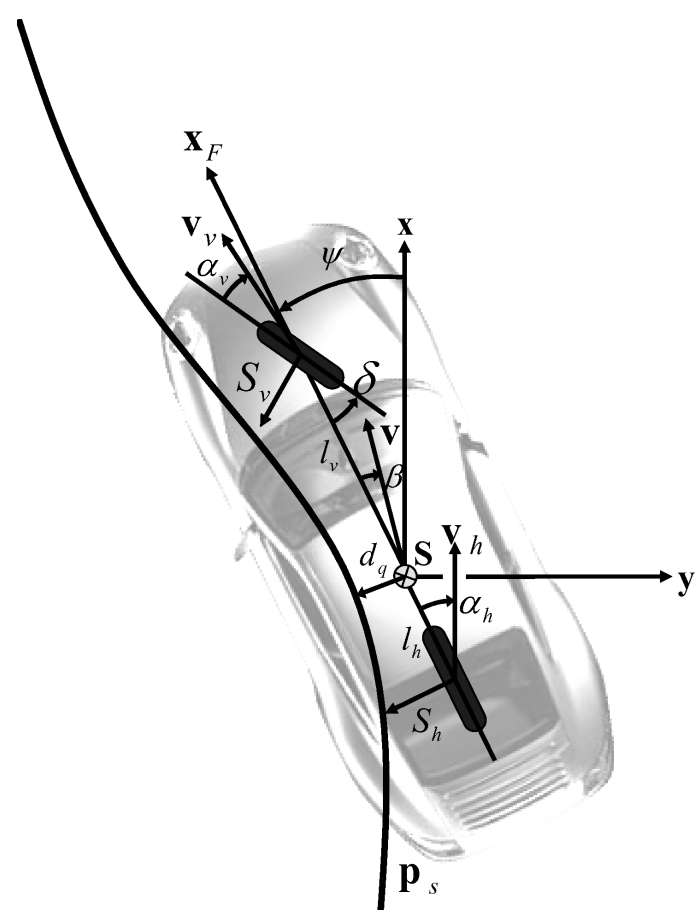

\author{
S: Fahrzeugschwerpunkt \\ $(\mathbf{x}, \mathbf{y})$ : Fahrbahnebene \\ $\mathbf{x}_{F}$ : Fahrzeuglängsachse \\ $l_{v}, l_{h}:$ Entfernung Schwerpunkt-Rad \\ $\delta$ : Lenkwinkel \\ $\alpha_{v}, \alpha_{h}: \quad$ Schräglaufwinkel \\ $S_{v}, S_{h}$ : Reifenseitenkraft \\ $\mathbf{v}_{v}, \mathbf{v}_{h}$ : Radgeschwindigkeiten \\ v: Geschwindigkeitsvektor in $S$ \\ $\psi$ : Gierwinkel \\ $\beta$ : Schwimmwinkel \\ $d_{q}:$ Abstand zur Sollbahn \\ $\mathbf{p}_{s}:$ Sollbahn
}

Abbildung 4.2: Systemgrößen und Parameter für das dynamische Einspurmodell (4.4)(4.7) nach Riekert und Schunck [28]. Positive Winkel sind in Übereinstimmung mit [4] gegen den Uhrzeigersinn gerichtet.

Die Fahrzeuggeschwindigkeit $\mathbf{v}$ kommt in Gleichung (4.2) nicht vor. Der Kurvenradius und die Bahnkrümmung nach (4.2)-(4.3) sind daher bei kinematischer Fahrt von der Geschwindigkeit unabhängig und auch bei Fahrzeugstillstand oder Rückwärtsfahrt gültig. Da die Annahmen für das kinematische Modell nur für langsame, querbeschleunigungsarme Fahrt bei vernachlässigbarem Reifenschlupf realistisch sind, muß bei höheren Geschwindigkeiten ein dynamisches Fahrzeugmodell verwendet werden.

\title{
4.1.2 Dynamisches Fahrzeugmodell
}

Das Entwurfsmodell für die schnelle Fahrt, welches in Abbildung 4.2 skizziert ist, basiert auf dem Einspurmodell von Riekert und Schunck [28]. Der Fahrzeugschwerpunkt $S$ in der $(x, y)$-Ebene hat den Abstand $l_{v}$ und $l_{h}$ zu dem vorderen und hinteren Radmittelpunkt. Der Geschwindigkeitsvektor $\mathbf{v}$ des Schwerpunktes schließt mit der Fahrzeuglängsachse $\mathbf{x}_{F}$ den Schwimmwinkel $\beta$ ein. Die Orientierung der Fahrzeuglängsachse bezüglich der Nordrichtung (identisch mit der $x$-Achse) wird als der bereits eingeführte Gierwinkel $\psi$ bezeichnet. Der Winkel zwischen der Fahrzeuglängsachse und der Orientierung des Vorderrades ist der Lenkwinkel $\delta$. Der Unterschied zu dem kinematischen Modell in Abbildung 4.1 besteht darin, daß ein schlupfbehaftetes Abrollen der Räder zugelassen wird. 
Daher sind die Geschwindigkeitsvektoren $\mathbf{v}_{v}$ und $\mathbf{v}_{h}$ im Mittelpunkt der Räder nicht mit deren Orientierung identisch. Die beiden Schräglaufwinkel $\alpha_{v}$ ud $\alpha_{h}$ bezeichnen diese Winkeldifferenz. Aufgrund der Schräglaufwinkel entstehen die beiden Reifenseitenkräfte $S_{v}$ vorne und $S_{h}$ hinten, welche orthogonal zu der Reifenorientierung wirken. Der Abstand $d_{q}$ wurde bereits in Kapitel 2 als Regelgröße definiert und bezeichnet den Abstand des Fahrzeugschwerpunktes von der Sollbahn. Die Modellgleichungen von Riekert und Schunck [28]

$$
\begin{aligned}
m v(\dot{\psi}+\dot{\beta}) & =S_{v}\left(\alpha_{v}\right) \cos (\delta-\beta)+S_{h}\left(\alpha_{h}\right) \cos (\beta) \\
J \ddot{\psi} & =S_{v}\left(\alpha_{v}\right) l_{v} \cos (\delta)-S_{h}\left(\alpha_{h}\right) l_{h}
\end{aligned}
$$

mit

$$
\begin{aligned}
& \alpha_{v}=\delta-\arctan \left(\frac{l_{v} \dot{\psi}+v \sin \beta}{v \cos \beta}\right), \\
& \alpha_{h}=\arctan \left(\frac{l_{h} \dot{\psi}-v \sin \beta}{v \cos \beta}\right)
\end{aligned}
$$

umfassen die Kräftebilanz (4.4) in der Ebene, projiziert in Richtung der Bahnquerbeschleunigung sowie die Momentenbilanz (4.5) um die Hochachse. Die Reifenseitenkräfte $S_{v}$ und $S_{h}$ darin sind abhängig von den Reifenschräglaufwinkeln $\alpha_{v}$ und $\alpha_{h}$ nach (4.6) und (4.7). Der hintere Schräglaufwinkel $\alpha_{h}$ (4.6) ist von dem Schwimmwinkel $\beta$, der Gierrate $\dot{\psi}$ und der Fahrzeuggeschwindigkeit v abhängig. Der vordere Schräglaufwinkel $\alpha_{v}$ kann zusätzlich durch den Lenkwinkel $\delta$ verändert werden. Die Wahl der Kennlinien $S_{v}\left(\alpha_{v}\right)$ und $S_{h}\left(\alpha_{h}\right)$, die die Abhängigkeit der Reifenseitenkraft von dem Schräglaufwinkel beschreiben, ist entscheidend für die Genauigkeit des dynamischen Einspurmodells. Für stationäre Kurvenfahrt kann die für das Einspurmodell angenommene Veinfachung, dass der Fahrzeugschwerpunkt in der Fahrbahnebene liegt, durch geeignete Wahl dieser Kennlinien teilweise kompensiert werden (siehe Abschnitt 4.1.3).

Für die Kräftebilanz (4.4) muß die Zentrifugalkraft $m v(\dot{\psi}+\dot{\beta})$ durch die Reifenseitenkräfte $S_{v}\left(\alpha_{v}\right)$ vorne und $S_{h}\left(\alpha_{h}\right)$ hinten ausgeglichen werden. Dabei wird nur die zu der Richtung der Fahrzeuggeschwindigkeit $\mathbf{v}$ orthogonale Komponente der Reifenseitenkräfte herangezogen. Die Kraftkomponenten in Bewegungsrichtung bleiben in der Kräftebilanz unberücksichtigt, da sie lediglich die Fahrzeuggeschwindigkeit betreffen und durch die einzige Stellgröße, den Lenkradwinkel, nur mittelbar beeinflußt werden. Die Momentenbilanz (4.5) enthält das Massenträgheitsmoment $J$ des Fahrzeugs um die Hochachse, die Gierwinkelbeschleunigung $\ddot{\psi}$ des Fahrzeugs und die Reifenseitenkräfte $S_{v}$ und $S_{h}$, welche über die Hebelarme der Länge $l_{v}$ und $l_{h}$ wirken.

Die Reifenkräfte $S_{v}\left(\alpha_{v}\right)$ und $S_{h}\left(\alpha_{h}\right)$ entstehen durch eine von der Längsachse des Reifens unterschiedliche Reifenbewegungsrichtung, den erwähnten Schräglauf des Reifens auf der 


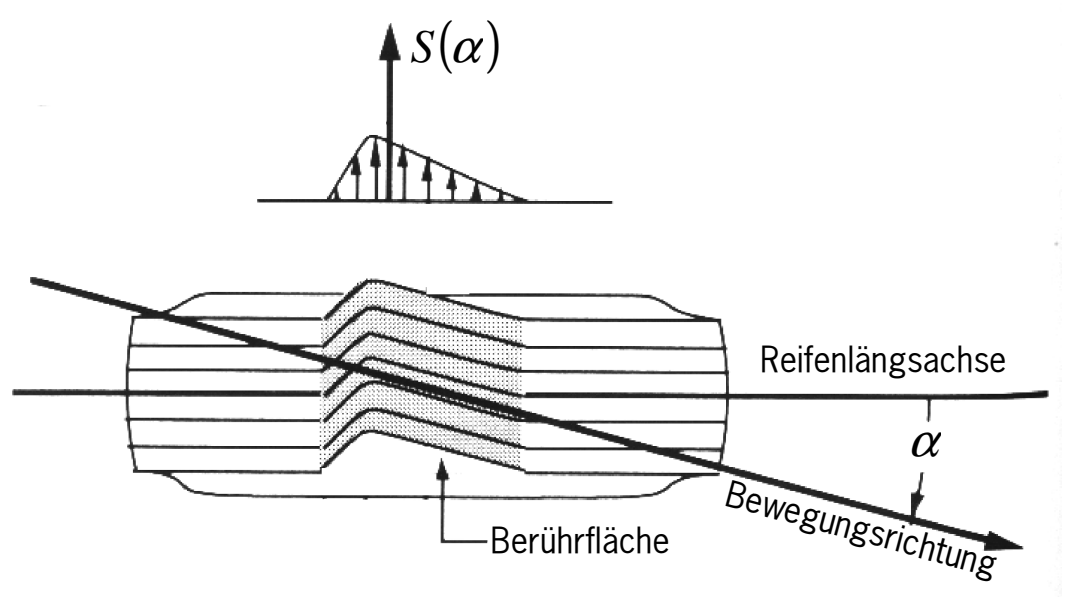

Abbildung 4.3: Entstehung der Seitenkraft $S(\alpha)$ durch schlupfbehaftetes Abrollen des Reifens auf der Fahrbahn mit dem Schräglaufwinkel $\alpha$ (Abbildung aus [22]).

Fahrbahnoberfläche (Abbildung 4.3). Durch den Reifenschräglaufwinkel $\alpha$ werden die Profilblöcke des Reifens im Bereich der Berührfläche von Reifen und Fahrbahn wie in Abbildung 4.3 überhöht skizziert ausgelenkt, bis die rückstellende Kraft beim Abrollen die Haftreibung überschreitet und die Profilblöcke wieder in Ihre Ausgangslage gleiten. Die Summe aller so entstehenden Feder- und Gleitreibungskräfte ergibt die Reifenseitenkraft $S(\alpha)$ [22]. Die Reifenkräfte $S_{v}\left(\alpha_{v}\right)$ und $S_{h}\left(\alpha_{h}\right)$ in (4.4) und (4.5) lassen sich mithilfe der Reifenkennlinien in Abbildung 4.4 über die Schräglaufwinkel $\alpha_{v}$ und $\alpha_{h}$ vorne und hinten ermitteln.

Um die Bewegung des Fahrzeugschwerpunktes in der Ebene durch die Modellgleichungen (4.4)-(4.7) zu beschreiben, wird mit

$$
(\dot{\psi}+\dot{\beta})=\kappa v
$$

die Änderung der Bewegungsrichtung $(\dot{\psi}+\dot{\beta})$ in $(4.4)$ über die Bahnkrümmung $\kappa$ des Fahrzeugschwerpunktes und dessen Geschwindigkeit $v$ ausgedrückt. Durch die Bahnkrümmung $\kappa$ in der Modellgleichung (4.4) wird die Verbindung zu der Sollbahnplanung in Kapitel 2.3 geschaffen, welche die Sollbahnkrümmung $\kappa_{s}$ (2.13) des Fahrzeugschwerpunktes für die modellbasierte Bahnfolgeregelung zur Verfügung stellt.

Die für das dynamische Fahrzeugmodell (4.4)-(4.7) benötigten Modellparameter sind die Fahrzeugmasse $m$, die Entfernung $l_{v}$ und $l_{h}$ der Vorder- und Hinterachse vom Schwerpunkt, das Massenträgheitsmoment $J$ und die Reifenkennlinien $S_{v}\left(\alpha_{v}\right)$ und $S_{h}\left(\alpha_{h}\right)$. Da der Ermittlung der Reifenkennlinien für die Realitätstreue des Fahrzeugmodells eine besondere Bedeutung zukommt, wird die Ermittlung der Reifenkennlinien im folgenden Unterkapitel gesondert beschrieben. 


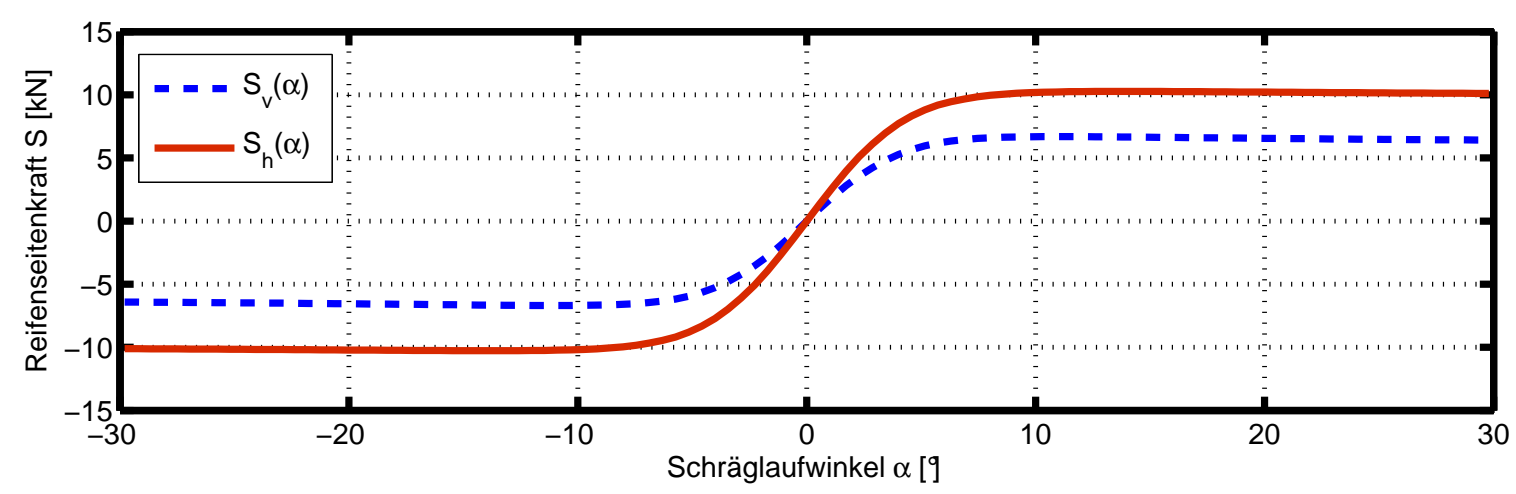

Abbildung 4.4: Aus der Simulation des detaillierten Fahrzeugmodells eines Porsche Carrera gewonnene Reifenkennlinien $S_{v}(\alpha)$ und $S_{h}(\alpha)$

\subsubsection{Reifenkennlinien für stationäre Fahrt}

Die Reifenseitenkraft $S$ ist in nichtlinearer Weise von dem Reifenschräglaufwinkel $\alpha$ abhängig. Sie hängt daneben von vielen weiteren Parametern und Variablen wie z.B. dem Reifentyp, dem Reifenverschleiß, der Fahrbahnbeschaffenheit, der Reifenaufstandskraft und den zusätzlich zu übertragenden Längskräften ab. Der Reifentyp und die Fahrbahnbeschaffenheit sind bei Fahrdynamikversuchen bekannt beziehungsweise konstant. Den nächstgrößten Einfluß auf die Reifenseitenkraft hat die vertikale Reifenaufstandskraft. Das Einspurmodell bildet die Änderung der Reifenaufstandskraft bei Kurvenfahrt zunächst nicht ab. Um diese Vereinfachung des Modells zumindest teilweise zu kompensieren, wird die durch den hochliegenden Schwerpunkt bedingte Lastumverteilung von den kurveninneren zu den kurvenäußeren Rädern bei der Ermittlung der Reifenkennlinien für das dynamische Fahrzeugmodell berücksichtigt. Eine erhöhte Reifenaufstandkraft bedeutet eine höhere maximale Reifenseitenkraft. Allerdings steigt die maximale Reifenseitenkraft unterproportional mit der Reifenaufstandskraft.

Zur Identifikation der Reifenkennlinien unter Berücksichtigung der hochliegenden Schwerpunktlage wird das detaillierte Fahrzeugmodell des betreffenden Fahrzeugs simuliert. Das validierte Fahrzeugmodell berücksichtigt die veränderlichen Aufstandskräfte der vier Reifen. Die simulierte Fahrt wird bei verschiedenen Geschwindigkeiten und Lenkwinkeln durchgeführt. Für den sich einstellenden stationären Zustand des simulierten Fahrzeugs ermöglicht die Simulationsumgebung das Auslesen der Reifenseitenkräfte der vier Reifen bei den auftretenden Reifenschräglaufwinkeln. Diese Werte dienen als Stützpunkte für die gesuchten Reifenkennlinien der Vorder- und Hinterräder. Hierbei ist darauf zu achten, daß ein Bereich der Schräglaufwinkel an den Vorder- und Hinterrädern bis über die Seitenkraft-Maxima abgedeckt wird, um später die Reifenkennlinien auch für die großen Reifenschräglaufwinkel eines ausbrechenden Fahrzeugs zur Verfügung zu haben. Wird nun die Summe der jeweils vorderen und hinteren Reifenseitenkräfte gebildet, läßt sich 
eine Reifenkennlinie für das Einspurmodell gewinnen, die den tatsächlichen Reifenseitenkräften bei Verfolgung der geplanten glatt verlaufenden Sollbahn, also bei quasistationärer Vertikallast der einzelnen Reifen, sehr ähnlich ist (Abbildung 4.4). So werden die bei stationärer Kurvenfahrt auftretenden Radlaständerungen durch die Reifenkennlinien $S_{v}\left(\alpha_{v}\right)$ und $S_{h}\left(\alpha_{h}\right)$ indirekt in dem Einspurmodell (4.4)-(4.7) berücksichtigt. Die hintere Reifenseitenkraft $S_{h}$ lässt sich durch die Messung des Reifenschräglaufwinkels $\alpha_{h}$ über die Reifenkennlinie bestimmen. Die vordere Reifenseitenkraft $S_{v}$ wird für den modellbasierten Reglerentwurf als Systemeingang betrachtet, da der vordere Reifenschräglaufwinkel $\alpha_{v}$ mit Kenntnis des messbaren Fahrzeugzustands und der Kennlinie der Lenkkinematik über die Lenkmaschine gesteuert werden kann.

Sollte für künftig zu regelnde Fahrzeuge (Neuentwicklungen oder Fremdfabrikate) noch kein detailliertes Fahrzeugmodell zur Verfügung stehen, können die Reifenkennlinien anstatt mit dem detaillierten Simulationsmodell auch mit Hilfe von Messrädern im realen Versuch identifiziert werden. Dann werden die benötigten Größen bei verschiedenen Geschwindigkeiten und Lenkwinkeln nicht simulativ, sondern messtechnisch am realen Fahrzeug unter den relevanten Versuchsbedingungen erfaßt.

Die Reifenkennlinien sind für die spätere Implementierung in Form von Stützpunkten abzuspeichern, um in der modellbasierten Bahnfolgeregelung mit möglichst geringem Rechenaufwand durch Interpolation rekonstruiert werden zu können. Ist die Sollbahn wie in Kapitel 2 beschrieben glatt und ruckarm, findet eine quasistationäre Kurvenfahrt des Fahrzeugs statt. Unter dieser Voraussetzung und mit Reifenkennlinien, die entsprechend der obigen Beschreibung identifiziert werden, bildet das verhältnismäßig einfache Einspurmodell (4.4)-(4.7) das reale Fahrzeug für den Reglerentwurf im fahrdynamischen Grenzbereich (Abschnitt 4.2) hinreichend genau ab.

\subsection{Regelungsstruktur mit zwei Freiheitsgraden}

Die Aufgabe der Folgeregelung ist es, den Querabstand $d_{q}$ des Fahrzeugschwerpunktes $S$ zur Sollbahn $\mathbf{p}_{s}$ zu minimieren (Abbildung 4.2). Dazu wird für die Bahnfolgeregelung eine Regelungsstruktur mit zwei Freiheitsgraden verwendet (Abbildung 4.5). Die zu entwerfende Vorsteuerung und Regelung bauen auf der Trajektorienplanung (Kapitel 2) und dem Navigationssystem (Kapitel 3) auf. Als Ergebnis muß der Lenkmaschine zu jedem Abtastzeitpunt der Soll-Lenkradwinkel $\delta_{L 1}$ zur Verfügung gestellt werden. Durch die Unterscheidung von kinematischem und dynamischem Modell in Abschnitt 4.1 unterscheiden sich auch die dazugehörige Vorsteuerung und Regelung. In Abschnitt 4.2.1 werden zunächst die Vorsteuerung und Regelung für langsame Fahrt beschrieben. Darauf aufbauend folgt in Abschnitt 4.2.2 der Entwurf der Vorsteuerung und Regelung für schnelle Fahrt. In Abschnitt 4.2.3 werden die beiden Folgeregler zu einer Folgeregelung für den gesamten Geschwindigkeitsbereich zusammengefügt. 


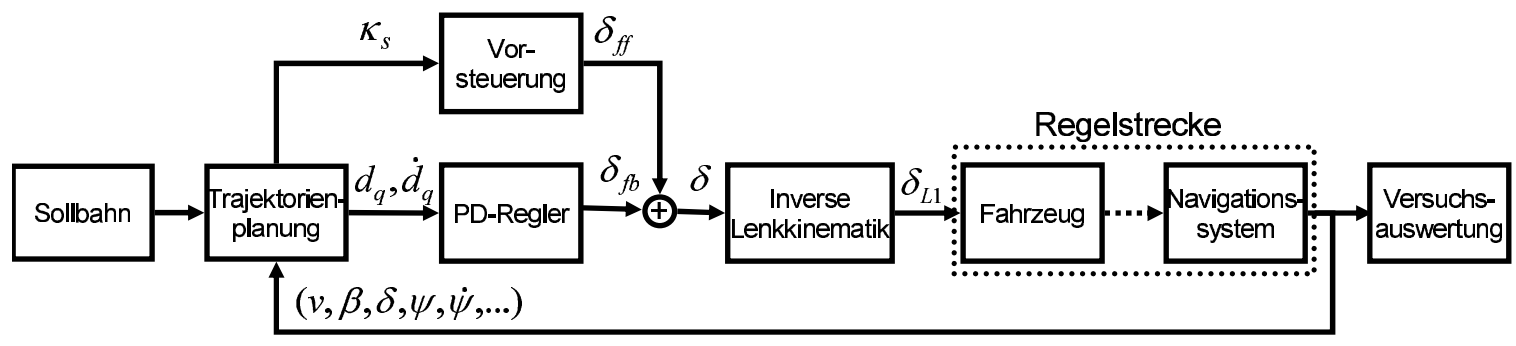

Abbildung 4.5: Blockschaltbild für die Querregelung eines Fahrzeugs auf der Basis des kinematischen Fahrzeugmodells (4.2)

\subsubsection{Folgeregelung für langsame Fahrt}

Bei Stillstand oder bei sehr geringen Geschwindigkeiten (z.B. kleiner 20km/h) läßt sich der für die Reifenkraftbestimmung benötigte Schwimmwinkel $\beta$ nicht oder nur sehr ungenau bestimmen. Bei diesen geringen Geschwindigkeiten sind die Querbeschleunigungen entsprechend gering und damit die Annahme einer Fahrt ohne Reifenschlupf realistisch.

Abbildung 4.5 zeigt die Struktur der Querregelung für den kinematischen Fall. Die Trajektorienplanung liefert die Sollbahnkrümmung $\kappa_{s}$ für die Vorsteuerung und die Regelabweichungen $d_{q}$ und $\dot{d}_{q}$ für den Regler. Die Summe der Lenkwinkel aus Vorsteuerung $\delta_{f f}$ und Regelung $\delta_{f b}$ ergibt den für die Bahnfolge benötigten Lenkwinkel $\delta$. Über den in Abbildung 4.5 dargestellten Block mit der inversen Lenkkinematik wird aus $\delta$ der SollLenkradwinkel $\delta_{L 1}$ berechnet und mithilfe der Lenkmaschine an das Fahrzeug übergeben.

\section{Inversionsbasierte Vorsteuerung für langsame Fahrt}

Für die Vorsteuerung muß das kinematische Modell (4.2) invertiert werden, um aus der vorgegebenen Solltrajektorie zu jedem Zeitpunkt $t$ den benötigten Lenkwinkel $\delta_{f f}(t)$ zu erhalten. Die Solltrajektorie wird wie in Abbildung 4.5 dargestellt durch die Sollbahnkrümmung $\kappa_{s}\left(t^{\star}\right)$ im Fahrzeugschwerpunkt vorgegeben. So ergibt sich aus den Gleichungen (4.2) und (4.3) der für eine vorgegebene Sollbahnkrümmung $\kappa_{s}$ benötigte Lenkwinkel

$$
\delta_{f f}(t)=\arctan \left[\left(l_{h}+l_{v}\right) \kappa_{s}\left(t^{\star}\right)\left(1-l_{h}^{2} \kappa_{s}^{2}\left(t^{\star}\right)\right)^{-1 / 2}\right] \text { für } \kappa_{s}\left(t^{\star}\right)<1 / l_{h} .
$$

Die Berechnung ist von der Fahrzeuggeschwindigkeit unabhängig und daher auch für das Anfahren und Anhalten bis zum Fahrzeugstillstand anwendbar. Als leicht zu bestimmende Modellparameter werden für (4.9) die Schwerpunktlage $l_{h}$ und der Achsabstand $l_{v}+l_{h}$ des Fahrzeugs benötigt.

\section{PD-Regler}

Modellfehler und Störeinflüsse werden durch den Regelanteil des Lenkwinkels

$$
\delta_{f b}(t)=p_{k 1} d_{q}(t)+p_{k 2} \dot{d}_{q}(t)
$$


mit dem Querabstand $d_{q}$ in (2.14) und der Annäherungsgeschwindigkeit $\dot{d}_{q}$ in (2.15) kompensiert. Die Parameter $p_{k 1}$ und $p_{k 2}$ in (4.10) werden mit Hilfe von Simulation heuristisch bestimmt und später im Experiment am realen Fahrzeug feinjustiert. Durch die einfache Struktur des PD-Reglers (4.10) für langsame Fahrt läßt sich dessen Parametrierung auch während einer Erprobung im Bedarfsfall vom Versuchsingenieur problemlos ändern.

\section{Inverse Lenkkinematik}

Als Summe des Vorsteuerungs- und Regelanteils entsteht der für die Bahnfolge benötigte Lenkwinkel

$$
\delta(t)=\delta_{f f}(t)+\delta_{f b}(t) .
$$

Der in Abbildung 4.5 dargestellte Block der inversen Lenkkinematik hat die Aufgabe, ausgehend von dem benötigten vorderen Lenkwinkel $\delta$ den dazugehörigen Lenkradwinkel $\delta_{L 1}$ als Steuergröße zu bestimmen. Unter der Annahme einer steifen, hysteresefreien Lenkung und damit eines eindeutigen Zusammenhangs zwischen dem Lenkwinkel $\delta$ und dem Lenkradwinkel $\delta_{L 1}$, wird $\delta_{L 1}$ über die Kennlinie ermittelt, welche die Lenkkinematik des Fahrzeugs repräsentiert. Für kleine Lenkwinkel und konstante Lenkuntersetzung ist diese Kennlinie eine Ursprungsgerade mit einer Steigung entsprechend der Lenkuntersetzung.

\section{Zusammenfassung}

Die vorgestellte Vorsteuerung und Regelung auf Basis des kinematischen Fahrzeugmodells ermöglicht eine Querregelung für kleine Geschwindigkeiten bis zum Fahrzeugstillstand. Die Verwendung des kinematischen Modells für die Vorsteuerung ist in diesem Fall ausreichend und ergibt einen ruhigen Verlauf des Lenkradwinkels, da dieser von nur wenigen und rauscharm zu erfassenden Messgrößen abhängt. Für die Querregelung bei höheren Querbeschleunigungen des Fahrzeugs wird die im folgenden Abschnitt beschriebene Folgeregelung auf der Basis des dynamischen Fahrzeugmodells verwendet.

\subsubsection{Folgeregelung für schnelle Fahrt}

Für die Folgeregelung bei schneller Fahrt unter Berücksichtigung von Reifenschlupf soll wie bei der Folgeregelung für langsame Fahrt aus Abschnitt 4.2.1 ausgehend von der Sollbahnkrümmung $\kappa_{s}$ und den Regelabweichungen $d_{q}$ und $\dot{d}_{q}$ der für die Bahnfolge benötigte Lenkradwinkel $\delta_{L 2}$ ermittelt werden.

In dem dynamischen Fahrzeugmodell (4.5)-(4.7) stellen die Reifenseitenkräfte die Verbindung zwischen Fahrbahn und Fahrzeug her. Eine Änderung des Lenkwinkels $\delta$ hat direkt eine Änderung der vorderen Reifenseitenkraft zur Folge. In Abbildung 4.6 ergibt 


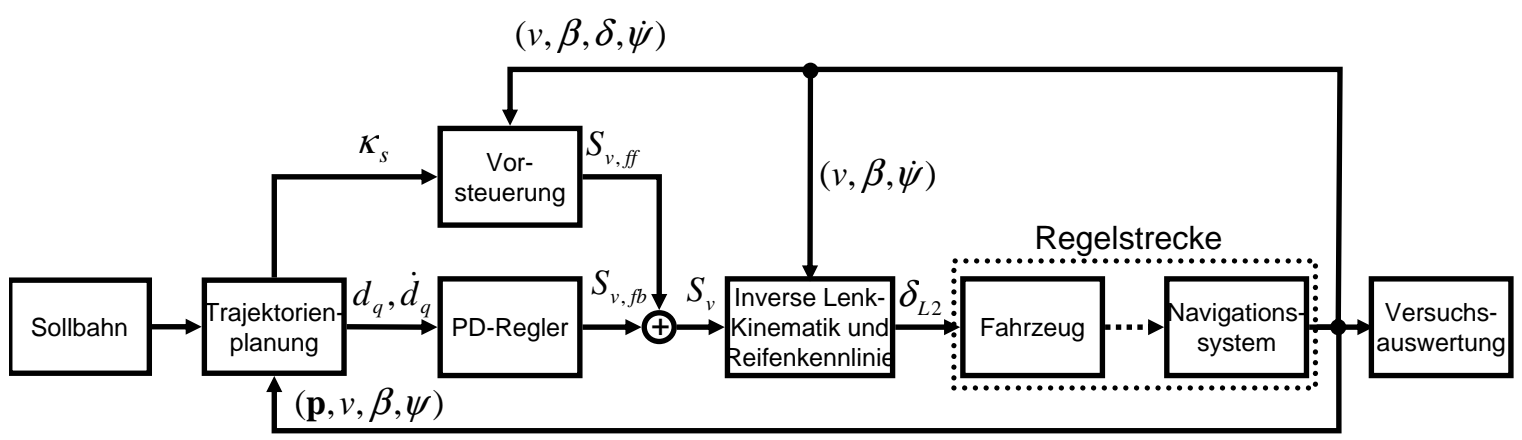

Abbildung 4.6: Blockschaltbild für die modellbasierte Querregelung eines Fahrzeugs auf Basis des dynamischen Fahrzeugmodells (4.4)-(4.7)

die Summe aus Vorsteuerung $S_{v, f f}$ und Regelung $S_{v, f b}$ die benötigte vordere Reifenseitenkraft $S_{v}$ und nicht wie bei der kinematischen Folgeregelung in Abbildung 4.5 direkt den benötigten Lenkwinkel $\delta$. Aus $S_{v}$ wird in dem nachgeschalteten Block mithilfe der inversen Fahrzeugkinematik, der Reifeneigenschaften und dem gemessenen Fahrzeugzustand der für die Bahnfolge benötigte Lenkradwinkel $\delta_{L 2}$ berechnet.

\section{Inversionsbasierte Vorsteuerung für schnelle Fahrt}

Für den Entwurf der inversionsbasierten Vorsteuerung wird die Kräftebilanz (4.4) nach der vorderen Reifenseitenkraft

$$
S_{v}(t)=\frac{m v(\dot{\psi}+\dot{\beta})-S_{h}\left(\alpha_{h}\right) \cos \beta}{\cos (\delta-\beta)}
$$

aufgelöst. Dabei wird die Momentenbilanz (4.5) nicht berücksichtigt, da die Kräfte zum Aufbringen des nötigen Giermomentes bei Einleitung einer Kurvenfahrt für Personenkraftwagen klein sind gegenüber den nötigen Reifenseitenkräften zur Kompensation der Zentrifugalkraft.

Das Fahrzeug soll sich auf der Sollbahn bewegen. Daher wird für die Änderung der Bewegungsrichtung $\dot{\psi}+\dot{\beta}$ in (4.12) die Änderung der Sollbewegungsrichtung $v(t) \kappa_{s}\left(t^{\star}\right)$ verwendet:

$$
\dot{\psi}(t)+\dot{\beta}(t)=\kappa_{s}\left(t^{\star}\right) v(t) .
$$

Der Wert $\kappa_{s}\left(t^{\star}\right)$ ist dabei die Sollbahnkrümmung (2.13) aus der Trajektorienplanung für den zum Zeitpunkt $t$ gehörigen Parameter $t^{\star}$. Die Geschwindigkeit $v(t)$ ist die zum aktuellen Zeitpunkt gemessene Geschwindigkeit am Fahrzeugschwerpunkt. Der hintere Reifenschräglaufwinkel $\alpha_{h}$ ist eine Funktion der Gierwinkelgeschwindigkeit $\dot{\psi}(t)$, der Geschwindigkeit $v(t)$ und des Schwimmwinkels $\beta(t)$. Diese Größen werden über das integrierte Navigationssystem (Abbildung 3.9) bestimmt. Die in Gleichung (4.12) benötigte 
hintere Reifenseitenkraft $S_{h}(\dot{\psi}(t), v(t), \beta(t))$ wird über die Reifenkennlinie aus Abbildung 4.4 und den kinematischen Zusammenhang (4.7) ermittelt. Die Gleichung für die Berechnung der benötigten vorderen Reifenseitenkraft lautet damit:

$$
S_{v, f f}(t)=\frac{m v^{2}(t) \kappa_{s}\left(t^{\star}\right)-S_{h}(\dot{\psi}(t), v(t), \beta(t)) \cos \beta(t)}{\cos (\delta(t)-\beta(t))}
$$

Für Gleichung (4.14) wird der Lenkwinkel $\delta(t)$ benötigt. In der Regelungsstruktur (Abbildung 4.6) wird dieser über die Reifenkennlinie und die Fahrzeugkinematik aus der benötigten Reifenseitenkraft berechnet und ist daher für den aktuellen Abtastzeitpunkt noch nicht bekannt. Durch die hohe Abtastrate von $400 \mathrm{~Hz}$ kann für $\delta(t)$ jedoch der Lenkwinkel des letzten Abtastzeitpunktes verwendet werden, da die mögliche Änderung des Lenkwinkels zwischen zwei Abtastzeitpunkten der Messung gering ist. Die mit (4.14) berechnete Kraft $S_{v, f f}(t)$ ist die nominell benötigte vordere Reifenseitenkraft, um den Fahrzeugschwerpunkt bei dem aktuell gemessenen Fahrzeugzustand auf der durch $\kappa_{s}$ definierte Sollbahn zu halten. Aufgrund der Adaption mit Messgrößen besitzt die Vorsteuerung (4.14) eine Feedback-Struktur (vgl. Abbildung 4.6).

\section{PD-Regler}

Aufgrund von Modellunsicherheiten und Störeinflüssen muß der Vorsteuerungsanteil $S_{v, f f}(t)$ durch einen Regleranteil

$$
S_{v, f b}(t)=p_{d 1} d_{q}(t)+p_{d 2} \dot{d}_{q}(t)
$$

ergänzt werden, damit das Fahrzeug sich nicht immer weiter von der Sollbahn entfernt. Dieser Anteil hängt von der Querabweichung $d_{q}(t)$ in (2.14) des Fahrzeugschwerpunktes von der Sollbahn und der Annäherungsgeschwindigkeit $\dot{d}_{q}(t)$ in $(2.15)$ an die Sollbahn ab. Die zwei Regelparameter $p_{d 1}$ und $p_{d 2}$ werden wie für den Regler (4.10) mit Hilfe von Simulation und experimentell am realen Fahrzeug so bestimmt, daß sich das Fahrzeug der Sollbahn asymptotisch nähert. Simulationsuntersuchungen an agressiven Manövern wie zum Beispiel dem doppelten Spurwechselmanöver haben gezeigt (siehe Abschnitt 4.3), daß aufgrund des guten Verhaltens der Vorsteuerung der PD-Regler (4.15) zur Stabilisierung des Fahrzeugs entlang der Sollbahn ausreicht.

Die Parametrierung des PD-Reglers (4.15) für schnelle Fahrt läßt sich wie bei dem Regler für langsame Fahrt während einer Erprobung im Bedarfsfall vom Versuchsingenieur problemlos ändern. 


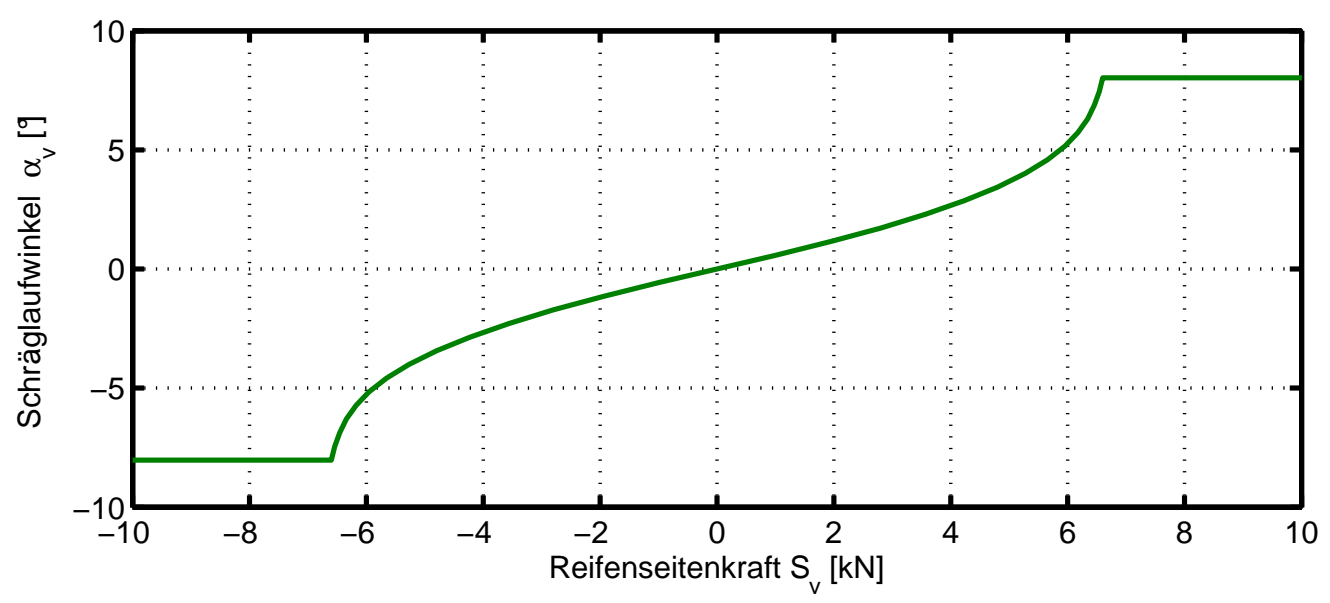

Abbildung 4.7: Inverse Reifenkennlinie $S_{v}^{-1}$ der Vorderräder mit Beschränkung des Schräglaufwinkels $\alpha_{v}$ im Maximum der Reifenseitenkraft $S_{v}$

\section{Inverse Lenkkinematik}

Nach Berechnung des Vorsteuerungsanteils $S_{v, f f}(t)$ in (4.14) und des Regelanteils $S_{v, f b}(t)$ in (4.15) sind die im Folgenden beschriebenen Rechenschritte

$$
S_{v, f f}, S_{v, f b} \stackrel{+}{\rightarrow} S_{v} \stackrel{S_{v}^{-1}}{\rightarrow} \alpha_{v} \stackrel{(4.6)}{\rightarrow} \delta \rightarrow \delta_{L 2}
$$

notwendig, um den für die Bahnfolge benötigten Lenkradwinkel $\delta_{L 2}$ für die Lenkmaschine zu ermitteln.

Im ersten Schritt wird die für die Bahnfolge nötige vordere Reifenseitenkraft

$$
S_{v}(t)=S_{v, f f}(t)+S_{v, f b}(t)
$$

ermittelt. Sie ist die Summe des Vorsteuer- und Regelanteils (4.14) und (4.15).

Im darauffolgenden Schritt muß der vordere Reifenschräglaufwinkel $\alpha_{v}$ ermittelt werden, der zu der erforderlichen Reifenseitenkraft $S_{v}(t)$ führt. Dafür muß die Reifenkennlinie aus Abbildung 4.4 invertiert werden. Die Reifenkennlinie ist nicht mit dem Reifenschräglaufwinkel monoton steigend. Die Reifenseitenkraft nimmt nach Erreichen eines Maximums bei weiterer Vergrößerung des Reifenschräglaufwinkels wieder ab. Für die Invertierung der Kennlinie (Abbildung 4.7) wird der Schräglaufwinkel auf den Wert beschränkt, an dem die Reifenseitenkraft ihr Maximum erreicht. Wird eine höhere Reifenseitenkraft angefordert, als die Reifen aufbringen können, wird der Reifenschräglaufwinkel $\alpha_{v}$ konstant auf dem Wert gehalten, der maximale Reifenseitenkraft hervorruft. In einem solchen Fall entsteht während der Fahrt im zeitlichen Verlauf des Soll-Lenkwinkels bei Erreichen der Reifensättigung ein Knick. Die abrupte Änderung der Lenkwinkelgeschwindigkeit kann durch eine reale Lenkmaschine nicht realisiert werden, was zu einem 
Überschwingen des Lenkwinkels führt. Die Auswirkungen auf die Fahrzeugbewegung sind jedoch gering, da kleine Variationen des Schräglaufwinkels an der Grenze der Reifenhaftung nur geringe Auswirkungen auf die resultierende Reifenseitenkraft haben.

Ist der für die Sollbahnkrümmung $\kappa_{s}\left(t^{\star}\right)$ benötigte vordere Reifenschräglaufwinkel $\alpha_{v}$ aus Abbildung 4.7 bekannt, wird im dritten Schritt der Lenkwinkel

$$
\delta(t)=\alpha_{v}\left(S_{v}(t)\right)+\arctan \left(\frac{l_{v} \dot{\psi}(t)+v(t) \sin \beta(t)}{v(t) \cos \beta(t)}\right)
$$

aus (4.6) berechnet. Der zweite Summand in (4.17) wirkt sich so aus, daß der gewünsche vordere Reifenschräglaufwinkel $\alpha_{v}\left(S_{v}(t)\right)$ unabhängig von der Fahrzeugbewegung eingestellt wird. Da die Fahrzeugbewegung $v(t), \dot{\psi}(t)$ und $\beta(t)$ durch die Messung bekannt ist und deren Auswirkung auf die Reifenseitekräfte direkt kompensiert wird, ist der gewünschte vordere Reifenschräglaufwinkel $\alpha_{v}$ unabhängig von der Gierbewegung des Fahrzeugs mit Gleichung (4.17) einstellbar. Durch die Rückkopplung des gemessenen Schwimmwinkels $\beta$ wirkt sich zum Beispiel ein Ausbrechen des Fahrzeughecks nicht auf den vorderen Schräglaufwinkel aus, solange nicht die Stellgrößenbeschränkungen für den realisierbaren Lenkwinkel erreicht werden. Die Stellgrößenbeschränkungen des Lenkwinkels sind durch die Lenkmaschine (maximale Lenkwinkelgeschwindigkeit) und das Lenkgestänge des Fahrzeugs (maximaler Lenkwinkel) bedingt.

Die Lenkmaschine wirkt aufgrund der Mechanik der Lenkung nur indirekt auf den Lenkwinkel $\delta$. Wird die Elastizität, Hysterese und veränderliche Untersetzung der Lenkmechanik vernachlässigt, hängt der Soll-Lenkradwinkel $\delta_{L 2}$ wie bereits erwähnt linear von dem Soll-Lenkwinkel $\delta$ ab. Dieser wird dem unterlagerten Regelkreis der Lenkmaschine übergeben, welcher für eine möglichst exakte Einhaltung des Soll-Lenkradwinkels verantwortlich ist.

\section{Zusammenfassung}

Die in diesem Abschnitt beschriebene Querregelung basiert auf dem dynamischen Fahrzeugmodell (4.4)-(4.7). Vergleicht man das Blockschaltbild der dynamischen Querregelung (Abbildung 4.6) mit dem der kinematischen (Abbildung 4.5), besteht der Unterschied sowohl in der Verwendung des gemessenen Fahrzeugzustandes für die Berechnung des Vorsteueranteils und der inversen Kinematik als auch in der Verwendung von Reifenkennlinien. Durch die Verwendung der Reifenkennlinien und die Einstellung des vorderen Reifenschräglaufwinkels mithilfe der Messung des aktuellen Fahrzeugzustandes wird eine Querregelung bis an die Grenzen der Reifenhaftung erreicht. 


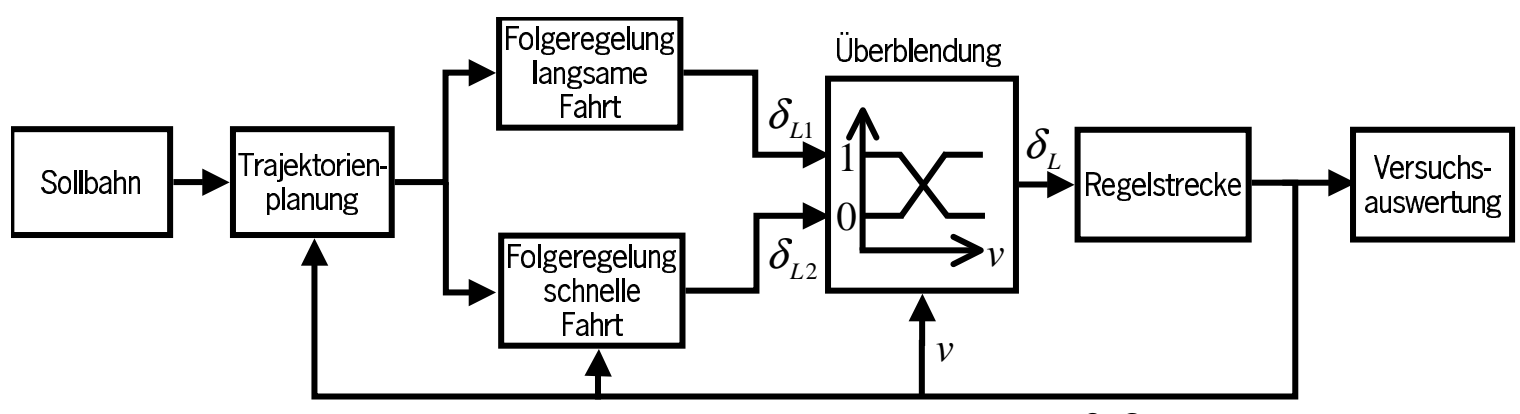

$(v, \beta, \delta, \psi, \dot{\psi}, \ldots)$

Abbildung 4.8: Geschwindigkeitsabhängiger Wechsel zwischen den Lenkradwinkeln $\delta_{L 1}$ und $\delta_{L 2}$ der Folgeregler im Bereich $20-30 \mathrm{~km} / \mathrm{h}$ mithilfe einer Überblendung für die Stellgröße $\delta_{L}$.

\subsubsection{Folgeregelung für alle Geschwindigkeiten}

In der realen Anwendung der Bahnfolgeregelung für geregelte Fahrdynamikmanöver betrifft die Folgeregelung für langsame Fahrt (Abschnitt 4.2.1) die Positionierung des Fahrzeugs auf dem Prüfgelände sowie den Anfahr- und Anhaltevorgang des durchzuführenden Manövers. Das eigentliche Fahrdynamikmanöver wird mit der Folgeregelung für schnelle Fahrt aus Abschnitt 4.2.2 realisiert. Daher ist ein Wechsel zwischen den beiden Folgeregelungen notwendig.

Die obere Geschwindigkeitsgrenze für die Folgeregelung auf Basis des kinematischen Fahrzeugmodells wurde mithilfe von Fahrversuchen auf $20 \mathrm{~km} / \mathrm{h}$ festgelegt. Sie ist ein Kompromiss zwischen der bei steigender Geschwindigkeit sinkenden Realitätstreue des kinematischen Modells (4.2) und der steigenden Genauigkeit der Schwimmwinkelmessung für die Folgeregelung auf Basis des dynamischen Fahrzeugmodells (4.4)-(4.7). Überschreitet das Fahrzeug diese Grenze, muß von der Folgeregelung für langsame Fahrt aus Abschnitt 4.2.1 auf die Folgeregelung für schnelle Fahrt aus Abschnitt 4.2.2 gewechselt werden. Um eine sprunghafte Änderung des Lenkradwinkels $\delta_{L}$ bei dem Wechsel zu vermeiden, wird für den Geschwindigkeitsbereich von $20-30 \mathrm{~km} / \mathrm{h}$ der Lenkradwinkel $\delta_{L 1}$ der Folgeregelung für langsame Fahrt auf den Lenkradwinkel $\delta_{L 2}$ der Folgeregelung für schnelle Fahrt übergeblendet (Abbildung 4.8). So wird der mögliche Geschwindigkeitbereich des Fahrzeugs für die Folgeregelung in folgende drei Bereiche aufgeteilt:

Bei geringen Geschwindigkeiten unterhalb $20 \mathrm{~km} / \mathrm{h}$ und der damit verbundenen Folgeregelung für langsame Fahrt aus Abschnitt 4.2.1 wird eine gute Bahnfolge bei gleichzeitig ruhigem Verlauf der Stellgröße $\delta_{L}$ erreicht. Das liegt daran, daß die für diese Folgeregelung benötigten Eingangsgrößen $\kappa_{s}$ aus der Trajektorienplanung glatt beziehungsweise $d_{q}$ und $\dot{d}_{q}$ aus dem Navigationssystem bei jeder Geschwindigkeit rauscharm sind.

In einem Übergangsbereich von 20-30km/h findet ein allmählicher Wechsel auf die Folgeregelung für schnelle Fahrt aus Abschnitt 4.2.2 statt. Da in diesem Geschwindigkeits- 

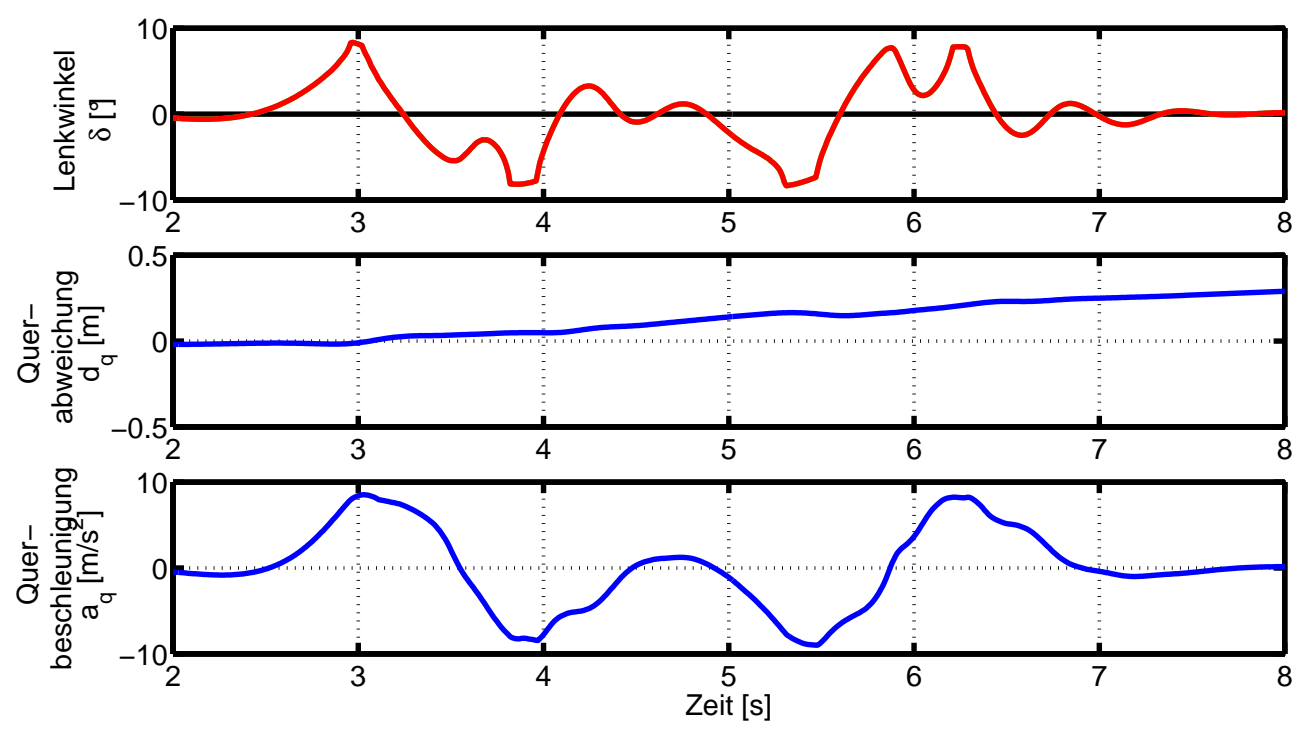

Abbildung 4.9: Simulation des Doppelspurwechsels mithilfe des detaillierten Fahrzeugmodells mit Vorsteuerung und ohne Regelung $\left(p_{d 1}=p_{d 2}=0\right)$

bereich bei kleinen Querbeschleunigungen die errechneten Lenkradwinkel $\delta_{L 1}$ und $\delta_{L 2}$ der beiden Folgeregler nahe beieinanderliegen, findet der Wechsel der Folgeregelung nahezu unmerklich statt.

Bei Geschwindigkeiten oberhalb $30 \mathrm{~km} / \mathrm{h}$ ist ausschließlich die Folgeregelung für schnelle Fahrt aktiv. Sie basiert auf dem dynamischen Fahrzeugmodell mit Reifenkennlinien und erreicht daher eine höhere Güte als die Regelung auf Basis des kinematischen Modells. Die dafür zusätzlich benötigte Messgröße des Schwimmwinkels $\beta$ ist bei Geschwindigkeiten oberhalb $30 \mathrm{~km} / \mathrm{h}$ ausreichend genau und rauscharm, um einen ruhigen Verlauf der Stellgröße $\delta_{L}$ zu erhalten.

\subsection{Simulation der Querregelung}

Zur simulationstechnischen Überprüfung der entworfenen Querregelung wird das validierte detaillierte Fahrzeugmodell eines Porsche Carrera verwendet, das in Abschnitt 4.1.3 bereits zur Bestimmung der Reifenkennlinien eingesetzt wurde. Als Testszenario dient ein Doppelspurwechsel, der durch schnelle Richtungswechsel mit hohen Querbeschleunigungen gekennzeichnet ist. Die Ortskurve der Sollbahn ist in Abbildung 2.2 auf Seite 12 dargestellt. Die für das Fahrzeug gewählte Einfahrgeschwindigkeit in das Manöver von ca. $60 \mathrm{~km} / \mathrm{h}$ wird vor Beginn der ersten Kurve durch den Versuchsingenieur eingestellt. Während des Manövers ist das Gaspedal in Leerlaufstellung, die Kupplung geschlossen und der 3. Gang eingelegt. 

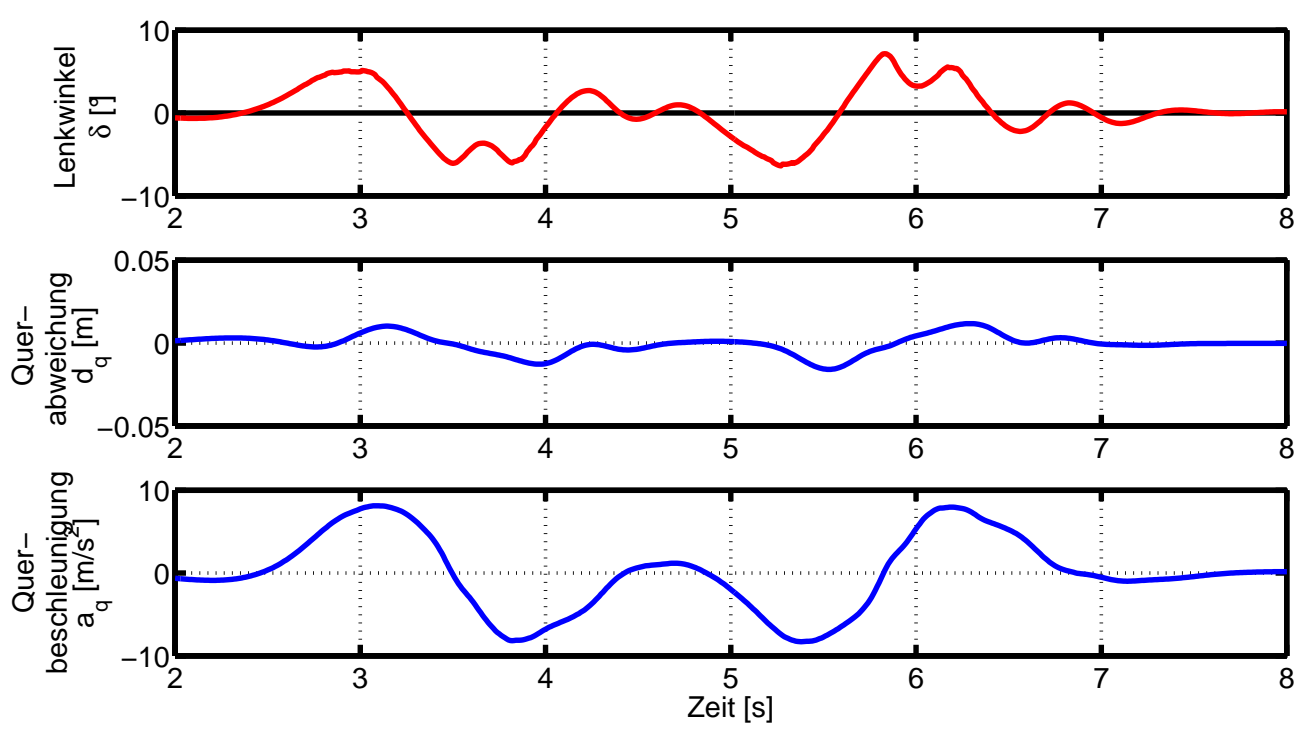

Abbildung 4.10: Simulation des Doppelspurwechsels mit Vorsteuerung und linearer PDRegelung

Während des in Abbildung 4.9 dargestellten Zeitausschnitts des Manövers ist aufgrund der Geschwindigkeit von ca. 60km/h ausschließlich die dynamische Querregelung aktiv. Um die Wirksamkeit der Vorsteuerung zu überprüfen, wird zunächst nur die Vorsteuerung (4.14) verwendet und die stabilisierende Regelung (4.15) deaktiviert $\left(p_{d 1}=p_{d 2}=0\right)$. Trotz der starken Vereinfachungen am dynamischen Entwurfsmodell für die Vorsteuerung und Vernachlässigung der Momentenbilanz (4.5) des Fahrzeugmodells zeigt die Bahn des Fahrzeugschwerpunktes eine große Ähnlichkeit mit der Sollbahn, was in Abbildung 4.9 an dem langsamen Anstieg der Querabweichung $d_{q}$ über der Zeit zu erkennen ist. Die Knicke im Verlauf des Lenkwinkels $\delta$ haben ihre Ursache in dem Knick der inversen vorderen Reifenkennlinie in Abbildung 4.7 bei Erreichen der maximalen Reifenseitenkraft. Eine weitere Erhöhung des Lenkwinkels würde in diesen Bereichen keine höhere Reifenseitenkraft zur Folge haben. Daß das simulierte Manöver an die Grenzen der Reifenhaftung heranreicht, ist auch an der maximalen Querbeschleunigung $a_{q}$ von etwa $1 \mathrm{~g}$ in Abbildung 4.9 zu erkennen. Die geringe Querabweichung des Fahrzeugschwerpunktes von der Sollbahn von ca. $30 \mathrm{~cm}$ am Ende des Doppelspurwechsels zeigt die Wirksamkeit der Vorsteuerung und der anschließender Berechnung des Lenkradwinkels mithilfe der inversen Lenkkinematik.

Im nächsten Schritt wird zusätzlich zu der Vorsteuerung die PD-Regelung für die Simulation des Doppelspurwechsels aktiviert. Als Folge entfällt das Wegdriften des Fahrzeugs von der Sollbahn und das simulierte Fahrzeug zeigt ein gutes Bahnfolgeverhalten (Abbildung 4.10).

Der Verlauf des Lenkwinkels $\delta$ in Abbildung 4.10 ist dem Verlauf von $\delta$ bei reiner Steue- 
rung aus Abbildung 4.9 ähnlich. Die in Bezug auf den Bahnverlauf häufigen Drehrichtungsänderungen des Lenkwinkels zeigen den Einfluß der für die Lenkwinkelberechnung verwendeten Messung des Fahrzeugzustandes. In den Bereichen maximaler Reifenseitenkraft hat der Regler allerdings einen signifikanten Anteil am Betrag des Lenkwinkels. Das liegt daran, daß in diesen Bereichen eine Änderung des Lenkwinkels nur geringe Auswirkung auf die Reifenseitenkraft hat. Der Regelanteil an der Reifenseitenkraft liegt dabei dennoch üblicherweise unter $15 \%$.

Durch die Regelung bleibt das Fahrzeug näher am geplanten glatten Sollbahnverlauf als bei reiner Steuerung. Daraus resultiert in Abbildung 4.10 ein etwas glatterer Verlauf der Querbeschleunigung $a_{q}$ als in Abbildung 4.9. Aus dem glatteren Querbeschleunigungsverlauf resultiert ein geringerer Bedarf von Reifenseitenkraft an den Maxima und somit größere Reserven für Korrekturen äußerer Störeinflüsse. Bemerkenswert an dem Ergebnis in Abbildung 4.10 ist besonders die trotz der hohen Querbeschleunigungen erreichte geringe Querabweichung (kleiner 5cm) des Fahrzeugschwerpunktes von der Sollbahn.

Die hier an einem Doppelspurwechsel und einem Porsche Carrera exemplarisch gezeigte Simulationsuntersuchung wurde auch für andere Fahrzeuggeschwindigkeiten und andere Fahrdynamikmanöver sowie mit dem Simulationsmodell eines Porsche Cayenne durchgeführt, um einen für das jeweils betrachtete Fahrzeug und alle in Frage kommenden Manöver verwendbaren Satz von Reglerparametern zu finden. Dabei wurde darauf geachtet, daß die Stabilität der Querregelung für die möglichen Testszenarien und für alternde Reifen sichergestellt ist.

\subsection{Zusammenfassung}

In diesem Kapitel wird die Querregelung für die Bahnfolge des Versuchsfahrzeugs beschrieben: Die Folgeregelung besteht aus einer nichtlinearen Vorsteuerung und einem linearen PD-Regler.

Für den Entwurf der Vorsteuerung werden zwei unterschiedliche Fahrzeugmodelle verwendet. Das erste ist ein kinematisches Fahrzeugmodell (Abschnitt 4.1.1), welches bei geringen Geschwindigkeiten bis zum Fahrzeugstillstand gültig ist. Es ist in seiner Formulierung geschwindigkeitsunabhängig und benötigt außer der Fahrwerksgeometrie keine weiteren Fahrzeugparameter. Das zweite Modell ist ein dynamisches Fahrzeugmodell (Abschnitt 4.1.2), welches den Reifenschlupf berücksichtigt und daher auch bei hohen Geschwindigkeiten und Querbeschleunigungen gültig ist. Für das dynamische Modell werden wegen der Berücksichtigung des Reifenschlupfes geeignete Reifenkennlinien benötigt. Zu deren Identifikation wird mit einem in der Porsche $A G$ vorhandenen detaillierten und validierten Modell des zu regelnden Fahrzeugs (wahlweise Porsche Carrera oder Porsche Cayenne) eine Simulation mehrerer stationärer Kreisfahrten durchgeführt. Auf diese Weise wird 
die bei Kurvenfahrt auftretende Veschiebung der vertikalen Reifenlast in Richtung der kurvenäußeren Räder in den Reifenkennlinien berücksichtigt.

Der Vorsteuerungsentwurf basiert auf dem kinematischen beziehungsweise dynamischen Fahrzeugmodell (Abschnitt 4.2). Für langsame Fahrt wird die Folgeregelung auf Basis des kinematischen Modells (Abschnitt 4.2.1) verwendet. Sie wird für das Anfahren und Anhalten sowie die Positionierung des Fahrzeugs auf dem Prüfgelände benötigt. Bei hohen Geschwindigkeiten und Querbeschleunigungen wird die Folgeregelung für schnelle Fahrt (Abschnitt 4.2.2) verwendet. Sie berücksichtigt die gemessene Fahrzeugbewegung bei der Berechnung des Lenkradwinkels und kann so im Rahmen der physikalischen Grenzen der Reifenhaftung auch ein ausbrechendes Fahrzeug entlang der vorgegebenen Bahn führen. Für den sanften Wechsel zwischen den beiden Folgereglern wird in dem Geschwindigkeitsbereich von $20-30 \mathrm{~km} / \mathrm{h}$ der berechnete Lenkradwinkel beider Folgeregler gemischt.

Zur Überprüfung der entworfenen Querregelung für die Bahnfolge des Versuchsfahrzeugs werden Simulationsuntersuchungen am Beispiel des doppelten Spurwechsels und anderen Manövern vorgenommen. So können die Parameter des linearen PD-Reglers bestimmt und die Stabilität des Regelkreises überprüft werden. Die Simulationsergebnisse zeigen eine hohe Wirksamkeit der Vorsteuerung und eine gute Bahnfolge auch bei Manövern bis an die physikalische Grenze der Reifenhaftung.

Aufgrund des positiven Ergebnisses der simulativen Überprüfung der Querregelung wird die beschriebene Bahnfolgeregelung implementiert und an einem Porsche Carrera und einem Porsche Cayenne erprobt. Die Implementierung der einzelnen Komponenten des Gesamtsystems der Querregelung und die Ergebnisse der Praxiserprobung werden in dem folgenden Kapitel beschrieben. 


\section{Kapitel 5}

\section{Implementierung und Erprobung der Bahnfolgeregelung}

Durch die simulative Überprüfung der entworfenen Querregelung in Abschnitt 4.3 sind alle Voraussetzungen gegeben, diese hard- und softwaretechnisch zu implementieren und im Fahrversuch praktisch zu erproben. Dazu müssen die Trajektorienplanung, das integrierte Navigationssystem und die Querregelung auf einer fahrzeugtauglichen Hardware echtzeitfähig realisiert werden. Dies beeinhaltet die Forderung nach einer geeigneten Stromversorgung, Beständigkeit gegen die im Fahrzeug vorkommenden mechanischen und thermischen Lasten, ausreichender Leistungsfähigkeit der Sensorik und Aktorik sowie ausreichender Rechenkapazität für die in den Kapiteln 2 bis 4 aufgeführten Berechnungschritte. Zusätzlich muß das System einfach bedienbar sein sowie das Speichern und die Auswertung der anfallenden Daten ermöglichen. Die Realisierung eines solchen Systems wird in Abschnitt 5.1 dieses Kapitels beschrieben.

Nach der Implementierung der Bahnfolgeregelung wird diese an zwei unterschiedlichen Fahrzeugen erprobt. Als Versuchsfahrzeuge werden ein Sportwagen vom Typ Porsche Carrera und ein sportlicher Geländewagen vom Typ Porsche Cayenne verwendet. Für die Erprobung steht das Prüfgelände der Porsche AG (Abbildung 1.3) in Weissach zur Verfügung. Im Bereich des Prüfgeländes befindet sich das sogenannte „Skid-Pad“, eine hindernisfreie, ebene Fahrdynamikfläche mit zwei Kreisbahnen von 60 und 200 Metern Durchmesser. In diesem Bereich können die Fahrversuche unter idealen Bedingungen durchgeführt werden. Die Durchführung und die Ergebnisse einer Auswahl von Versuchen werden in Abschnitt 5.2 beschrieben.

\subsection{Implementierung des Gesamtsystems}

Das vorhandene Porsche-Messsystem (Abbildung 5.1) erfüllt die Forderung nach Fahrzeugtauglichkeit und bietet die nötige Modularität und Flexibilität, um die entworfene 


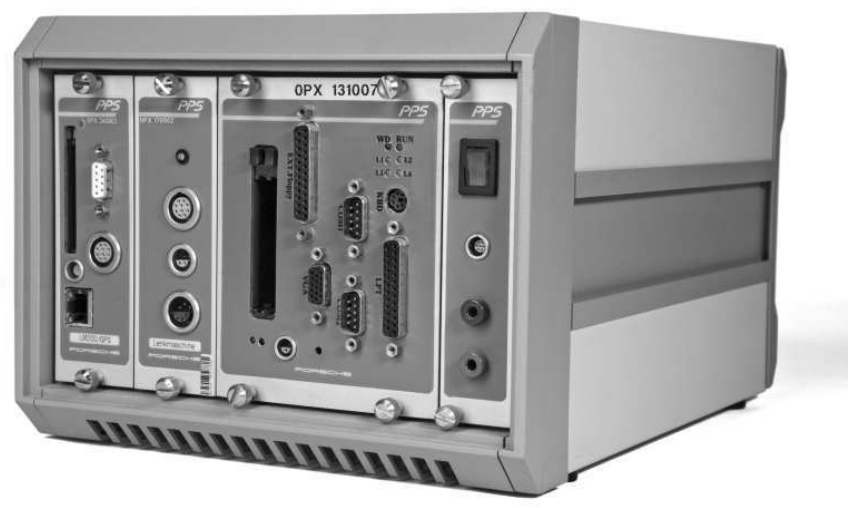

Abbildung 5.1: Navigations- und Reglermodul EMD315, Lenkmaschinen-Modul ELMA und PEA-Modul des Porsche-Messsystems in einem Modulrahmen mit parallelem Systembus und akkugepuffertem Netzteil

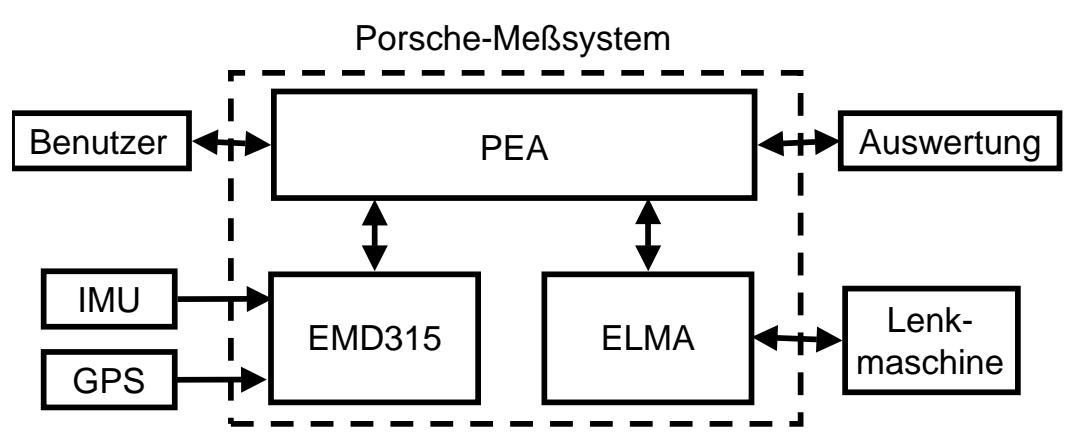

Abbildung 5.2: Schema der Implementierung der Bahnfolgeregelung mit Komponenten des Porsche Messsystems

Bahnfolgeregelung in die Praxis umsetzen zu können. Die Komponenten dieses Systems sind seit vielen Jahren im Fahrzeugeinsatz bewährt.

Das zugehörige Schema in Abbildung 5.2 zeigt den Aufbau der Bahnfolgeregelung mit den Komponenten des Porsche-Messsystems. Für die Datenaufzeichnung und die Bereitstellung der Bedienerschnittstelle wird ein $\mathrm{PEA}^{1}-$ Modul verwendet. Dieses enthält einen Industrie-PC mit Schnittstellen für ein Display und eine Tastatur und beinhaltet ein Programm für die Parametrierung der einzelnen Komponenten des Messsystems und den Messbetrieb. Für die Kommunikation mit diesen Einzelkomponenten sind Parametrierkommandos sowie Messkanäle und Sollwertkanäle mit einstellbarer Abtastrate definiert.

Als Plattform für die Trajektorienplanung, Messung der Fahrzeugbewegung und Querregelung für die Bahnfolge des Versuchsfahrzeugs dient das Prozessormodul EMD315² (Abbildung 5.3). Es besitzt neben den Schnittstellen für das inertiale Sensorpaket IMU

\footnotetext{
${ }^{1}$ PEA: Porsche Erfassung und Auswertung

${ }^{2}$ EMD315: Prozessormodul der Porsche Messtechnik
} 


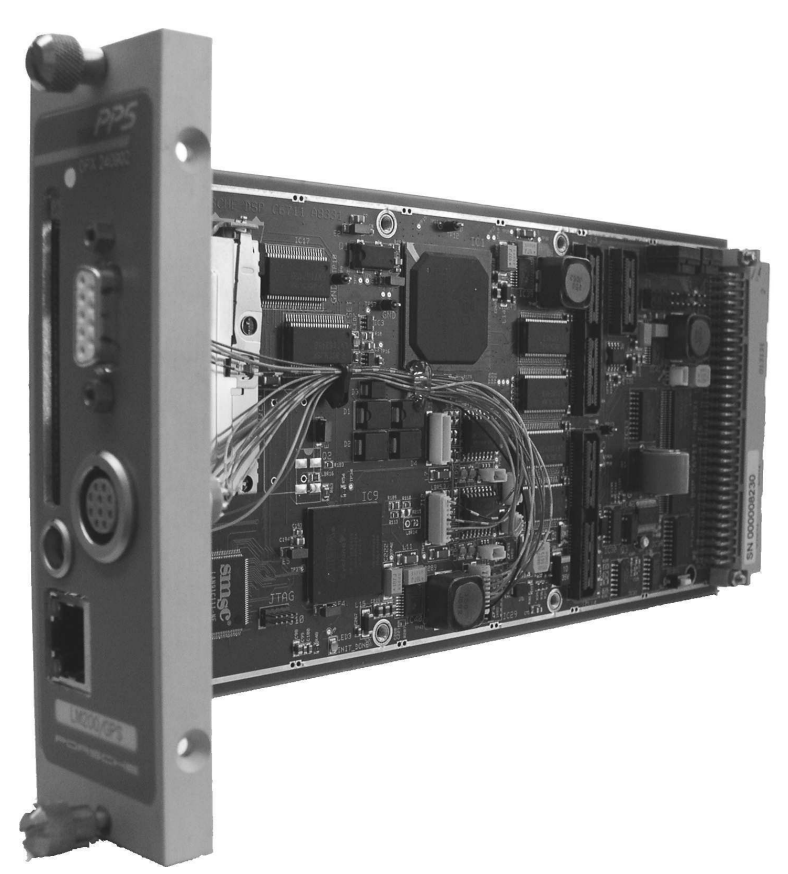

Abbildung 5.3: EMD315-Prozessormodul für das integrierte Navigationssystem mit Schnittstellen für einen GPS-Empfänger und ein Intertialsensorpaket sowie einem Speicherkartenschacht.

und den GPS-Empfänger einen Speicherkartenschacht. Für die notwendigen Berechnungen steht auf diesem Modul ein leistungsfähiger digitaler Signalprozessor von Texas Instruments zur Verfügung.

Die Messwerte des Navigationssystems und der Soll-Lenkradwinkel werden von dem EMD315-Modul über den Systembus des Porsche-Messsystems an das PEA-Modul gesendet. Dieses speichert die Daten und sendet den Soll-Lenkradwinkel an das Lenkmaschinen-Modul ELMA ${ }^{3}$ mit angeschlossener Lenkmaschine. Dieses wiederum sendet auf dem umgekehrten Weg den gemessenen Ist-Lenkwinkel an das EMD315-Modul.

Die Implementierung des Navigationssystems sowie der Trajektorienplanung und Querregelung auf einem einzigen EMD315-Moduls wird durch dessen Leistungsfähigkeit mit flexibel konfigurierbaren Schnittstellen und hoher Rechenleistung ermöglicht. Eine Übersicht des EMD315-Moduls ist in Abbildung 5.4 dargestellt. Die darin dargestellten funktionellen Blöcke für die Navigation mit der dazugehörigen Sensorik und die Regelung sind in den Abschnitten 5.1.1 und 5.1.2 beschrieben. Das in Abbildung 5.2 dargestellten ELMA-Modul für die Ansteuerung der Lenkmaschine wird zusammen mit der Lenkmaschine in Abschnitt 5.1.3 erläutert.

\footnotetext{
${ }^{3}$ ELMA: Elektrische Lenkmaschine
} 


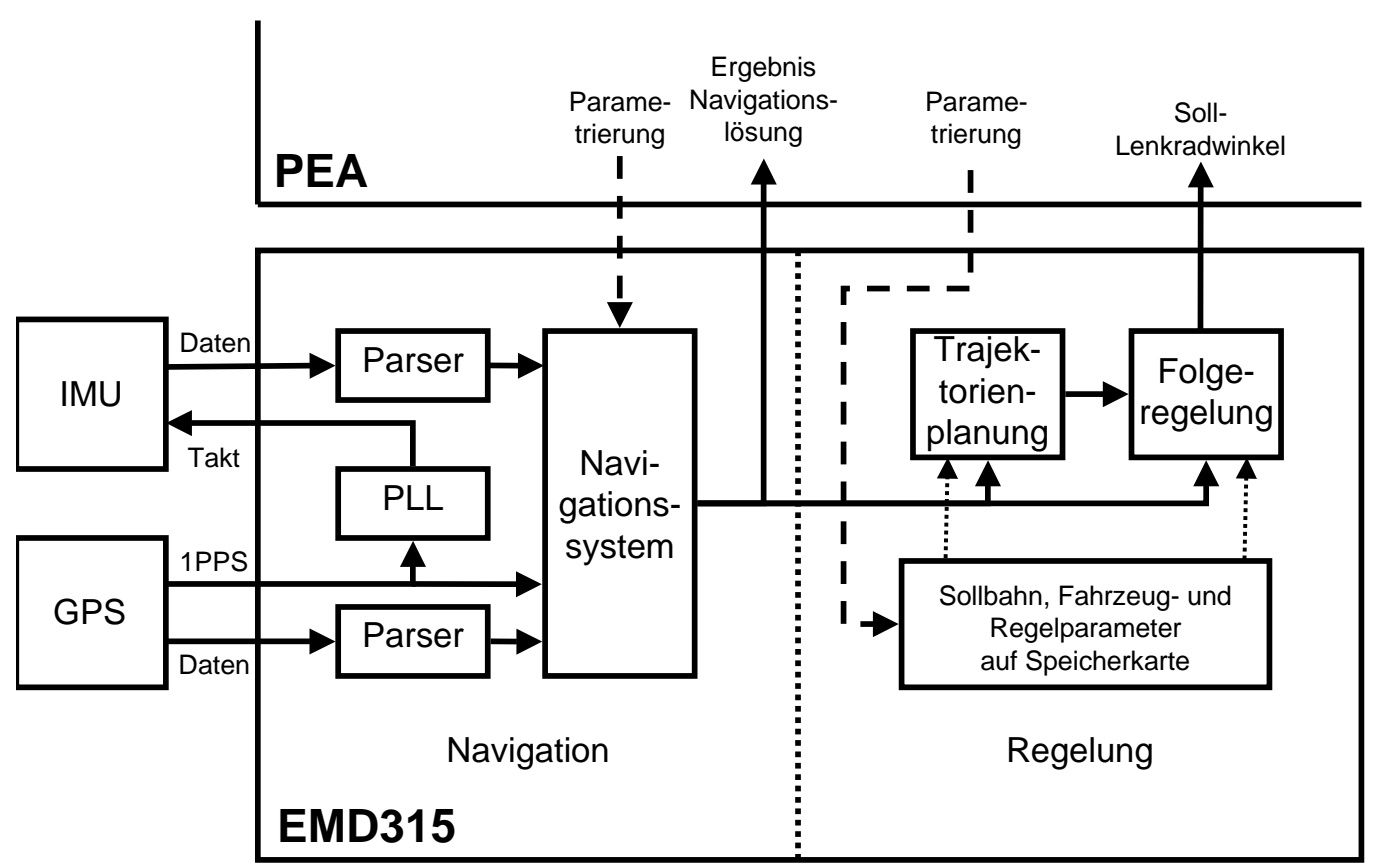

Abbildung 5.4: Schema des EMD315-Moduls mit Navigationssystem und Regelung mit Schnittstellen zu GPS und IMU sowie dem PEA-Modul in Abbildung 5.2

\subsubsection{Integriertes Navigationssystem}

Die Datenquellen für das integrierte Navigationssystem sind wie in Abbildung 5.4 dargestellt das inertiale Sensorpaket IMU und der Differential-GPS-Empfänger. Der GPSEmpfänger sendet die an der Antenne gemessene Position und Geschwindigkeit über eine serielle Schnittstelle an das EMD315-Modul. Die ankommenden Botschaften werden in einem Parser auf korrekte Prüfsumme überprüft und in die einzelnen Messwerte zerlegt. Zusätzlich hat der GPS-Empfänger einen Ausgang, welcher ein Taktsignal 1PPS synchron zu den Messzeitpunkten des Empfängers liefert. Dieser Takt dient als Eingang für eine PLL („phase locked loop“), welche daraus einen Takt von $400 \mathrm{~Hz}$ für das Inertialsensorpaket IMU erzeugt. So ist sichergestellt, daß die Messzeitpunkte des GPS-Empfängers und der Inertialsensoren synchronisiert sind. Mit jedem Taktimpuls wird von dem Inertialsensorpaket eine Botschaft mit den Messwerten erzeugt. Diese wird über einen Parser auf Korrektheit überprüft und in die Werte der einzelnen Sensorachsen zerlegt. Die beiden Parser und die PLL sind auf dem EMD315-Modul aus Echtzeitgründen teilweise in einem programmierbaren Logikbaustein untergebracht.

Am Ende der Sensordatenaufbereitung stehen dem Navigationssystem die Messergebnisse des Inertialsensorpaketes und des Differential-GPS-Empfängers zur Verfügung. Mit Hilfe des Taktsignals 1PPS werden alle Messwerte mit einem Zeitstempel versehen, um die korrekte Synchronisierung weiter zu gewährleisten. Für die Navigationsrechnung werden die Entfernungen der GPS-Antenne, des Inertialsensorpaketes und des Fahrzeug- 
schwerpunktes zueinander benötigt. Diese Informationen werden von dem übergeordneten PEA-Modul in Form eines Parametersatzes an das EMD315-Modul gesendet. Über den Parametersatz wird auch die gewünschte Dauer der automatischen Anfangsausrichtung festgelegt. Die Synchronisierung, die Navigationsrechnung sowie die Schätzung des Fehlers mittels Kalman-Filter erfolgen wie in Abbildung 3.9 skizziert und wie in Kapitel 3 beschrieben.

Das Ergebnis des integrierten Navigationssystem liegt im EMD315-Modul intern mit einer Datenrate von $400 \mathrm{~Hz}$ zur Weiterverarbeitung durch die Regelung vor und wird gleichzeitig in Form von Messkanälen an das PEA-Modul gesendet. So ist eine Aufzeichnung der Bewegung des Fahrzeugs für die spätere Analyse zur Beurteilung des Fahrverhaltens möglich. Dadurch kann das EMD315-Modul auch als reines Navigationssystem für jede Art von Versuchen wie zum Beispiel manuell oder gesteuert durchgeführte Versuche eingesetzt werden.

\section{Schwimmwinkelkorrektur}

Die Schwimmwinkelmessung kann nach der Anfangsausrichtung des integrierten Navigationssystems aufgrund der langsamen Konvergenz des Navigationsfilters für den Fehlerzustand des Gierwinkels fehlerbehaftet sein (Abschnitt 3.2.2). Bei der Fahrdynamikerprobung treten jedoch immer wieder Phasen langsamer kinematischer Fahrt auf. Befindet sich das Fahrzeug in einer solchen Phase ohne Reifenschlupf, läßt sich der Schwimmwinkel aus dem kinematischen Fahrzeugmodell berechnen und die Differenz zur Messung beseitigen. Diese Korrektur wird immer dann berechnet, wenn das Fahrzeug bei sehr geringer Querbeschleunigung (kleiner 0,1g) der Sollbahn sehr eng $\left(d_{q}<5 \mathrm{~cm}\right)$ folgt. Dadurch ist die Bahnkrümmung genau bekannt und somit auch der zu erwartende Schwimmwinkel.

\subsubsection{Trajektorienplanung und Querregelung}

Die Ergebnisdaten des integrierten Navigationssystems liegen innerhalb des EMD315-Moduls (Abbildung 5.4) in Echtzeit für die Trajektorienplanung und Folgeregelung vor. Da die Berechnungen für die Bahnfolgeregelung auf dem selben digitalen Signalprozessor wie die Navigationsrechnung stattfinden, entsteht keine nennenswerte Verzögerung bei der Datenübermittlung. Die Trajektorienplanung verarbeitet die Position, Geschwindigkeit und Bewegungsrichtung des Fahrzeugschwerpunktes. Um daraus wie in Abschnitt 2.3 beschrieben die Sollposition sowie die Regelabweichung zu bestimmen, wird die Sollbahn (2.7) benötigt. Diese wird wie in Abschnitt 2.2 dargestellt in Form ihrer Polynomkoeffizienten $a_{x i k}$ und $a_{y i k}$ der Splinesegmente (2.5) und (2.6) auf einer wechselbaren Speicherkarte zur Verfügung gestellt. Die verschiedenen Sollbahnen sind hierbei in jeweils einer Datei abgelegt und können über die Benutzerschnittstelle des PEA-Moduls ausgewählt beziehungsweise parametriert werden. 
Genau wie die Sollbahn kann auch das verwendete Fahrzeug mithilfe der Benutzerschnittstelle des PEA-Moduls parametriert werden. Die zu jedem Fahrzeug gehörige Datei beinhaltet die Länge des Fahrzeugs, die Lage des Fahrzeugschwerpunkts, die Lenkkinematik und die Reifenkennlinien. Zusätzlich sind für jedes Fahrzeug die Parameter der beiden PD-Regler (4.10) und (4.15) für langsame beziehungsweise schnelle Fahrt (Abschnitt 4.2) hinterlegt. Für die Praxiserprobung der Bahnfolgeregelung liegen Dateien mit den Fahrzeug- und Regelparametern der Fahrzeuge Porsche Carrera und Porsche Cayenne vor. Mithilfe dieser Informationen, der Ausgabe der Trajektorienplanung und dem Ergebnis des integrierten Navigationssystems werden entsprechend Abschnitt 4.2 die Berechnungen für die Folgeregelung durchgeführt. Die Frequenz der Neuberechnung entspricht der Datenrate des Navigationssystems von 400Hz. Die Ausgabe der Folgeregelung, der Soll-Lenkradwinkel $\delta_{L}$, wird dem PEA-Modul genau wie die Ergebnisse der Navigationsrechnung in Form eines Messwertkanals übergeben.

\subsubsection{Lenkmaschine}

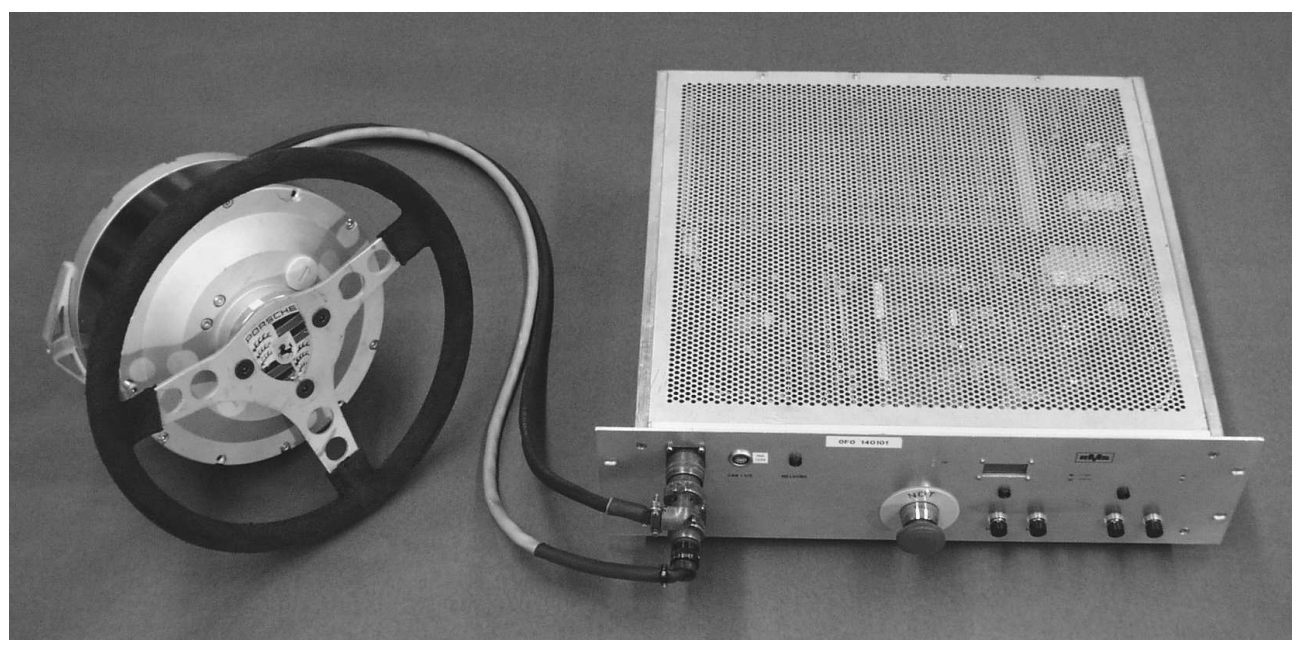

Abbildung 5.5: Elektrische Lenkmaschine der Firma RMS Testsystems, bestehend aus einem Lenkmotor und dem dazugehörigen Steuergerät

Als Aktor für den Lenkradwinkel dient die in Abbildung 5.5 dargestellte Lenkmaschine der Firma RMS Testsystems. Sie besteht aus einem Elektromotor mit integriertem Drehwinkelencoder und dem dazugehörigen Steuergerät. Die maximale Winkelgeschwindigkeit dieses Systems beträgt $800^{\circ}$ / s bei einem Lenkmoment von $150 \mathrm{Nm}$. Die Welle des Motors wird direkt anstelle des Serienlenkrads an der Lenksäule des Fahrzeugs montiert. Um ein manuelles Lenken des Fahrzeugs zu ermöglichen, ist ein Lenkrad an der durchgehenden Welle des Motors befestigt. So kann bei stromlosem Motor das Fahrzeug herkömmlich gelenkt und manuell auf das Prüfgelände gefahren werden. Damit 
der Motor ein Lenkmoment übertragen kann, muß der Stator des Motors über Streben an der Karosserie des Fahrzeugs abgestützt werden. Das Lenkmaschinen-Steuergerät erhält den Soll-Lenkradwinkel über den CAN-Bus ${ }^{4}$, ein in der Automobilbranche weitverbreites asynchrones und serielles Bussystem. Mithilfe des in den Motor integrierten Drehwinkelencoders stellt das Lenkmaschinen-Steuergerät den übermittelten absoluten Soll-Lenkradwinkel ein.

Die Kommunikation für die Übermittlung des Soll-Lenkradwinkels geschieht im Verbund mit dem PEA-Modul mithilfe des in Abbildung 5.1 gezeigten ELMA-Moduls. Dieses Modul erhält den Soll-Lenkradwinkel als Sollwertkanal von dem PEA-Modul und leitet ihn über den CAN-Bus an das Lenkmaschinen-Steuergerät weiter. Weiter sorgt das ELMAModul für die Initialisierung und Überwachung der Lenkmaschine. Für die Bedienung der Lenkmaschine bei gesteuert durchgeführten Manöver existiert für die gesteuerten Fahrversuche bereits ein Bediengerät mit Statusanzeige, Start- und Stop-Taste sowie einem Not-Aus-Schalter. Dieses wird auch für die Bedienung mit Bahnfolgeregelung verwendet. Ist das System über die Bedienoberfläche des PEA-Moduls in Messbereitschaft versetzt, wird der Soll-Lenkradwinkel bei Betätigung der Start-Taste an das LenkmaschinenSteuergerät weiter geleitet. Bei Betätigung der Stop-Taste werden die Motorwicklungen stromlos geschaltet. Wird der Not-Aus-Schalter betätigt, wird die elektrische Verbindung zu dem Motor aufgetrennt. Bei stromlosem Motor hat der Fahrer lediglich das zusätzliche Massenträgheitsmoment des Motorläufers zu überwinden und kann das Fahrzeug ansonsten normal lenken. Da die Bahnfolgeregelung ausschließlich über den Lenkradwinkel Einfluß auf das Fahrzeug nimmt und immer ein Fahrer den Antriebsstrang und die Bremsanlage kontrolliert, ist eine sofortige Übername der Fahrzeugkontrolle durch den Fahrer im Fall einer Systemstörung möglich.

\subsubsection{Benutzerschnittstelle und Datenaufzeichnung}

Die Benutzerschnittstelle des PEA-Moduls ermöglicht die Parametrierung des Gesamtsystems. In der Parametrierung für das Navigationssystem auf dem EMD315-Modul wird die Entfernung der GPS-Antenne zu dem Inertialsensorpaket und dessen Abstand zu dem Fahrzeugschwerpunkt angegeben. Weitere Einstellmöglichkeiten betreffen die Dauer der Anfangsausrichtung des Navigationssystems sowie den Typ des angeschlossenen GPS-Empfängers und Inertialsensorpakets. Die gewählten Parameter werden in einem nichtflüchtigen Speicher des EMD315-Moduls abgelegt. Ist das Navigationssystem parametriert, werden beim Einschalten der Stromversorgung das Inertialsensorpaket und der GPS-Empfänger initialisiert. Nach Abschluss der Sensorinitialisierung beginnt die automatische Anfangsausrichtung des Navigationssystems. Bis zum Abschluss der Anfangsausrichtung darf das Fahrzeug nicht bewegt werden. Nach erfolgter Anfangsausrichtung

\footnotetext{
${ }^{4} \mathrm{CAN}$ : Controller Area Network
} 
wird die Navigationsrechnung bis zum Ausschalten der Stromversorgung unterbrechungsfrei durchgeführt und benötigt die Zeit für die Initialisierung und Anfangsausrichtung (ca. 3 Minuten) daher nur einmalig während der Aktivierung des gesamten Mess- und Regelaufbaus.

Die Folgeregelung auf dem EMD315-Modul erhält von dem PEA-Modul als Parameter den Namen der Sollbahn und den Fahrzeugtyp. Die beiden Parameter bestimmen, aus welchen Dateien auf der Speicherkarte im EMD315-Modul die Sollbahn und die Fahrzeugund Regelparameter ausgelesen werden. Diese Dateien werden vor den Fahrversuchen angelegt und sollen im Fahrzeug nicht verändert werden.

Für die Parametrierung des Lenkmaschinen-Moduls ELMA wird der Name des Sollwertkanals für den Lenkradwinkel benötigt. Dieser wird dann von dem PEA-Modul kontinuierlich mit der maximalen Datenrate von $100 \mathrm{~Hz}$ an das Lenkmaschinen-Steuergerät gesendet.

Für die Auswertung der Versuche soll das Ergebnis der Navigationsrechnung aufgezeichnet werden. Für jedes Modul in dem Porsche-Messsystem (Abbildung 5.2) kann die Datenrate vorgegeben werden, mit der die ausgewählten, für die spätere Auswertung benötigten Messwertkanäle aufgezeichnet werden. Zusätzlich zu den Ergebniskanälen des Navigationssystems kann das in Abbildung 5.1 dargestellte System für die Bahnfolgeregelung bei Verwendung eines breiteren Systemrahmens um Module und Messkanäle für die Messung weiterer physikalischer Größen am Fahrzeug wie zum Beispiel Temperaturen oder Drücke erweitert werden.

Der Bedienungsablauf für ein einzelnes bahngeregeltes Fahrdynamikmanöver beginnt mit der Aktivschaltung des Messsystems. Von da an werden die Messkanäle der Messmodule von dem PEA-Modul abgefragt und auf einem Bildschirm im Fahrzeug angezeigt. Anschließend aktiviert der Versuchsingenieur die Messung. Zu diesem Zeitpunkt wird eine neue Datei auf dem Datenträger des PEA-Moduls angelegt und die in der Parametrierung ausgewählten Messkanäle mit der vorgegebenen Datenrate aufgezeichnet. Die Trajektorienplanung und die Folgeregelung des EMD315-Moduls berechnen den SollLenkradwinkel. Drückt nun der Versuchingenieur die Start-Taste des Bediengeräts der Lenkmaschine, wird der Soll-Lenkradwinkel an das Lenkmaschinen-Steuergerät weitergeleitet und durch den Motor der Lenkmaschine realisiert. Nach Beendigung des bahnfolgegeregelten Versuchs betätigt der Versuchsingenieur die Stop-Taste an dem Bediengerät der Lenkmaschine und kann das Fahrzeug von diesem Zeitpunkt an wieder manuell lenken. Die Messung wird über die Bedienoberfläche des PEA-Moduls beendet und die dazugehörige Datei auf dem Datenträger geschlossen. Nach Durchführung der Versuche werden die Messungen vom Datenträger des PEA-Moduls auf einen Büro-PC kopiert und dort ausgewertet. 


\subsection{Versuchsergebnisse}

Das Ergebnis der Simulationsuntersuchung am Beispiel des Doppelspurwechsels mit einem Porsche Carrera aus Abschnitt 4.3 soll hier exemplarisch validiert werden. Dazu wird dasselbe Fahrdynamikmanöver im Praxisversuch mit einem Porsche Carrera [26] wie in der Simulation durchgeführt. Zusätzlich wird die Bahnfolgeregelung des Doppelspurwechselmanövers an einem Porsche Cayenne [27] erprobt. Die Sollbahnvorgabe ist mit der Vorgabe für die Simulationsuntersuchung identisch. Auch die Fahrzeugparameter wie Masse, Schwerpunktlage, Reifenkennlinien sowie die Regelparameter der Folgeregelung werden für den Praxisversuch übernommen.

In den folgenden Abschnitten werden einige Versuchsergebnisse für die beiden Fahrzeuge Porsche Carrera und Porsche Cayenne diskutiert. Die Versuchsaufbauten im jeweiligen Fahrzeug sind sehr ähnlich und werden daher nur für den Porsche Carrera vorgestellt.

\subsubsection{Doppelspurwechsel}

Die im Folgenden dargestellten Versuchsergebnisse stammen aus geregelt durchgeführten Doppelspurwechselmanövern. Die Erprobung wird auf dem Skid-Pad des Prüfgeländes Weissach (Abbildung 1.3) durchgeführt. Dort herrscht in alle Richtungen freie Sicht auf den Horizont und somit ist jederzeit einwandfreier GPS-Empfang möglich. Die DGPSBasisstation befindet sich am Rande der Strecke. Die Entfernung zwischen Fahrzeug und Basisstation beträgt wenige hundert Meter. Daher liegen ideale Empfangsbedingungen für das integrierte Navigationssystem vor.

Die in das Luftbild des Skid-Pads in Abbildung 5.6 eingezeichnete Sollbahn für den Spurwechsel hat denselben Verlauf wie in der Simulation. An das Doppelspurwechselmanöver schließt eine halbkreisförmige Sollbahn an, die das bahngeregelte Fahrzeug auf einem großen Halbkreis des Skid-Pads mit Radius 100m an den Startpunkt des Doppelspurwechselmanövers auf der Mittelgeraden zurückbringt. Die Fahrtrichtung ist gegen den Uhrzeigersinn gerichtet.

An der eingezeichneten Sollbahn ist links ein zu Beginn der Fahrt geplanter Spline (Abschnitt 2.4.1) zu erkennen. An dieser Stelle befindet sich die Zufahrt des Skid-Pads. Für die Versuche wird das Fahrzeug manuell auf das Skid-Pad gefahren und an der Einfahrt geparkt. Dort wird das Messsystem eingeschaltet und die Initialisierung abgewartet. Nach Aktivierung der Regelung wird automatisch eine Bahn, die zu der eigentlichen Sollbahn auf der Mittelgeraden führt, geplant. Die Sollbahn wird zur Kontrolle der Fahrbahn und der korrekten Funktion des Mess- und Regelsystems einmal langsam befahren, um anschließend die folgenden Runden mit höherer Geschwindigkeit absolvieren zu können.

Wie in Abbildung $5.6 \mathrm{zu}$ erkennen ist, gibt es für das Doppelspurwechselmanövers im Zentrum des Skid-Pads ausreichend freie Fläche, um die Versuche sicher durchführen zu 


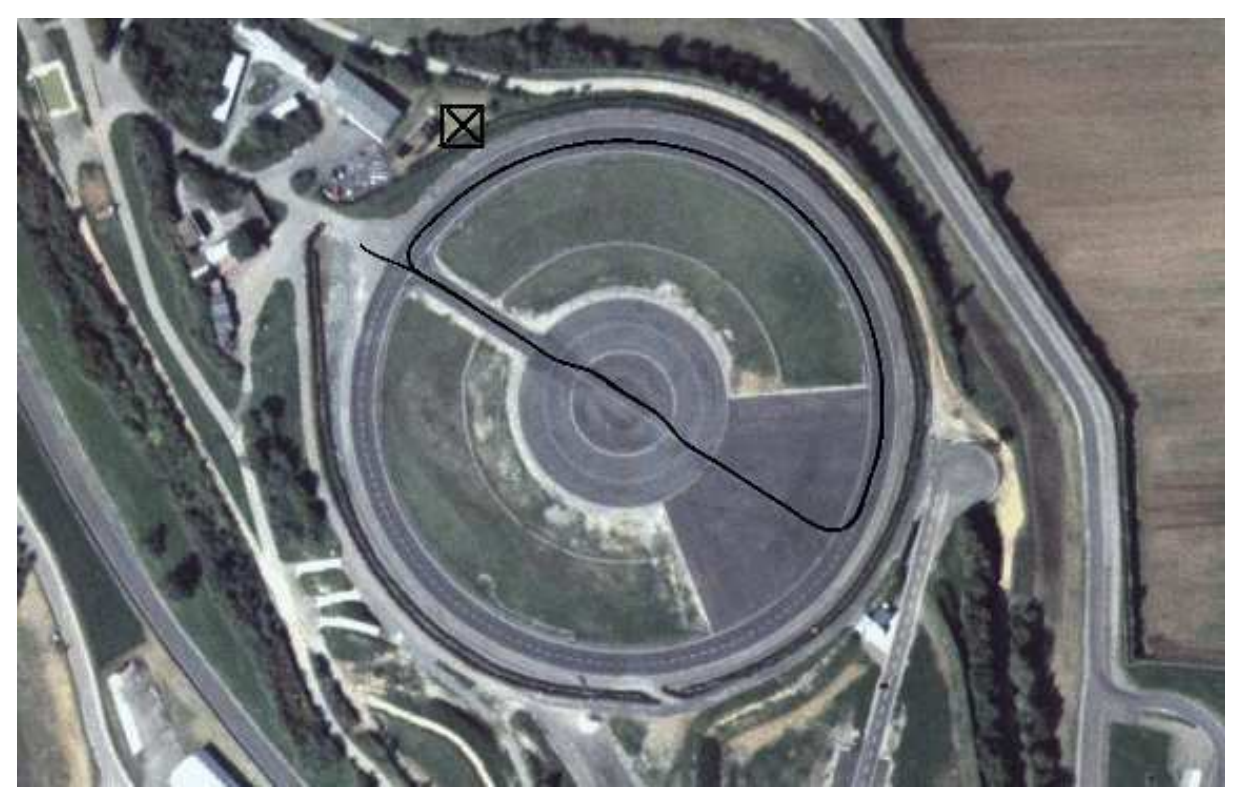

Abbildung 5.6: Geschlossene Sollbahn des Doppelspurwechselmanövers und Position der DGPS-Basisstation auf dem Skid-Pad des Prüfgeländes der Porsche AG in Weissach (Bild: Google Earth)

können. Sollte das Fahrzeug bei Überschreitung der physikalischen Grenze der Reifenhaftung dennoch die asphaltierte Fläche verlassen, befinden sich in den Randbereichen geschotterte Auslaufzonen und Reifenstapel.

\subsubsection{Versuchsfahrzeug Porsche Carrera}

Für eine umfangreiche experimentelle Erprobung steht ein Fahrzeug vom Typ Porsche Carrera zur Verfügung (Abbildung 5.7). Es handelt sich um einen Sportwagen mit tief liegendem Schwerpunkt, Heckmotor und Hinterradantrieb [26]. Für den Einbau des Messund Regelsystems wird der Beifahrersitz entfernt und stattdessen ein Aufbau aus Aluminiumprofilen an den Sitzschienen befestigt (Abbildung 5.8). Auch das inertiale Sensorpaket wird mit den Schienen der Sitzbefestigung und damit der Karosserie fest verschraubt. Das EMD315-Modul mit dem Regel- und Navigationssystem sitzt zusammen mit dem Lenkmaschinen-Modul ELMA und dem PEA-Modul für die Messdatenaufzeichnung in einem Rahmen des Porsche Messsystems. Dabei ist die Funktionalität mit dem in Abbildung 5.1 gezeigten Messsystem identisch. Darüber befindet sich das Lenkmaschinen-Steuergerät. Das Moment des Elektromotors wird über eine Strebe an dem Messtechnik-Aufbau auf der Beifahrerseite abgestützt. In der Abbildung nicht zu erkennen sind der GPS-Empfänger und das Funkmodem für den Empfang der DifferentialGPS-Informationen. Die GPS-Antenne befindet sich mittig auf dem Fahrzeugdach (Abbildung 5.7). 


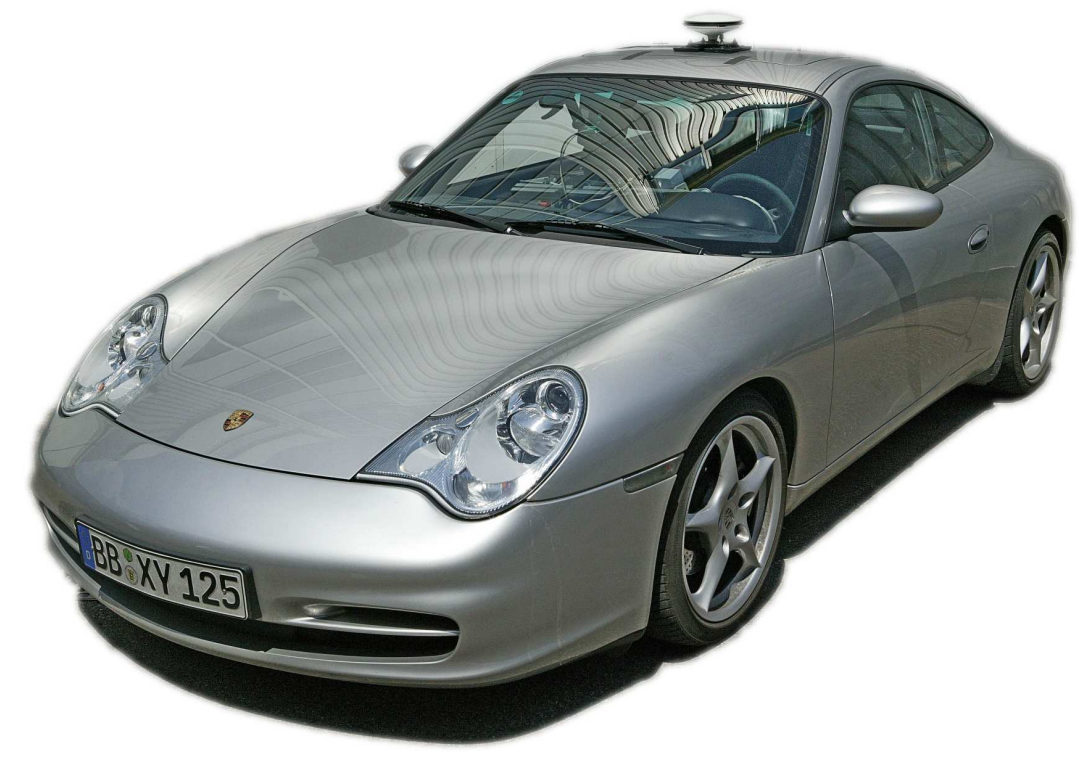

Abbildung 5.7: Außenansicht des für die Erprobung ausgerüsteten Versuchsfahrzeugs vom Typ Porsche Carrera (Bild: Porsche AG)

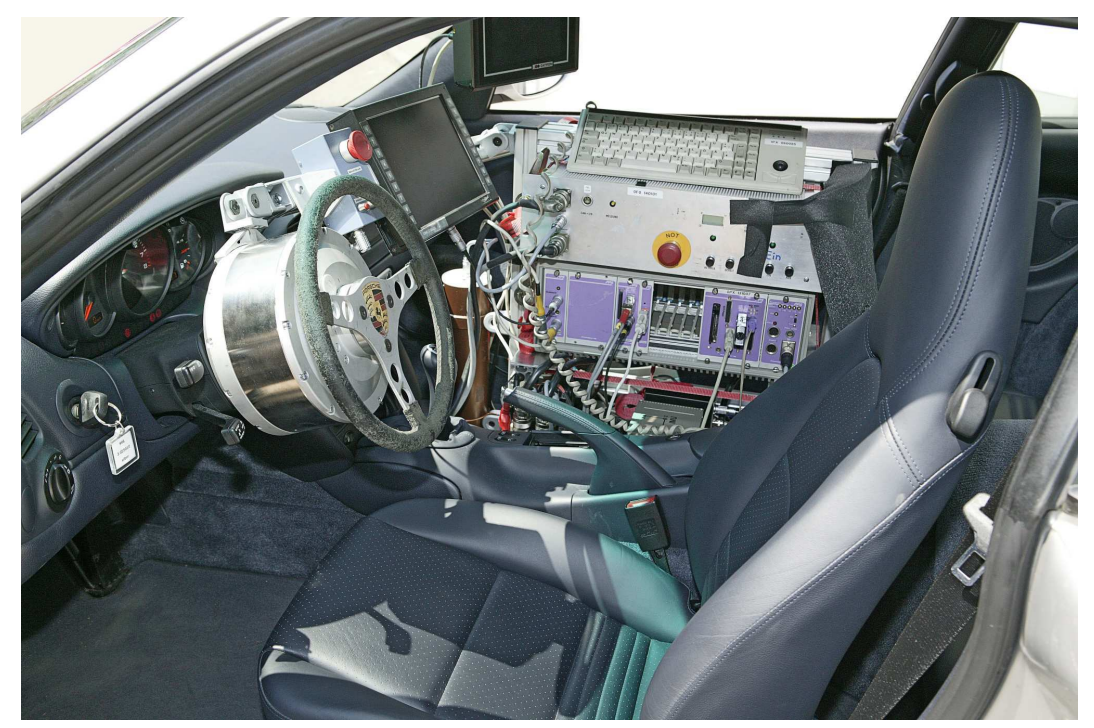

Abbildung 5.8: Innenraum des Versuchsfahrzeugs vom Typ Porsche Carrera mit dem Regelsystem auf Basis des Porsche Messsystems und mit der Lenkmaschine (Bild: Porsche $A G)$ 

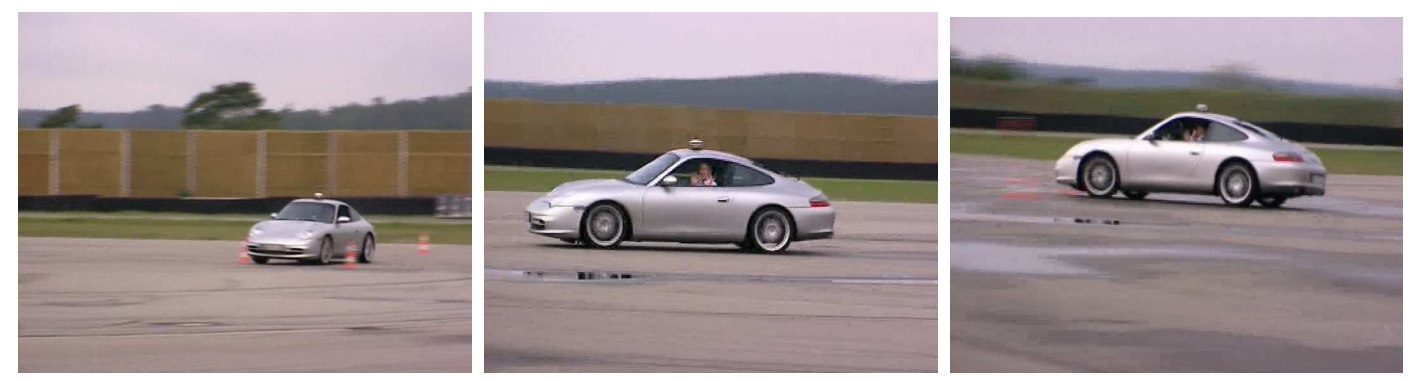

Abbildung 5.9: Versuchsfahrzeug Porsche Carrera während eines geregelten Doppelspurwechsels auf dem Skid-Pad in Weissach (Bild: Porsche AG)

\subsubsection{Versuchsergebnisse Porsche Carrera}

Für die praktische Überprüfung der Bahnfolgeregelung wird das Doppelspurwechselmanöver zuerst mit einer Geschwindigkeit zwischen 10 und 20km/h durchfahren, um die Folgeregelung für langsame Fahrt aus Abschnitt 4.2.1 zu verwenden. Die Messergebnisse $\mathrm{zu}$ diesem Versuch sind in Abbildung 5.10 dargestellt.

Im ersten Diagramm von oben ist die Position des Fahrzeugschwerpunktes in der $(x, y)$ Ebene dargestellt. Die Bahn ist identisch mit der Sollbahn in Abbildung 2.2. Für eine platzsparende Darstellung ist das Koordinatensystem gegenüber der realen Lage (siehe Abbildung 5.6) so gedreht, daß die Hauptachse des Doppelspurwechselmanövers parallel zu der $x$-Achse liegt. Die Skalierung der $x$-Achse ist nicht äquidistant, sondern so gewählt, daß sie mit der Zeitachse der darunterliegenden Diagramme übereinstimmt. Das Fahrzeug bewegt sich in dieser Darstellung von links nach rechts.

Das zweite Diagramm zeigt die Geschwindigkeit $v(t)$ für den 30-sekündigen Ausschnitt der Messung. Während des Spurwechselmanövers wurde die Geschwindigkeit zwischen 15 und $18 \mathrm{~km} / \mathrm{h}$ gehalten, so daß entsprechend Abschnitt 4.2.3 ausschließlich der Folgeregler für langsame Fahrt aus Abschnitt 4.2.1 verwendet wird.

Das dritte Diagramm zeigt den Lenkradwinkel $\delta_{L}(t)$ der Lenkmaschine. Er ist nach Gleichung (4.9) direkt abhängig von der Sollbahnkrümmung $\kappa_{s}$.

Mit diesem Lenkwinkel folgt der Fahrzeugschwerpunkt der Sollbahn mit einem Querabstand $d_{q}(t)$, der in dem vierten Diagramm dargestellt ist. Der Querabstand bleibt in diesem Versuch jederzeit kleiner als $5 \mathrm{~cm}$. Der vorhandene Fehler wird hauptsächlich durch die nicht exakt lineare Kennlinie das Lenkgetriebes hervorgerufen.

Die gemessene horizontierte Querbeschleunigung $a_{q}(t)$ im fünften Diagramm ist gering und rechtfertigt die Regelung auf Basis des kinematischen Fahrzeugmodells (4.2).

In einem zweiten Versuch wird der Doppelspurwechsel mit einer größeren Geschwindigkeit gefahren, die das Fahrzeug an die physikalische Grenze der Reifenhaftung bringt. Es wird 

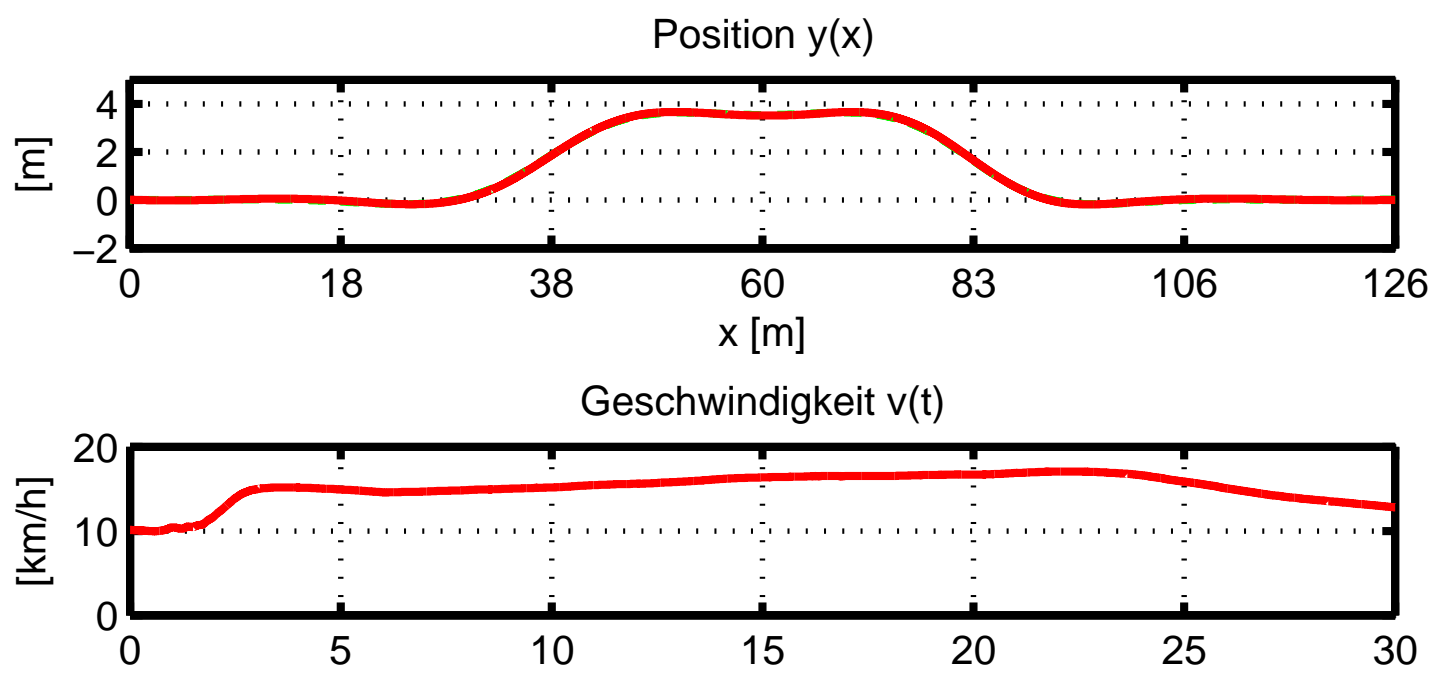

Lenkradwinkel $\delta_{L}(t)$

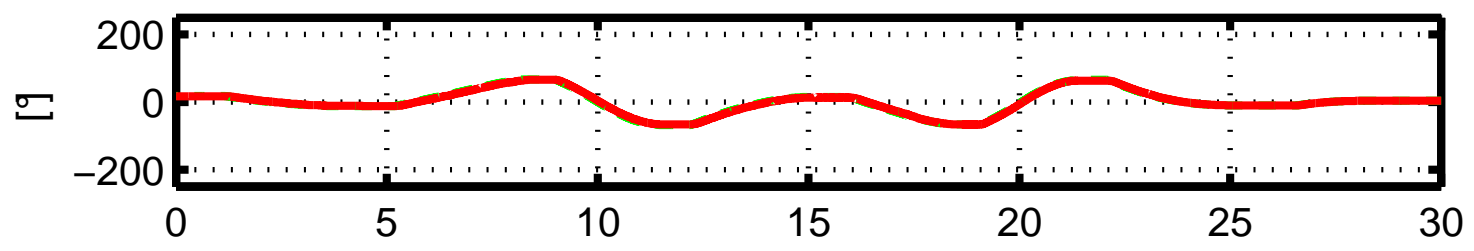

Querabstand $d_{q}(t)$

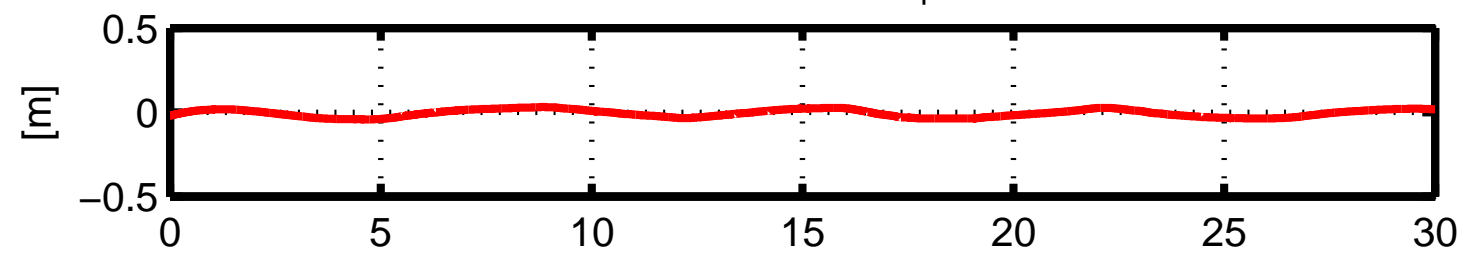

Querbeschleunigung $a_{q}(t)$

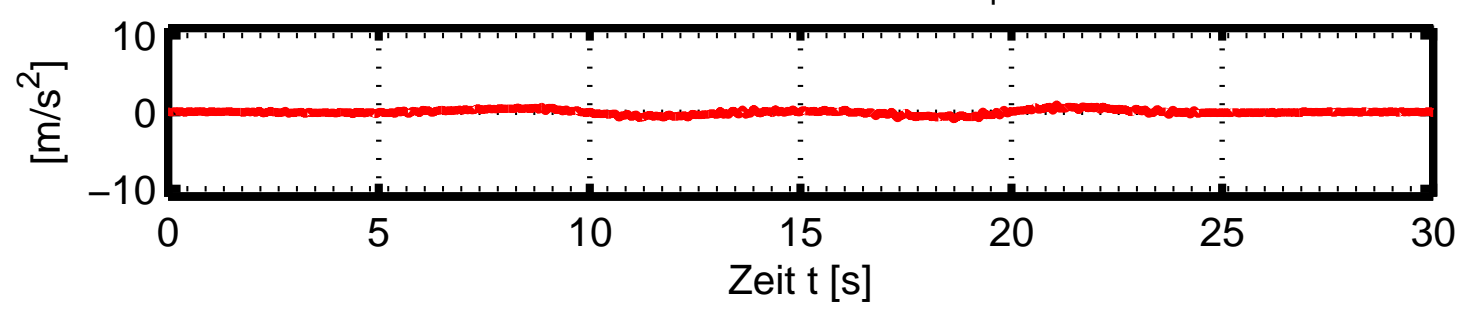

Abbildung 5.10: Messergebnisse eines Doppelspurwechselmanövers (Abbildung 2.2 und 5.6) mit einem Porsche Carrera bei langsamer Fahrt für Position, Geschwindigkeit, Lenkradwinkel, Querabstand, und Querbeschleunigung 

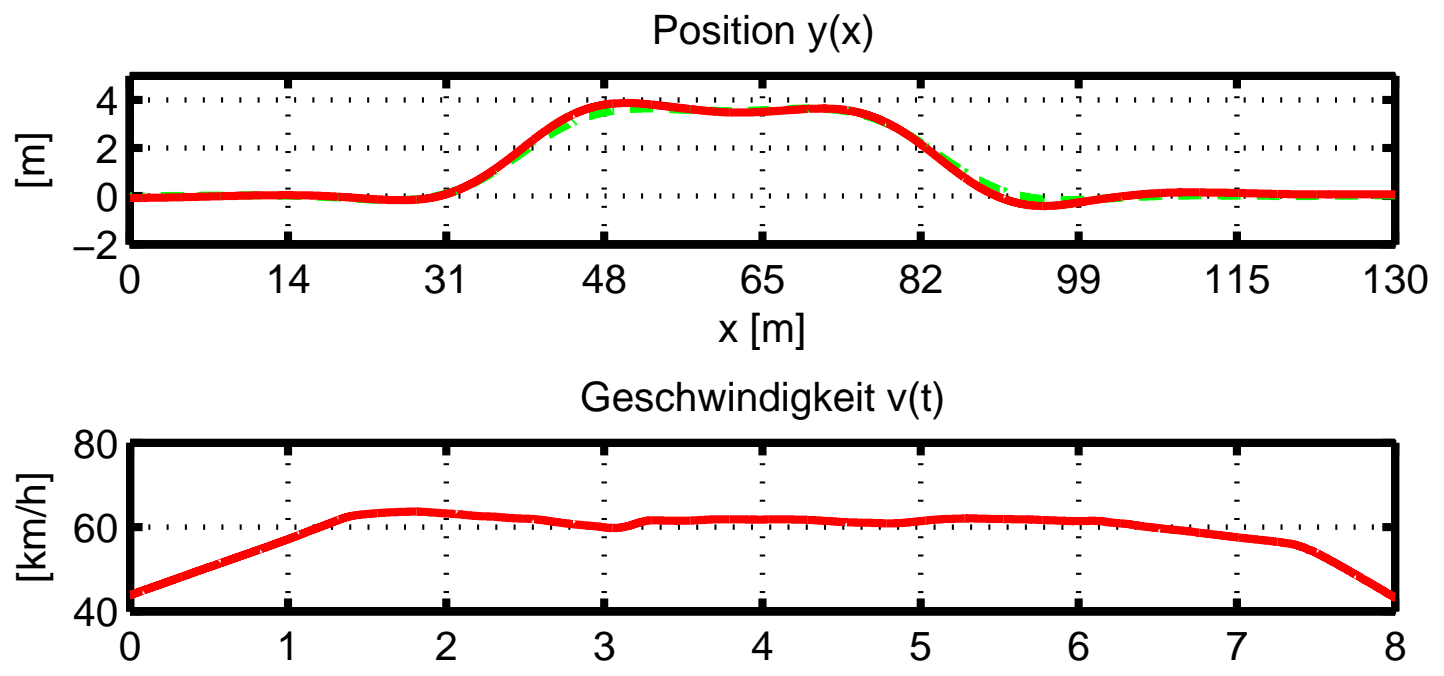

Lenkradwinkel $\delta_{\mathrm{L}}(\mathrm{t})$

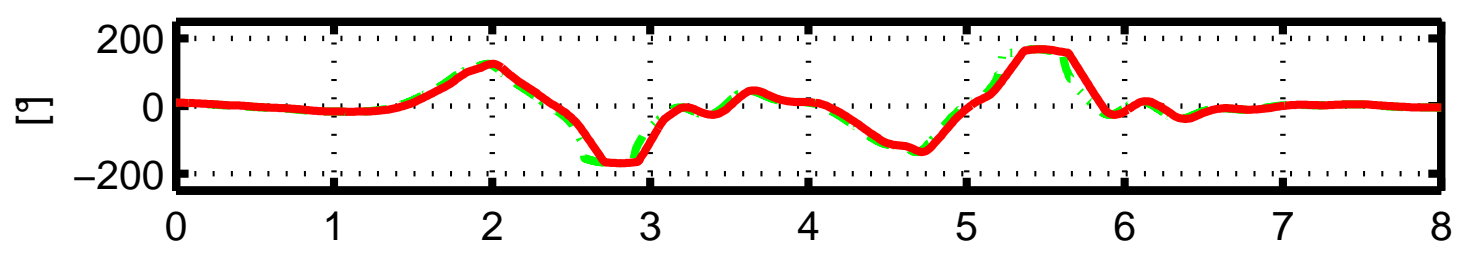

Querabstand $d_{q}(t)$

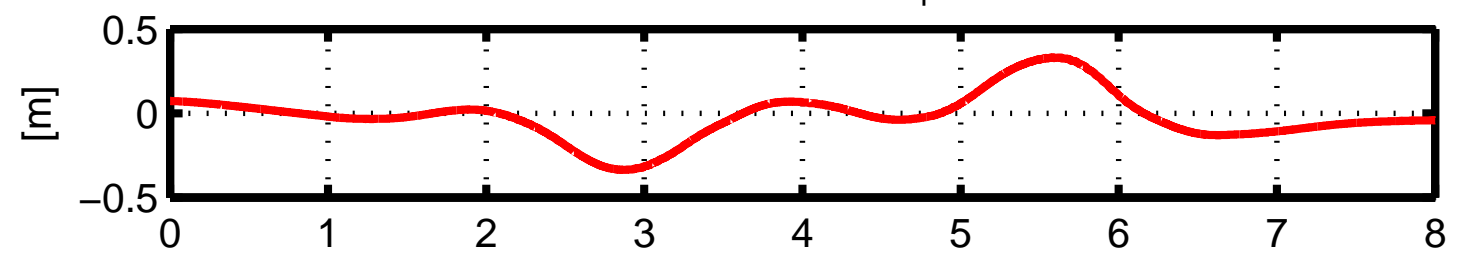

Querbeschleunigung $a_{q}(t)$

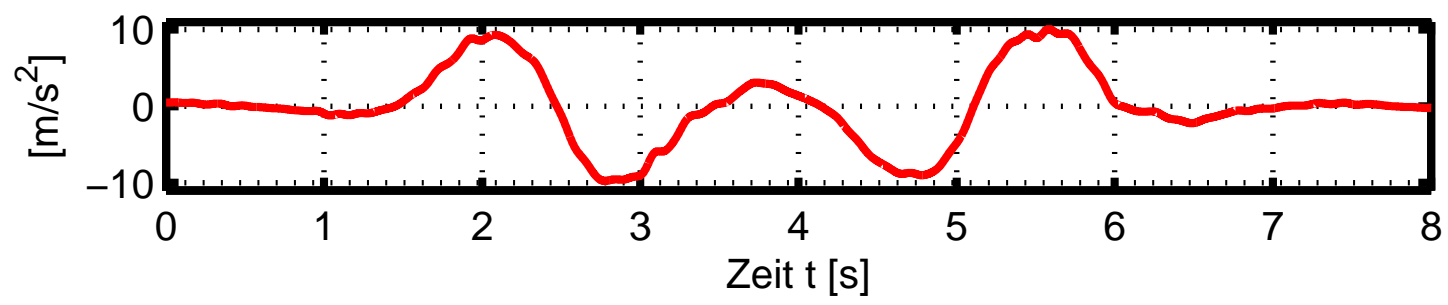

Abbildung 5.11: Messergebnisse eines Doppelspurwechselmanövers (Abbildung 2.2 und 5.6) mit einem Porsche Carrera an der Grenze der Reifenhaftung für Position, Geschwindigkeit, Lenkradwinkel, Querabstand, und Querbeschleunigung 
versucht, die Geschwindigkeit während des Manövers möglichst konstant zu halten. Die Ergebnisse dieses Fahrversuchs sind in Abbildung 5.11 dargestellt.

Das obere Diagramm für die Position zeigt zwei Linien. Eine gestrichelte für die Sollposition und eine durchgezogene für die gemessene Position des Fahrzeugschwerpunktes. In den Kurven sind Abweichungen der gemessenen Position von der Sollposition erkennbar.

Die Geschwindigkeit $v(t)$ liegt wie aus dem zweiten Diagramm ersichtlich nach der Beschleunigungsphase vor dem ersten Richtungswechsel knapp oberhalb von $60 \mathrm{~km} / \mathrm{h}$ und kann trotz des aggressiven Manövers von dem Fahrer annähernd konstant gehalten werden. Bei dieser Geschwindigkeit ist der Folgeregler für schnelle Fahrt auf Basis des dynamischen Fahrzeugmodells aus Abschnitt 4.2.2 aktiv.

Im Diagramm für den Lenkradwinkel $\delta_{L}(t)$ ist die gestrichelte Linie der SollLenkradwinkel und die durchgezogene Linie der gemessene Lenkradwinkel am Lenkmotor. Aufgrund der Stellgrößenbeschränkung auf $1000^{\circ} / \mathrm{s}$ bei einem maximalen Moment von 150Nm kann der Soll-Lenkradwinkel von dem Steuergerät der Lenkmaschine nicht immer eingehalten werden. Im Vergleich zu dem Lenkradwinkel für langsame Fahrt sind hier eine wesentlich höhere Lenkradwinkelaktivität und etwa doppelt so hohe Lenkamplituden zu verzeichnen. In den Zeitabschnitten $t=2.6 \ldots 3.0 \mathrm{~s}$ und $t=5.3 \ldots 5.7 \mathrm{~s}$ sind Knicke im Lenkradwinkel zu erkennen. Sie werden durch die in Abschnitt 4.2.2 beschriebene Begrenzung der inversen Reifenkennlinie in Abbildung 4.7 auf den Schräglaufwinkel für maximale Reifenseitenkraft hervorgerufen. Dies ist ein Indiz für das Erreichen der physikalischen Grenze der Reifenhaftung während des Manövers.

Aufgrund dieser Umstände ist ein schnelles Ansteigen des Querabstandes im vierten Diagramm in den Zeitabschnitten, in denen die Lenkmaschine der Sollvorgabe des Lenkradwinkels nicht folgen kann, zu beobachten. Der maximale Querabstand $d_{q}$ beträgt ca. $35 \mathrm{~cm}$ links und rechts der Sollbahn.

Die Querbeschleunigung $a_{q}$ erreicht das Maximum von ca. $1 \mathrm{~g}\left(9.8 \mathrm{~m} / \mathrm{s}^{2}\right)$. Sie hat trotz der hohen Lenkradwinkelaktivität einen verhältnismäßig glatten und ruckarmen Verlauf.

Im Vergleich zu den Simulationsergebnissen in Abbildung 4.10 zeigt sich im Praxisversuch ein ähnlicher Lenkradwinkel- und Querbeschleunigungsverlauf. Der maximale Querabstand zu der Sollbahn ist in der Simulation jedoch wesentlich kleiner als im Versuch. Die Ursache dafür ist hauptsächlich die Stellgrößenbeschränkung und der in der Praxis mit Unsicherheit behaftete Zustand von Reifen und Fahrbahn. Wird die maximale Querabweichung auf Fahrzeugdimensionen bezogen, entspricht sie ungefähr der Reifenbreite des Fahrzeugs.

Um zu zeigen, daß die in Abbildung 5.11 gewählte Geschwindigkeit von ca. $62 \mathrm{~km} / \mathrm{h}$ an der Grenze der Reifenhaftung für die gegebene Sollbahn liegt, werden in Abbildung 5.12 die Ergebnisse eines Versuchs gezeigt, bei dem die Geschwindigkeit darüber hinaus gesteigert wurde. 

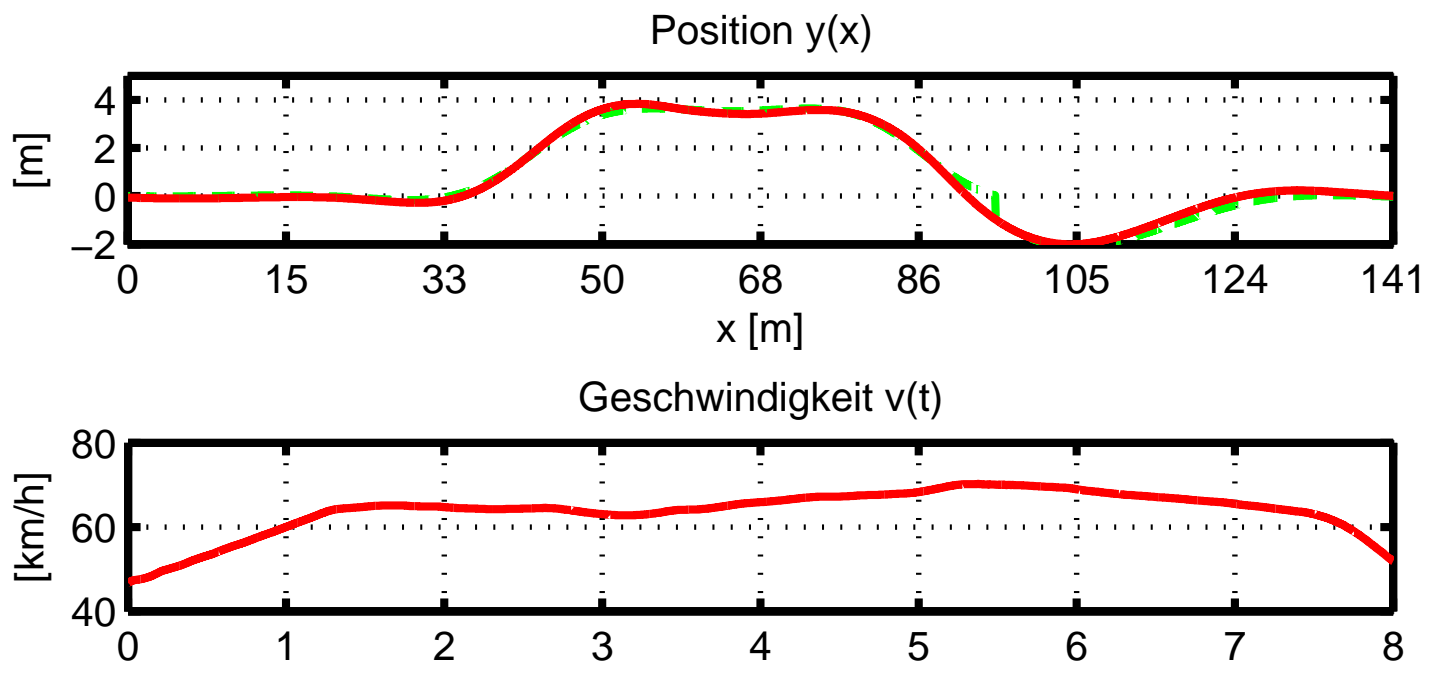

Lenkradwinkel $\delta_{\mathrm{L}}(\mathrm{t})$

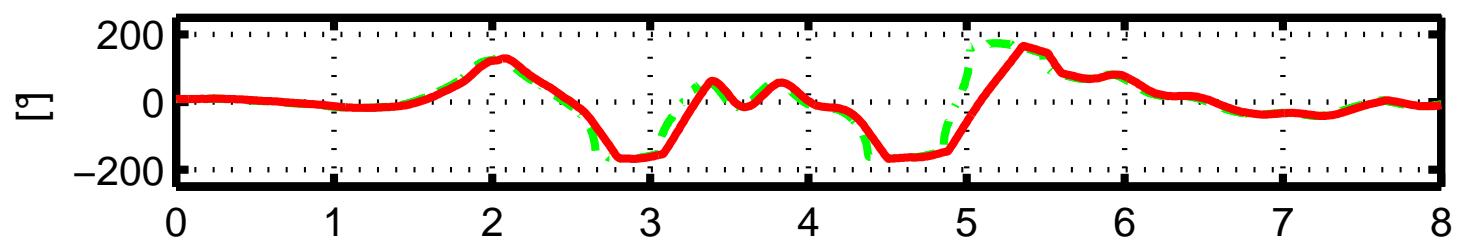

Querabstand $d_{q}(t)$

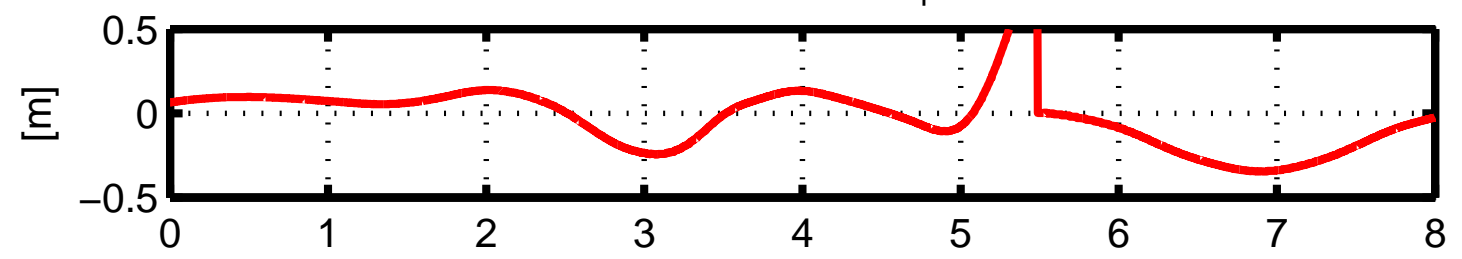

Querbeschleunigung $a_{q}(t)$

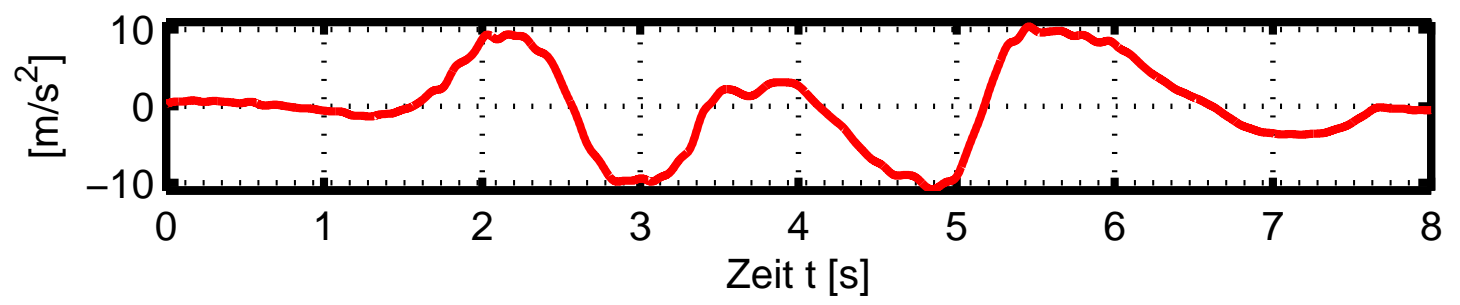

Abbildung 5.12: Messergebnisse eines Doppelspurwechselmanövers (Abbildung 2.2 und 5.6) mit einem Porsche Carrera bei Überschreitung der Reifenhaftgrenze für Position, Geschwindigkeit, Lenkradwinkel, Querabstand, und Querbeschleunigung 
Implementierung und Erprobung der Bahnfolgeregelung

Die Geschwindigkeit, Lenkradwinkel, Querabstand und Querbeschleunigung sind der vorhergehenden Messung bis zum Zeitpunkt $t=3 \mathrm{~s}$ sehr ähnlich. In dem Zeitabschnitt $t=3 \ldots 5 \mathrm{~s}$ wird die Geschwindigkeit um einige $\mathrm{km} / \mathrm{h}$ erhöht. Dies führt ab dem Zeitpunkt $t=5 \mathrm{~s}$ zu einem schnellen Anstieg des Querabstandes wegen Überschreitung der Reifenhaftgrenze. Dies ist in den Diagrammen für Position und Querabstand zu erkennen. Zum Zeitpunkt $t=5.5 \mathrm{~s}$ ist ein Querabstand von $1 \mathrm{~m}$ erreicht. Hier setzt die Neuplanung der Solltrajektorie wie in Abschnitt 2.4 beschrieben ein. Die Querbeschleunigung wird weiter aufrechterhalten, bis der Fahrer die Geschwindigkeit ausreichend reduziert hat und das Fahrzeug die Sollbahn wieder erreicht. Dieser Versuch zeigt, daß die Bahnfolgeregelung das Fahrzeug selbst bei Überschreiten der Reifenhaftgrenze sicher auf die Sollbahn zurückbringen kann.

\subsubsection{Versuchsergebnisse Porsche Cayenne}

Mit Praxistests des entwickelten Bahnfolgereglers für einen sportlichen Geländewagen vom Typ Porsche Cayenne soll gezeigt werden, daß das Regelsystem auf andere Fahrzeugklassen applizierbar ist. Der Geländewagen unterscheidet sich besonders durch einen höher liegenden Schwerpunkt und größere Fahrzeugmasse von dem Sportwagen.

In Abbildung 5.13 sind die Ergebnisse eines mit einem Porsche Cayenne geregelt durchgeführten Doppelspurwechselmanövers dargestellt. Die gewählte Geschwindigkeit während des Manövers bringt das Fahrzeug an die Grenze der Reifenhaftung. In dem Diagramm für die Geschwindigkeit ist zu erkennen, daß die Geschwindigkeit bei Einfahrt in das Manöver knapp unter 60km/h und demzufolge unter der Geschwindigkeit des Porsche Carrera in Abbildung 5.11 liegt. Während des Manövers gelingt es dem Fahrer nicht, die Geschwindigkeit so gut wie bei den Versuchen mit dem Porsche Carrera konstant zu halten. In dem Zeitbereich $t=3 \ldots 5$ s wird die während des ersten Spurwechsels abgefallene Geschwindigkeit durch den Fahrer korrigiert. Das Fahrerassistenzsystem ist über den Taster im Cockpit deaktiviert. Es greift aber dennoch aufgrund der vom System als kritisch beurteilten Fahrsituation während des Manövers bremsend ein. Dies ist in der alltäglichen Fahrpraxis von Nutzen und erleichtert die Regelung durch die geringere Geschwindigkeit bei Enitritt den zweiten Richtungswechsel des Doppelspurwechselmanövers.

Die Verläufe der benötigten Lenkradwinkel $\delta_{L}(t)$ sind für den Porsche Cayenne und den Porsche Carrera sehr ähnlich, wobei die benötigte Lenkradwinkelamplitude für den Geländewagen etwas größer ist. Wie in Abbildung 5.11 weist das Diagramm für den Lenkradwinkel in Abbildung 5.13 Zeitbereiche auf, in denen die Stellgrößenbeschränkung der Lenkmaschine und die Reifenhaftgrenze erreicht wird. Falls die Geschwindigkeit nur etwas höher läge, würde das Fahrzeug wie in Abbildung 5.12 gezeigt von der Sollbahn abkommen.

Für den Porsche Cayenne stellt sich ein ähnlicher Verlauf des Querabstandes $d_{q}(t)$ und 

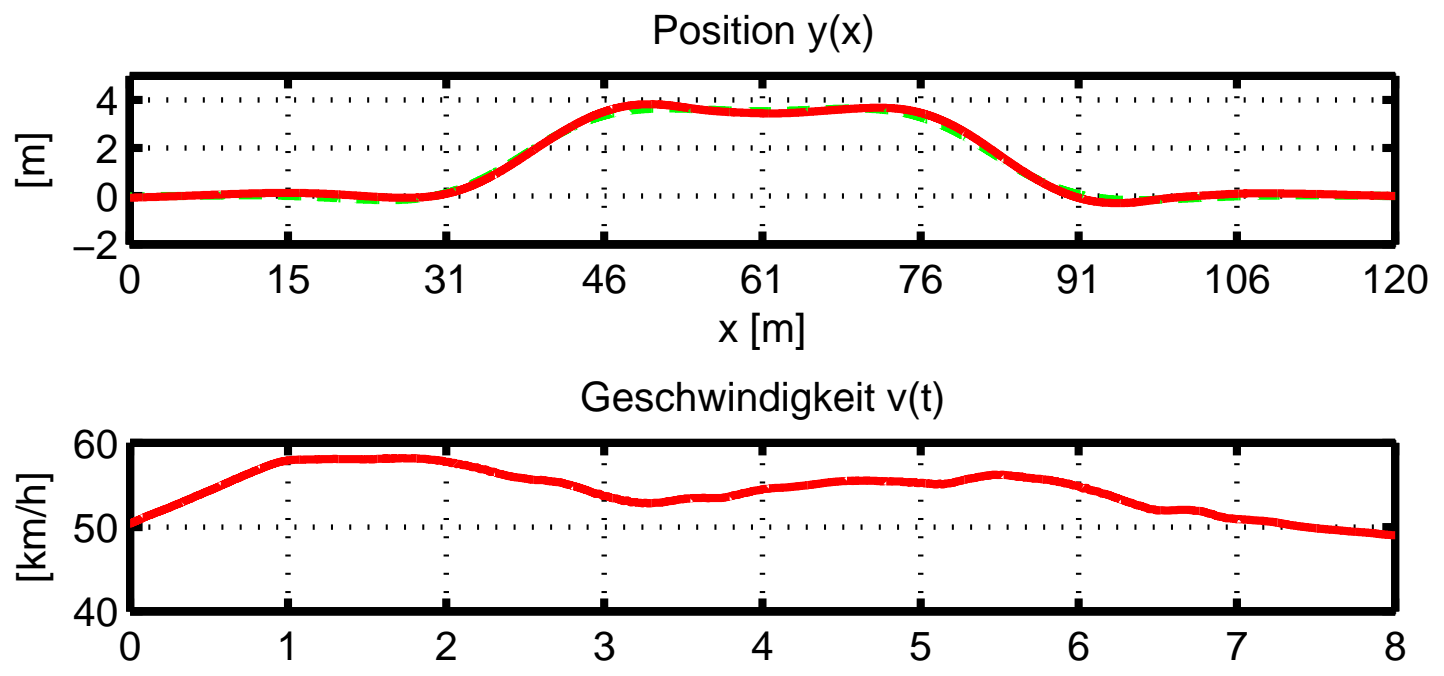

Lenkradwinkel $\delta_{\mathrm{L}}(\mathrm{t})$

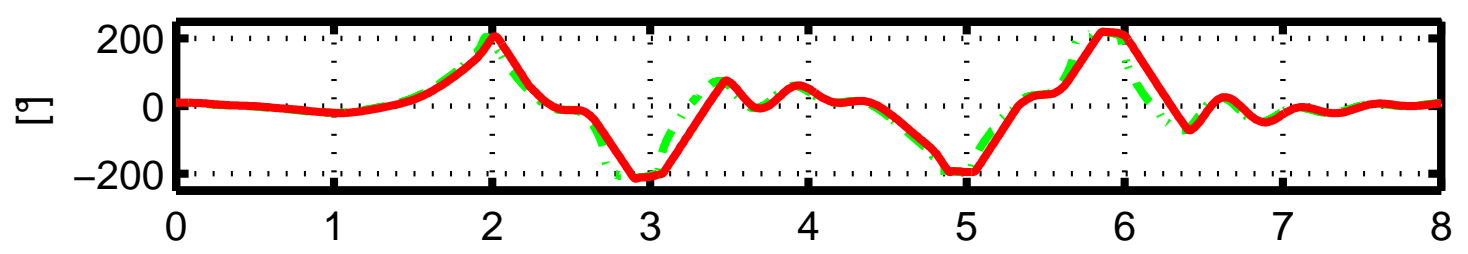

Querabstand $d_{q}(t)$

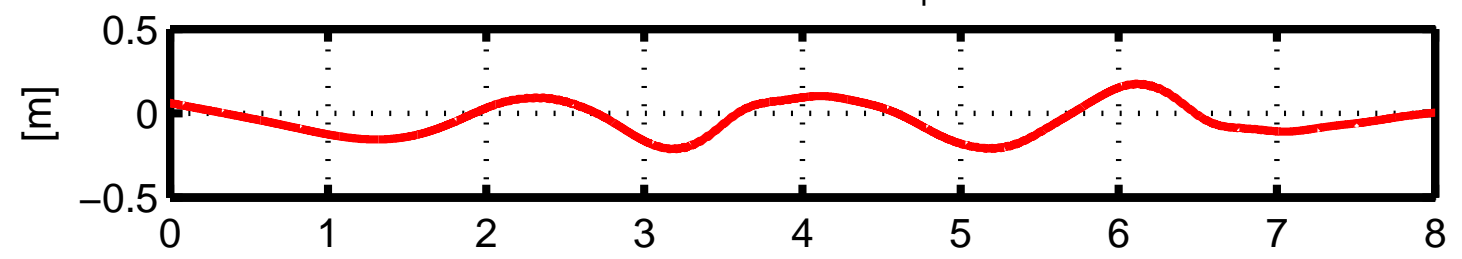

Querbeschleunigung $a_{q}(t)$

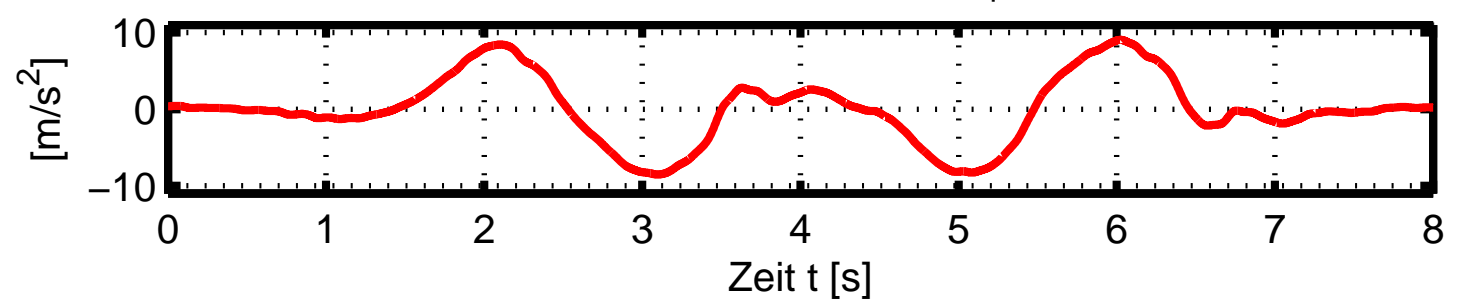

Abbildung 5.13: Messergebnisse eines Doppelspurwechselmanövers (Abbildung 2.2 und 5.6) mit einem Porsche Cayenne an der Grenze der Reifenhaftung für Position, Geschwindigkeit, Lenkradwinkel, Querabstand, und Querbeschleunigung 
Implementierung und Erprobung der Bahnfolgeregelung

der horizontierten Querbeschleunigung $a_{q}(t)$ wie für den Porsche Carrera ein. Dabei ist die maximale Querbeschleunigung für dieselbe Sollbahn etwas geringer, da die gefahrene Geschwindigkeit kleiner ist.

Ergebnisse von Versuchen für langsame Fahrt und das Verhalten bei Überschreitung der Reifenhaftgrenze zeigen keine qualitativen Unterschiede zu den Ergebnissen für den Porsche Carrera.

\subsection{Zusammenfassung}

Mit den vorgestellten Versuchsergebnissen wurde gezeigt, daß sich die Trajektorienplanung, das Navigationssystem und die Bahnfolgeregelung aus den Kapiteln 2 bis 4 erfolgreich in die Systematik des Porsche-Messsystems eingliedern lassen. Das komplette System, ausgenommen die Ansteuerung der Lenkmaschine, wird auf einem Prozessormodul EMD315 so realisiert, daß die zur verfügbare Rechenleistung für eine Regel- und Messfrequenz von $400 \mathrm{~Hz}$ ausreicht.

Das System wird am Beispiel eines Doppelspurwechselmanövers sowohl mit einem Sportwagens des Typs Porsche Carrera als auch mit einem sportlichen Geländewagen des Typs Porsche Cayenne erprobt. Dabei wird die Funktion der Folgeregelung für langsame und schnelle Fahrt aus Abschnitt 4.2 überprüft. Für Fahrten mit Querbeschleunigungen an der physikalischen Grenze der Reifenhaftung werden gemessene Querabweichungen von der Sollbahn kleiner als $35 \mathrm{~cm}$ erreicht. Bei langsamer Fahrt ist die Abweichung kleiner als $5 \mathrm{~cm}$.

Bei Verlassen der Sollbahn durch Überschreiten der Reifenhaftungsgrenze oder durch Stellgrößenbeschränkungen wird das Fahrzeug schnellstmöglich und in kontrollierter Weise zurück auf die Sollbahn geführt. Die Neuplanung der Trajektorie zu Beginn der Fahrt hat sich in den Versuchen als praxistauglich erwiesen. 


\section{Kapitel 6}

\section{Schlußfolgerungen}

Die Erprobung von Fahrzeugen zur Dokumentation des Entwicklungsstandes von Serienfahrzeugen oder Prototypen muß reproduzierbar durchgeführt werden. Nur so sind eine objektive Bewertung und der Vergleich des Fahrverhaltens verschiedener Fahrzeuge möglich. Neben den äußeren Einflüssen wie zum Beispiel Wetter und Fahrbahnbelag hat der Versuchsfahrer einen entscheidenden Einfluß auf die Versuchsergebnisse. Eine erste Stufe für die Reduzierung des Fahrereinflusses bei Fahrversuchen ist der Einsatz einer Lenkmaschine für die Realisierung von gesteuerten Versuchsmanövern mit vordefinierten Lenkradwinkeltrajektorien.

Die in dieser Arbeit realisierte zweite Stufe der Automatisierung von Fahrversuchen ermöglicht die Querregelung des Versuchsfahrzeugs entlang vorgegebener Bahnen. Die modellbasierte Folgeregelung deckt den gesamten Bereich der möglichen Fahrzeugquerbeschleunigung bis an die Grenzen der Reifenhaftung ab. Der Einfluß der Versuchsfahrers wird somit auf den Antriebstrang beschränkt. Erreicht wird die Funktion der Bahnfolgeregelung durch das Zusammenspiel einer hochgenauen integrierten Navigationslösung sowie einer Regelungsstruktur mit zwei Freiheitsgraden auf der Basis geeigneter Fahrzeugmodelle.

Der Beitrag des echtzeitfähigen integrierten Navigationssystems für die Bahnfolgeregelung liegt neben seiner Bedeutung für die spätere Auswertung des Fahrverhaltens des Versuchsfahrzeugs in der nahezu verzögerungsfreien Bereitstellung der Winkellage, Position und Geschwindigkeit der Fahrzeugkarosserie im Raum. Besonders hervorzuheben ist die für die erfolgreiche Realisierung der modellbasierten Vorsteuerung benötigte genaue Messung des Fahrzeugschwimmwinkels als die Differenz von Bewegungsrichtung und Gierwinkel des Fahrzeugs.

Die Trajektorienplanung erzeugt in Echtzeit aus einer vorgegebenen geometrischen Sollbahn eine glatte, mit dem Fahrzeug realisierbare, zeitliche Solltrajektorie für die Vorsteuerung und berechnet die für die lineare PD-Regelung benötigte Querabweichung. 
Da die Fahrzeuggeschwindigkeit durch den Versuchsfahrer bestimmt wird, wird die gemessene Geschwindigkeit in die Bestimmung des Referenzpunktes als dem Fahrzeug nächstgelegene Punkt auf der Sollbahn einbezogen.

Für langsame Fahrten wird ein einfaches kinematisches Fahrzeugmodell für den Entwurf der Vorsteuerung und Regelung verwendet. Das Fahrzeugmodell für die Vorsteuerung bei schneller Fahrt basiert auf dem klassischen Einspurmodell, welches durch an das Versuchsfahrzeug angepaßte Reifenkennlinien erweitert wird. Die Reifenkennlinien für die Vorder- und Hinterräder werden mithilfe der Simulation eines vorhandenen detaillierten und validierten Fahrzeugmodells für stationäre Kurvenfahrt gewonnen. In Verbindung mit der glatten Solltrajektorie wird durch die Reifenkennlinien die Auswirkung des stationären Wankverhaltens des Versuchsfahrzeugs in der Berechnung des Vorsteueranteils berücksichtigt. Dies ermöglicht die Bahnfolge für aggressive Manöver bis an die Grenze der Reifenhaftung.

Die Praxiserprobung der Bahnfolgeregelung zeigt für die zwei Serienfahrzeuge, einen Sportwagen vom Typ Porsche Carrera und einen sportlichen Geländewagen vom Typ Porsche Cayenne, gute Ergebnisse. Für das Doppelspurwechselmanöver mit Querbeschleunigungen bis an die Reifenhaftgrenze beträgt die gemessene maximale Querabweichung weniger als $35 \mathrm{~cm}$. Das Gesamtsystem besteht aus Komponenten der seit Jahren erfolgreich eingesetzten Porsche Messtechnik und ist den im Fahrzeug auftretenden Belastungen gewachsen.

Bei der Vorbereitung von Fahrversuchen mit der entwickelten Bahnfolgeregelung entsteht für den Anwender des Systems kein gerätetechnischer Mehraufwand für die Ausrüstung des Fahrzeugs im Vergleich zu den gesteuerten Versuchen mit vorgegebener Lenkradwinkeltrajektorie. Das integrierte Navigationssystem, dessen Messergebnisse für die Auswertung der Versuche benötigt werden, wird auch für die Regelung verwendet. Das Regelsystem ist zusammen mit dem integrierten Navigationssytstem auf einem einzigen Prozessormodul der Porsche Messtechnik implementiert. Als Aktor für die Regelung wird die für die gesteuerten Manöver verwendete elektrische Lenkmaschine beibehalten.

\section{Ausblick}

Die in dieser Arbeit vorgestelle maschinelle Bahnfolgeregelung eröffnet neue Möglichkeiten für die Definition von Fahrdynamikmanövern. Anstatt eines für den Versuchsfahrer durch Pylonen markierten Korridors kann für jedes Manöver auf dem Prüfgelände eine ortsfeste Sollbahn für die Bahnfolgeregelung definiert und jederzeit reproduzierbar befahren werden. Durch die reproduzierbare Bahnfolge kann neben der erreichbaren Geschwindigkeit beispielsweise die für die Bahnfolge notwendige Lenkradwinkelaktivität als Kriterium für die objektive Beurteilung und einen Vergleich der untersuchten Fahrzeuge herangezogen werden. 
Eine mögliche Weiterentwicklung der realisierten Bahnfolgeregelung besteht in der automatischen Bedienung des Antriebstranges. Dadurch wird eine fahrerlose Fahrt des Versuchsfahrzeugs ermöglicht. Gegenüber der in dieser Arbeit beschriebenen Querregelung mit einer elektrischen Lenkmaschine als einzigem Aktor muss für fahrerlose Fahrt das Problem der Aktorik für die Pedale und das Schaltgetriebe gelöst und insbesondere ein geeignetes Sicherheitskonzept entwickelt werden. Neben Fahrdynamikversuchen könnte die entwickelte Bahnfolgeregelung dann auch für Versuche eingesetzt werden, bei denen ein Versuchsfahrer körperlich stark beansprucht oder sogar gefährdet wird. 


\section{Anhang: Die Dynamikmatrix des Fehlermodells}

Im Folgenden werden die einzelnen Blöcke der Dynamikmatrix F des Fehlermodells (3.13) in Abschnitt 3.2.1 definiert. Dieses Fehlermodell berücksichtigt die Schuler- und CoriolisTerme. Die detaillierte Herleitung ist in [7] gezeigt.

$$
\begin{aligned}
& \mathbf{F}=\left[\begin{array}{lll}
\mathbf{F}_{p p} & \mathbf{F}_{p v} & \mathbf{F}_{p \rho} \\
\mathbf{F}_{v p} & \mathbf{F}_{v v} & \mathbf{F}_{v \rho} \\
\mathbf{F}_{\rho p} & \mathbf{F}_{\rho v} & \mathbf{F}_{\rho \rho}
\end{array}\right] \\
& \mathbf{F}_{p p}=\left[\begin{array}{ccc}
0 & 0 & \frac{-v_{N}}{\left(R_{\lambda}+h\right)^{2}} \\
\frac{v_{E} \sin (\lambda)}{\left(R_{\varphi}+h\right) \cos ^{2}(\lambda)} & 0 & \frac{-v_{E}}{\left(R_{\varphi}+h\right)^{2} \cos (\lambda)} \\
0 & 0 & 0
\end{array}\right] \\
& \mathbf{F}_{p v}=\left[\begin{array}{ccc}
\frac{1}{R_{\lambda}+h} & 0 & 0 \\
0 & \frac{1}{\left(R_{\varphi}+h\right) \cos (\lambda)} & 0 \\
0 & 0 & -1
\end{array}\right] \\
& \mathbf{F}_{p \rho}=\mathbf{0} \\
& \mathbf{F}_{v p}=\left[\begin{array}{ccc}
-\left[\frac{v_{e}^{2}}{\left(R_{\varphi}+h\right) \cos ^{2}(\lambda)}+2 \omega_{i e} v_{e} \cos (\lambda)\right] & 0 & \frac{v_{E}^{2} \tan (\lambda)}{\left(R_{\varphi}+h\right)^{2}}+\frac{-v_{N} v_{D}}{\left(R_{\lambda}+h\right)^{2}} \\
\frac{v_{E} v_{N}}{\left(R_{\varphi}+h\right) \cos ^{2}(\lambda)}+2 \omega_{i e}\left[v_{N} \cos (\lambda)-v_{D} \sin (\lambda)\right] & 0 & -v_{E}\left[\frac{v_{N} \tan (\lambda)+v_{D}}{\left(R_{\varphi}+h\right)^{2}}\right] \\
v_{E} 2 \omega_{i e} \sin (\lambda) & 0 & \frac{v_{E}^{2}}{\left(R_{\varphi}+h\right)^{2}}+\frac{v_{N}^{2}}{\left(R_{\lambda}+h\right)^{2}}-2 \frac{g}{R}
\end{array}\right]
\end{aligned}
$$


$\mathbf{F}_{v v}=\left[\begin{array}{ccc}\frac{v_{D}}{R_{\lambda}+h} & -\left[\frac{2 v_{E}}{\left(R_{\varphi}+h\right) \cos (\lambda)}+2 \omega_{i e}\right] \sin (\lambda) & \frac{v_{N}}{R_{\lambda}+h} \\ {\left[\frac{v_{E} \tan (\lambda)}{R_{\varphi}+h}+2 \omega_{i e} \sin (\lambda)\right]} & \frac{v_{N} \tan (\lambda)}{R_{\varphi}+h}+\frac{v_{D}}{R_{\varphi}+h} & \frac{v_{E}}{R_{\varphi}+h}+2 \omega_{i e} \cos (\lambda) \\ \frac{-2 v_{N}}{R_{\lambda}+h} & -\left[\frac{2 v_{E}}{R_{\varphi}+h}+2 \omega_{i e} \cos (\lambda)\right] & 0\end{array}\right]$

$\mathbf{F}_{v \rho}=\left[\begin{array}{ccc}0 & f_{D} & -f_{E} \\ -f_{D} & 0 & f_{N} \\ f_{E} & -f_{N} & 0\end{array}\right]$

$\mathbf{F}_{\rho p}=\left[\begin{array}{ccc}\omega_{i e} \sin (\lambda) & 0 & \frac{v_{E}}{\left(R_{\varphi}+h\right)^{2}} \\ 0 & 0 & \frac{-v_{N}}{\left(R_{\lambda}+h\right)^{2}} \\ \omega_{i e} \cos (\lambda)+\frac{v_{E}}{\left(R_{\varphi}+h\right) \cos ^{2}(\lambda)} & 0 & \frac{-v_{E} \tan (\lambda)}{\left(R_{\varphi}+h\right)^{2}}\end{array}\right]$

$\mathbf{F}_{\rho v}=\left[\begin{array}{ccc}0 & \frac{-1}{R_{\varphi}+h} & 0 \\ \frac{1}{R_{\lambda}+h} & 0 & 0 \\ 0 & \frac{\tan (\lambda)}{R_{\varphi}+h} & 0\end{array}\right]$

$\mathbf{F}_{\rho \rho}=\left[\begin{array}{ccc}0 & \omega_{D} & -\omega_{E} \\ -\omega_{D} & 0 & \omega_{N} \\ \omega_{E} & -\omega_{N} & 0\end{array}\right]$ 


\section{Literaturverzeichnis}

[1] Bronstein, Semendjajew, Musiol und Mühlig: Taschenbuch der Mathematik. 3. Ausgabe. Verlag Harri Deutsch, 1997.

[2] Cohen, C. E.: Attitude Determination, volume 164 of Progress in Astronautics and Aeronautics, chapter 19, pages 519-538. American Institute of Aeronautics and Astronautics, Inc., 1995.

[3] Dambeck, J.H.: Diagnose und Therapie geodätischer Trägheitsnavigationssysteme. Dissertation Universität Stuttgart, 1998.

[4] DIN 70000: Fahrzeugdynamik und Fahrverhalten. Deutsches Institut für Normung e.V., 1994.

[5] DIN ISO 4138: Straßenfahrzeuge - Stationäre Kreisfahrt. Deutsches Institut für Normung e.V., 1984.

[6] DIN ISO 7401: Testverfahren für querdynamisches Übertragungsverhalten. Deutsches Institut für Normung e.V., 1989.

[7] Farrell, J. A. and M. Barth: The Global Positioning System 8 Inertial Navigation. McGraw-Hill, 1998.

[8] Föllinger, Otтo: Regelungstechnik. Hüthig Buch Verlag, 1994.

[9] Götting, H.-H.: Automatisiertes Fahren mit Nutzfahrzeugen. Firmenschrift der Götting KG, 2001.

[10] Hagenmeyer, V. und M. Zeitz: Flachheitsbasierter Entwurf von linearen und nichtlinearen Vorsteuerungen. Automatisierungstechnik, 52:3-12, 2004.

[11] Herzer, B.: Entwicklung eines GPS-gestützten inertialen Navigationssystems. Praktikumsbericht. Universität Stuttgart, April 2004.

[12] Hofmann-Wellenhof, B., H. Lichtenegger, and J. Collins: Global Positioning System, Theory and Practice. Springer, 5th edition, 2001. 
[13] ISO 3888-1:1999: Passenger cars - Test track for a severe lane-change manoeuvre. International Organization for Standardization, 1999.

[14] KeHL, S.: Stützung eines inertialen Navigationssystems mittels GPS. Diplomarbeit, Universität Stuttgart, Institut für Systemdynamik und Regelungstechnik, 2003.

[15] Kehl, S., W.-D. PÖLsLer und M. Zeitz: Entwurf und Erprobung der Querregelung eines Versuchsfahrzeugs entlang vorgegebener Bahnen. In: 3. Fachtagung Steuerung und Regelung von Fahrzeugen und Motoren - AUTOREG 2006. VDI-Berichte Nr. 1931, VDI Verlag GmbH, 99-108, 2006.

[16] Kehl, S., W.-D. Pölsler und M. Zeitz: Querregelung eines PKWs entlang vorgegebener Bahnen. In: GMA Kongress 2005. VDI-Berichte Nr. 1883, VDI Verlag GmbH, 835-842, 2005.

[17] Kehl, S., W.-D. PÖLsler, and M. ZeItz: Vehicle path-following with a gpsaided inertial navigation system. In Meurer, T., K. Graichen, and E. D. Gilles (editors): Control and Observer Design for Nonlinear Finite and Infinite Dimensional Systems. LNCIS Springer-Verlag, 285-300, 2005.

[18] Kehl, S., W.-D. PÖLsLer und M. ZeItZ: Querregelung eines Versuchsfahrzeugs entlang vorgegebener Bahnen. at-Automatisierungstechnik, Oldenbourg Wissenschaftsverlag, 55:306-313, 2007.

[19] LÖWIS, J. VON: Flachheitsbasierte Trajektorienfolgeregelung elektromechanischer Systeme. Shaker-Verlag, Aachen, 2002.

[20] M. S. Grewal, L. R. Weill and A. P. Andrews: Golbal Positioning Systems, Inertial Navigation and integration. John Wiley \& Sons Inc., 2000.

[21] Maybeck, P. S.: Stochastic Models, Estimation and Control, volume 1. Academic Press, 1979.

[22] Milliken, W.F. and D.L. Milliken: Race Car Vehicle Dynamics. SAE International, 1995.

[23] Neukum, A., H.-P. Krüger und J. Schuller: Der Fahrer als Messinstrument für fahrdynamische Eigenschaften? In: Der Fahrer im 21. Jahrhundert. VDI-Berichte Nr. 1613, 2001.

[24] Nieuwstadt, M.J. van and R.M. Murray: Real time trajectory generation for differentially flat systems. International Journal on Robust and Nonlinear Control, 8:995-1020, 1998.

[25] Peugeot FR: Besuch im Forschungs-und Entwicklungszentrum von Peugeot. Autotechnik, 3-6, 1981. 
[26] Porsche AG, Dr. Ing. H.C. F.: 911 Kurz und komplett; Modelljahr 2004.

[27] Porsche AG, Dr. Ing. H.C. F.: Cayenne Kurz und komplett; Modelljahr 2004.

[28] Riekert, P. und T. E. Schunck: Zur Fahrmechanik des gummibereiften Kraftfahrzeugs. Ingenieur-Archiv, XI:210-224, 1940.

[29] Shin, Eun-Hwan: Accuracy Improvement of Low Cost GPS/INS for Land Applications. 2001.

[30] Shladover, S. E.: Review of the State of Development of Advanced Vehicle Control Systems (AVCS). Vehicle System Dynamics, 24:551-595, 1995.

[31] Simon, D.: Data Smoothing and Interpolation Using Eighth Order Algebraic Splines. Cleveland State University, Department of Electrical and Computer Engineering, 2003.

[32] SöHnitz, I.: Querregelung eines autonomen Straßenfahrzeugs. Dissertation, Technische Universität Braunschweig, 2001.

[33] Stiller, C., S. Kammel, T. Dang, C. Duchow und B. Hummel: Das Autonome Landfahrzeug ION im Grand Challenge. In: Steuerung und Regelung von Fahrzeugen und Motoren - AUTOREG 2006. VDI Berichte Nr. 1931, 75-88, 2006.

[34] Sukkarieh, S., E.M. Nebot, and H.F. Durrant-Whyte: Achiving Integrity in an INS/GPS Navigation Loop for Autonomous Land Vehicle Applications. IEEE, 1998.

[35] Titterton, D.H. and J.L. Weston: Strapdown Inertial Navigation Technology. Peter Peregrinus, 1997.

[36] WagneR, J.: Zur Verallgemeinerung integrierter Navigationssysteme auf räumlich verteilte Sensoren und flexible Fahrzeugstrukturen. Fortschritt-Berichte Reihe $8 \mathrm{Nr}$. 1008. VDI, 2003.

[37] WagneR, N.: Fahrerassistenzsysteme - Konzepte und Anforderungen. In: Automatisierung- und Assistenzsysteme für Transportmittel. Gesamtzentrum für Verkehr Braunschweig e.V., 2004.

[38] Zeitz, M., K. Graichen und T. Meurer: Vorsteuerung mit Trajektorienplanung als Basis einer Folgeregelung. In: GMA Kongress 2005. VDI-Berichte Nr. 1883, VDI/VDE, 795-806, 2005.

[39] ZogG, J.-M.: GPS Grundlagen. Firmenschrift der $\mu$-blox AG, 2003. 